\title{
RESIDUE MIRROR SYMMETRY FOR GRASSMANNIANS
}

\author{
BUMSIG KIM, JEONGSEOK OH, KAZUSHI UEDA, AND YUTAKA YOSHIDA
}

\begin{abstract}
Motivated by recent works on localizations in A-twisted gauged linear sigma models, we discuss a generalization of toric residue mirror symmetry to complete intersections in Grassmannians.
\end{abstract}

\section{Contents}

1. Introduction

2. Correlation functions of A-twisted gauged linear sigma models

3. Quasimap spaces for projective spaces

4. Projective complete intersections

5. Concave bundles on projective spaces

6. Classical mirror symmetry for toric hypersurfaces

7. Quasimap correlation functions for anti-canonical hypersurfaces in toric varieties

8. Toric residue mirror symmetry

9. Martin's formula

10. Quasimap spaces for GIT quotients

11. Quasimap spaces for Grassmannians

12. Residue mirror symmetry for Grassmannians

13. Bethe/gauge correspondence

14. Quasimaps and instantons

15. Quasimaps and monopoles

16. Quasimaps and vortices

References

\section{INTRODUCTION}

A-twisted gauged linear sigma models are 2-dimensional topological field theories introduced by Witten [Wit93. An A-twisted gauged linear sigma model is specified by a reductive algebraic group $G$ (or its compact real form) called the gauge group, an affine space $W$ with a linear action of $G \times \mathbb{G}_{\mathrm{m}}$ called the matter, and an element $\xi$ of the dual $\mathfrak{z}^{*}$ of the center of the Lie algebra of $G$ called the Fayet-Iliopoulos parameter. The weights of the $\mathbb{G}_{\mathrm{m}}$-actions are called $R$-charges. One can also introduce a superpotential in the theory, which is a $G$-invariant function on $W$ of R-charge 2. The correlation functions, which are quantities of primary interest, do not depend on the potential.

An A-twisted gauged linear sigma model with a suitable Fayet-Iliopoulos parameter is expected to be equivalent to the topological sigma model whose target is the classical vacuum subspace of the symplectic reduction $W / /{ }_{\xi} G$. This comes from a stronger expectation that the low-energy limit of a gauged linear sigma model should give the non-linear sigma model whose target is the symplectic reduction $W / / \xi G$. 
A prototypical example is the case $G=\mathbb{G}_{\mathrm{m}}$ and $W=\mathbb{A}^{6}$, with the action

$$
G \times \mathbb{G}_{\mathrm{m}} \ni(\alpha, \beta):\left(z_{1}, \ldots, z_{5}, P\right) \mapsto\left(\alpha z_{1}, \ldots, \alpha z_{5}, \alpha^{-5} \beta^{2} P\right)
$$

and a potential $P f$, which is the product of the variable $P$ and a homogeneous polynomial $f$ in $z_{1}, \ldots, z_{5}$ of degree 5 . The symplectic reduction $W / / \xi G$ for the positive $\xi$ gives the total space of the bundle $\mathcal{O}_{\mathbb{P}^{4}}(-5)$. The R-charge of the $P$-field indicates that the target space should be considered not as a manifold but as a supermanifold, where the parity of the fiber is odd.

One candidate for a mathematical theory of A-twisted gauged linear sigma models is symplectic vortex invariants [CGS00, CGMiRS02, MiR03, MiRT09, GW13, Zil14] and their generalizations incorporating potentials [TX, FJR18. Another candidate is quasimap theory, which is an intersection theory on moduli spaces of maps to the quotient stacks $[W / G]$. A review of the latter theory, with historical remarks and extensive references, can be found in [CFK]. These two approaches should be related by HitchinKobayashi correspondence for vortices [Bra91, MiR00, VW16].

When the gauge group is abelian, quasimap theory as a mathematical theory of Atwisted gauged linear sigma models goes back to [MP95]. The relation with the Yukawa coupling of the mirror is formulated as toric residue mirror conjecture in [BM02, BM03] and proved in SV04, Bor05, Kar05, SV06.

Quasimap theory in the special case of projective hypersurfaces is also studied in the insightful paper Giv95a], where a heuristic relation with semi-infinite homologies of loop spaces is discussed. This eventually leads to Givental's proof Giv96] of classical mirror symmetry [CdlOGP91] for the quintic 3-fold. This has been extended to toric complete intersections in Giv98.

The correlation functions of A-twisted gauged linear sigma models in the cases when gauge groups are not necessarily abelian are computed in [BZ15, CCP15] using supersymmetric localization of path integrals. The result is given in terms of Jeffrey-Kirwan residues, and reproduces the results of [MP95] in abelian cases.

The aim of this paper is twofold. One is to give an expository account of quasimap theory and its relation to other subjects such as instantons and integrable systems. The other is to formulate Conjecture 10.10, which states that the correlation function defined in (2.2.4) in terms of residues coincides with the generating function of quasimap invariants defined in (10.9.3), and prove it for Grassmannians in Section 12, This can be considered as a generalization of toric residue mirror symmetry to Grassmannians. We also show in Section 8 that a slightly weakened version of toric residue mirror conjecture follows from Givental's mirror theorem. Nothing else in this paper is new.

This paper is organized as follows: In Section 2, we recall the description of correlation functions of A-twisted gauged linear sigma models given in [BZ15, CCP15]. In Section 3, we recall the definition of the quasimap spaces $\mathbf{Q}\left(\mathbb{P}^{n-1} ; d\right)$. They are compactifications of the spaces of holomorphic maps of degrees $d$ from $\mathbb{P}^{1}$ to $\mathbb{P}^{n-1}$, and play an essential role in Givental's homological geometry [Giv95a, Giv95b]. In Section 4, we recall toric residue mirror symmetry for Calabi-Yau complete intersections in projective spaces. In Section 5, we discuss quasimap invariants of concave bundles. In Section 6, we recall classical mirror symmetry for toric hypersurfaces proved in Giv98. The exposition in Section 6 follows [ri11] closely. In Section 7, we briefly recall the definition of quasimap spaces for toric varieties due to [MP95. In Section 8, we show that a slightly weakened version of toric residue mirror conjecture for CY hypersurfaces follows from classical mirror symmetry. In Section 9, we recall a theorem of Martin which relates integration on a symplectic quotient by a compact Lie group to that on the quotient by a maximal torus. In Section 10, 
we recall the definition of quasimap spaces to GIT quotients, which are called quasimap graph spaces in CFK14. The quasimap spaces come with the universal $G$-bundle and the canonical virtual fundamental classes, which allow us to define numerical invariants. We formulate Conjecture 10.10, which states that correlation functions of A-twisted gauged linear sigma models given in (2.2.4) are generating functions of quasimap invariants. There is a natural $\mathbb{G}_{\mathrm{m}}$-action on the quasimap graph space coming from the $\mathbb{G}_{\mathrm{m}}$-action on the domain curve. There is a distinguished connected component of the fixed locus of this action, which is used to define the I-function. In Section 11, the quasimap spaces and the $I$-functions for Grassmannians are recalled from BCFK05. In Section 12, we prove Conjecture 10.10 for Grassmannians. For this purpose, we introduce abelianized quasimap spaces for Grassmannians, which allows us to relate quasimap invariants for Grassmannians with correlations function in (2.2.4). In Section 13, we discuss the relation between gauged linear sigma models and Bethe ansatz following [NS09]. In Sections 14, 15. and 16, we recall the relations of quasimaps with instantons, monopoles, and vortices respectively.

Acknowledgements: We thank Ionut Ciocan-Fontanine, Hiroshi Iritani, and Makoto Miura for valuable discussions. We also thank the anonymous referees for suggesting several improvements, and Korea Institute for Advanced Study for financial support and excellent research environment. K. U. is supported by JSPS KAKENHI Grant Numbers 24740043, $15 \mathrm{KT} 0105,16 \mathrm{~K} 13743$, and $16 \mathrm{H} 03930$. B. K. is supported by the KIAS individual grant MG016403.

\section{Correlation functions of A-Twisted gauged linear sigma models}

2.1. Let $G$ be a reductive algebraic group of rank $r$, and $W$ be a representation of $G \times \mathbb{G}_{\mathrm{m}}$. The center of $G$ and its Lie algebra will be denoted by $Z(G)$ and $\mathfrak{z}$. Fix a maximal torus $T$ of $G$, and let $\mathfrak{t}$ be its Lie algebra. The set of roots, its subset of positive roots, and the Weyl group will be denoted by $\Delta, \Delta_{+}$, and $\mathscr{W}:=N(T) / T$. Let $W=\bigoplus_{i=1}^{N} W_{i}$ be the weight space decomposition of $W$ with respect to the action of $T \times \mathbb{G}_{\mathrm{m}}$. The weight of $W_{i}$ will be denoted by $\left(\rho_{i}, r_{i}\right) \in \mathfrak{t}^{\vee} \oplus \mathbb{Z}$, and $r_{i}$ will be called the $R$-charge. If $W$ admits an action of another torus $H$ commuting with the action of $G \times \mathbb{G}_{\mathrm{m}}$, then one can introduce the twisted mass $\lambda \in \mathfrak{h}$ in the theory, which corresponds to the equivariant parameter for the $H$ action. The $T \times \mathbb{G}_{\mathrm{m}} \times H$-weight of $W_{i}$ will be denoted by $\left(\rho_{i}, r_{i}, \nu_{i}\right) \in \mathfrak{t}^{\vee} \oplus \mathbb{Z} \oplus \mathfrak{h}^{\vee}$. We also introduce the complexified Fayet-Illiopoulos parameter $t^{\prime} \in \mathfrak{z}^{\vee} \otimes_{\mathbb{R}} \mathbb{C}$, which corresponds to the complexified Kähler form of the symplectic quotient. Here, we save the unprimed symbol $t$ for the indeterminate in the generating function of quasimap invariants (see (3.4.1), (3.11.1), (4.2.4) and (12.7.4)).

For $d \in \mathfrak{t}$ and $t^{\prime} \in \mathfrak{z}^{\vee}$, the composition of the surjection $\mathfrak{t}^{\vee} \rightarrow \mathfrak{z}^{\vee}$ dual to the inclusion $\mathfrak{z} \hookrightarrow \mathfrak{t}$ and the evaluation $\mathfrak{t}^{\vee} \times \mathfrak{t} \rightarrow \mathbb{C}$ will be denoted by $t^{\prime} \cdot d$ or $t^{\prime}(d)$.

\subsection{For $d \in \mathfrak{t}$ and $x \in \mathfrak{t}$, let}

$$
Z_{d}(x):=Z_{d}^{\mathrm{vec}}(x) Z_{d}^{\mathrm{mat}}(x)
$$

be the product of

$$
Z_{d}^{\mathrm{vec}}(x):=\prod_{\alpha \in \Delta_{+}}(-1)^{\alpha(d)+1} \alpha^{2}(x)
$$


and

$$
Z_{d}^{\mathrm{mat}}(x):=\prod_{i=1}^{N}\left(\rho_{i}(x)+\nu_{i}(\lambda)\right)^{r_{i}-\rho_{i}(d)-1} .
$$

Here the superscripts 'vec' and 'mat' stands for the vector multiplet and the matter chiral multiplet respectively. According to [BZ15, CCP15], the correlation function of a $\mathscr{W}$-invariant polynomial $P(x) \in \mathbb{C}[\mathfrak{t}]^{\mathscr{W}}$ on a 2 -sphere is given, up to sign introduced by hand, by

$$
\langle P(x)\rangle_{\mathrm{GLSM}}=\frac{1}{|\mathscr{W}|} \sum_{d \in \mathrm{P} \vee} e^{t^{\prime} \cdot d} \operatorname{JK}_{\mathfrak{c}}\left(Z_{d}(x) P(x)\right) .
$$

Here $\mathrm{P}^{\vee}$ is the coweight lattice of $G$ and $\mathrm{JK}_{\mathfrak{c}}$ is the Jeffrey-Kirwan residue defined in [SV04, Section 2] (cf. also [BV99]). The cone $\mathfrak{c} \subset \mathfrak{z}^{\vee}$ is the ample cone of the GIT quotient determined by the Fayet-Iliopoulos parameter $\eta$.

2.3. One can introduce a variable $\mathbf{z}$ associated with the background value of an auxiliary gauge field in the gravity multiplet. This corresponds to the equivariant parameter for the $\mathbb{G}_{\mathrm{m}}$-action on the domain curve. This turns (2.2.1) into the product

$$
Z_{d}(x ; \mathbf{z}):=Z_{d}^{\mathrm{vec}}(x ; \mathbf{z}) Z_{d}^{\mathrm{mat}}(x ; \mathbf{z})
$$

of

$$
Z_{d}^{\mathrm{vec}}(x ; \mathbf{z}):=\prod_{\alpha \in \Delta_{+}}(-1)^{\alpha(d)+1} \alpha(x) \alpha(x+d \mathbf{z})
$$

and

$$
Z_{d}^{\mathrm{mat}}(x ; \mathrm{z}):=\prod_{i=1}^{N} \frac{\prod_{l=-\infty}^{-1}\left(\rho_{i}(x)+\nu_{i}(\lambda)-\left(l+\frac{r_{i}}{2}\right) \mathrm{z}\right)}{\prod_{l=-\infty}^{\rho_{i}(d)-r_{i}}\left(\rho_{i}(x)+\nu_{i}(\lambda)-\left(l+\frac{r_{i}}{2}\right) \mathrm{z}\right)},
$$

and the correlation function of $P(x) \in \mathbb{C}[\mathfrak{t}]^{\mathscr{W}}$ is given by

$$
\langle P(x)\rangle_{\mathrm{GLSM}}^{H \times \mathbb{G}_{\mathrm{m}}}=\frac{1}{|\mathscr{W}|} \sum_{d \in \mathrm{P}^{\vee}} e^{t^{\prime} \cdot d} \operatorname{JK}_{\mathfrak{c}}\left(Z_{d}(x ; \mathrm{z}) P(x)\right) .
$$

2.4. Another quantity of interest is the effective twisted superpotential on the Coulomb branch, or the effective potential for short. It is defined as the sum

$$
W_{\text {eff }}\left(x ; t^{\prime}\right):=W_{\mathrm{FI}}\left(x ; t^{\prime}\right)+W_{\mathrm{vec}}(x)+W_{\text {mat }}(x)
$$

of the Fayet-Illiopoulos term

$$
W_{\mathrm{FI}}\left(x ; t^{\prime}\right):=t^{\prime} \cdot x
$$

the vector multiplet term

$$
W_{\text {vec }}(x):=-\pi \sqrt{-1} \sum_{\alpha \in \Delta^{+}} \alpha(x),
$$

and the matter term

$$
W_{\text {mat }}(x):=-\sum_{i=1}^{N}\left(\rho_{i}(x)+\nu_{i}(\lambda)\right)\left(\log \left(\rho_{i}(x)+\nu_{i}(\lambda)\right)-1\right) .
$$




\section{QuASIMAP SPACES FOR PROJECTIVE SPACES}

3.1. A holomorphic map $u: \mathbb{P}^{1} \rightarrow \mathbb{P}^{n-1}$ of degree $d$ is given by a collection $\left(u_{i}\left(z_{1}, z_{2}\right)\right)_{i=1}^{n}$ of $n$ homogeneous polynomials of degree $d$ satisfying the following condition:

(3.1.1) There exists no $\left(z_{1}, z_{2}\right) \in \mathbb{A}^{2} \backslash\{0\}$ such that $u\left(z_{1}, z_{2}\right)=0 \in \mathbb{A}^{n}$.

Two collections $\left(u_{i}\left(z_{1}, z_{2}\right)\right)_{i=1}^{n}$ and $\left(u_{i}^{\prime}\left(z_{1}, z_{2}\right)\right)_{i=1}^{n}$ define the same map if and only if there exists $\alpha \in \mathbb{G}_{\mathrm{m}}$ such that $u_{i}\left(z_{1}, z_{2}\right)=\alpha u_{i}^{\prime}\left(z_{1}, z_{2}\right)$ for all $i \in\{1, \ldots, n\}$. It follows that the space

$$
\mathcal{M}\left(\mathbb{P}^{n-1} ; d\right):=\left\{u: \mathbb{P}^{1} \rightarrow \mathbb{P}^{n-1} \mid \operatorname{deg} u=d\right\}
$$

of holomorphic maps of degree $d$ from $\mathbb{P}^{1}$ to $\mathbb{P}^{n-1}$ can be compactified to the projective space of dimension $n(d+1)-1$, whose homogeneous coordinate is given by the coefficients $\left(a_{i j}\right)_{i, j}$ of the collection $\left(u_{i}\left(z_{1}, z_{2}\right)\right)_{i=1}^{n}$ of homogeneous polynomials of degree $d$;

$$
u_{i}\left(z_{1}, z_{2}\right)=\sum_{j=0}^{d} a_{i j} z_{1}^{j} z_{2}^{d-j}, \quad i=1, \ldots, n .
$$

This compacification is called the quasimap space and denoted by $\mathbf{Q}\left(\mathbb{P}^{n-1} ; d\right)$. An element of the quasimap space is called a quasimap.

3.2. A point $\left[z_{1}: z_{2}\right] \in \mathbb{P}^{1}$ is a base point (or singularity) of a quasimap $u$ if $u\left(z_{1}, z_{2}\right)=0$. A quasimap is a genuine map outside of the base locus. If the degree of the base locus is $d^{\prime}$, then a quasimap can be considered as a genuine map of degree $d-d^{\prime}$. However, it is more convenient to think of a quasimap as a morphism to the quotient stack $\left[\mathbb{A}^{n} / \mathbb{G}_{\mathrm{m}}\right]$. By definition, a morphism from $\mathbb{P}^{1}$ to $\left[\mathbb{A}^{n} / \mathbb{G}_{\mathrm{m}}\right]$ is a principal $\mathbb{G}_{\mathrm{m}}$-bundle $P$ over $\mathbb{P}^{1}$ and a $\mathbb{G}_{\mathrm{m}}$-equivariant morphism $\widetilde{u}: P \rightarrow \mathbb{A}^{n}$. It is a quasimap if the generic point of $P$ is mapped to the semi-stable locus $\mathbb{A}^{n} \backslash\{0\}$.

3.3. Let $x \in H^{2}\left(\mathbf{Q}\left(\mathbb{P}^{n-1} ; d\right) ; \mathbb{Z}\right)$ be the ample generator of the cohomology ring of $\mathbf{Q}\left(\mathbb{P}^{n-1} ; d\right) \cong \mathbb{P}^{n(d+1)-1}$, so that

$$
H^{*}\left(\mathbf{Q}\left(\mathbb{P}^{n-1} ; d\right) ; \mathbb{Z}\right) \cong \mathbb{Z}[x] /\left(x^{n(d+1)}\right)
$$

Given a polynomial $P(x) \in \mathbb{C}[x]$, we set

$$
\langle P(x)\rangle_{\mathbb{P}^{n-1}}:=\sum_{d=0}^{\infty} q^{d}\langle P(x)\rangle_{\mathbb{P}^{n-1}, d} \in \mathbb{C} \llbracket q \rrbracket,
$$

where

$$
\langle P(x)\rangle_{\mathbb{P}^{n-1}, d}:=\int_{\mathbf{Q}\left(\mathbb{P}^{n-1} ; d\right)} P(x)
$$

is the integration over the quasimap space. It follows from

$$
\left\langle x^{k}\right\rangle_{\mathbb{P}^{n-1}, d}= \begin{cases}1 & k=n(d+1)-1, \\ 0 & \text { otherwise }\end{cases}
$$

that

$$
\left\langle x^{k}\right\rangle_{\mathbb{P}^{n-1}}= \begin{cases}q^{d} & k=n(d+1)-1 \text { for some } d \in \mathbb{Z}^{\geq 0} \\ 0 & \text { otherwise }\end{cases}
$$


3.4. If we set $G:=\mathbb{G}_{\mathrm{m}}$ and $W:=\mathbb{C}^{n}$ with the action $G \times \mathbb{G}_{\mathrm{m}} \ni(\alpha, \beta):\left(w_{1}, \ldots, w_{n}\right) \mapsto$ $\left(\alpha w_{1}, \ldots, \alpha w_{n}\right)$, then we have $Z_{d}^{\mathrm{vec}}(x)=1$ and $Z_{d}^{\mathrm{mat}}(x)=\left(x^{-d-1}\right)^{n}$, so that (2.2.4) gives the same result as (3.3.5) under the identification

$$
q=e^{t^{\prime}}
$$

3.5. The small quantum cohomology of $\mathbb{P}^{n-1}$ is the free $\mathbb{C} \llbracket q \rrbracket$-module

$$
\mathrm{QH}\left(\mathbb{P}^{n-1}\right):=H^{*}\left(\mathbb{P}^{n-1} ; \mathbb{C} \llbracket q \rrbracket\right)
$$

equipped with multiplication given by

$$
x^{i} \circ x^{j}:=\sum_{k=0}^{n} \sum_{d=0}^{\infty} q^{d}\left\langle I_{0,3, d}\right\rangle\left(x^{i}, x^{j}, x^{k}\right) x^{n-k-1} .
$$

Here

$$
\left\langle I_{0,3, d}\right\rangle(a, b, c):=\int_{\left[\overline{\mathcal{M}}_{0,3}\left(\mathbb{P}^{n-1} ; d\right)\right]^{\mathrm{virt}}} \mathrm{ev}_{1}^{*} a \cup \mathrm{ev}_{2}^{*} b \cup \mathrm{ev}_{3}^{*} c
$$

is the 3-point Gromov-Witten invariant. It is an associative commutative deformation of the classical cohomology ring;

$$
\mathrm{QH}\left(\mathbb{P}^{n-1}\right) /(q) \cong H^{*}\left(\mathbb{P}^{n-1} ; \mathbb{C}\right) \text {. }
$$

Since the virtual dimension of the moduli space of stable maps is given by

$$
\text { virt.dim } \overline{\mathcal{M}}_{g, k}(X ; d)=(1-g)(\operatorname{dim} X-3)+\left\langle c_{1}(X), d\right\rangle+k
$$

in general, one has

$$
\text { virt.dim } \overline{\mathcal{M}}_{0,3}\left(\mathbb{P}^{n-1} ; d\right)=n d+n-1 \text {. }
$$

The 3-point Gromov-Witten invariant in (3.5.2) is non-zero only if

$$
\text { virt.dim } \overline{\mathcal{M}}_{0,3}\left(\mathbb{P}^{n-1} ; d\right)=i+j+k .
$$

Since $0 \leq i, j, k \leq n-1$, one has (3.5.7) only if $d=0, i+j+k=n-1$ or $d=1$, $i+j+k=2 n-1$. This shows that $x^{i} \circ x^{j}=x^{i+j}$ for $i+j \leq n-1$. Since there is a unique line passing through two points on $\mathbb{P}^{n-1}$ in general position, and this line intersects a hyperplane at one point, one has $x \circ x^{n-1}=q$. Hence the ring structure of the quantum cohomology of $\mathbb{P}^{n-1}$ is given by

$$
\mathrm{QH}\left(\mathbb{P}^{n-1}\right) \cong(\mathbb{C} \llbracket q \rrbracket)[x] /\left(x^{n}-q\right) .
$$

We write the ring homomorphism $\mathbb{C}[x] \rightarrow \mathrm{QH}\left(\mathbb{P}^{n-1}\right)$ sending $x$ to $x$ as $P(x) \mapsto \stackrel{\circ}{P}(x)$.

Theorem 3.6. For any $P(x) \in \mathbb{C}[x]$, one has

$$
\langle P(x)\rangle_{\mathbb{P}^{n-1}}=\int_{\mathbb{P}^{n-1}} \stackrel{\circ}{P}(x) .
$$

Proof. Since both sides of (3.6.1) are linear in $P(x) \in \mathbb{C}[x]$, it suffices to show

$$
\left\langle x^{k}\right\rangle_{\mathbb{P}^{n-1}}=\int_{\mathbb{P}^{n-1}} x^{\circ k}
$$

for any $k \in \mathbb{N}$, which is obvious from (3.3.5) and (3.5.8).

Theorem 3.6 is equivalent to the Vafa-Intriligator formula [Vaf91, Int91]: 
Corollary 3.7 (Vafa-Intriligator formula for projective spaces). For any $P(x) \in \mathbb{C}[x]$, one has

$$
\int_{\mathbb{P}^{n-1}} \stackrel{\circ}{P}(x)=\frac{1}{n} \sum_{\lambda^{n}=q} \frac{P(\lambda)}{\lambda^{n-1}},
$$

where the sum is over $\lambda \in \mathbb{C} \llbracket q^{1 / n} \rrbracket$ satisfying $\lambda^{n}=q$.

Proof. Since the integration over the projective space can be written by residue as

$$
\int_{\mathbb{P}^{r-1}} x^{k}=\delta_{r-1, k}=\operatorname{Res} \frac{x^{k} d x}{x^{r}}
$$

one has

$$
\begin{aligned}
\int_{\mathbb{P}^{n-1}} \stackrel{\circ}{P}(x) & =\langle P(x)\rangle_{\mathbb{P}^{n-1}} \\
& =\sum_{d=0}^{\infty} q^{d} \int_{\mathbf{Q}\left(\mathbb{P}^{n-1} ; d\right)} P(x) \\
& =\sum_{d=0}^{\infty} q^{d} \operatorname{Res} \frac{P(x) d x}{x^{n(d+1)}} \\
& =\operatorname{Res} \frac{x^{-n} P(x)}{1-q x^{-n}} \\
& =\operatorname{Res} \frac{P(x)}{x^{n}-q} \\
& =\frac{1}{n} \sum_{\lambda^{n}=q} \frac{P(\lambda)}{\lambda^{n-1}}
\end{aligned}
$$

and (3.7.1) is proved.

3.8. The projective space $\mathbb{P}^{n-1}$ has a natural action of $\mathrm{GL}_{n}$, which restricts to the action of the diagonal maximal torus $H$. The equivariant cohomology is defined as the ordinary cohomology $H_{H}^{*}\left(\mathbb{P}^{n-1}\right):=H^{*}\left(\mathbb{P}_{H}^{n-1}\right)$ of the Borel construction $\mathbb{P}_{H}^{n-1}:=\mathbb{P}^{n-1} \times_{H} E H$, where $E H$ is the product of $n$ copies of the total space of the tautological bundle $\mathcal{O}_{\mathbb{P} \infty}(-1)$ over $B \mathbb{G}_{\mathrm{m}}=\mathbb{P}^{\infty}$. It follows that $\mathbb{P}_{H}^{n-1}$ is the projectivization $\mathbb{P}(\mathcal{E})$ of the vector bundle $\mathcal{E}:=\bigoplus_{i=1}^{n} \pi_{i}^{*} \mathcal{O}_{\mathbb{P} \infty}(-1)$ of rank $n$ over $\left(\mathbb{P}^{\infty}\right)^{n}$. A standard result on the cohomology of a projective bundle (see e.g. [GH78, page 606]) shows that $H^{*}\left(\mathbb{P}_{H}^{n-1}\right)$ is generated over $H_{H}^{*}(\mathrm{pt})=H^{*}\left(\left(\mathbb{P}^{\infty}\right)^{n}\right) \cong \mathbb{C}\left[\lambda_{1}, \ldots, \lambda_{n}\right]$ by $x:=-c_{1}\left(\mathcal{O}_{\mathbb{P}(\mathcal{E})}(-1)\right)$ with one relation

$$
(-x)^{n}-c_{1}(\mathcal{E})(-x)^{n-1}+c_{2}(\mathcal{E})(-x)^{n-2}+\cdots+(-1)^{n} c_{n}(\mathcal{E})=0 .
$$

Since $c_{i}(\mathcal{E})=(-1)^{i} \sigma_{i}\left(\lambda_{1}, \ldots, \lambda_{n}\right)$, one obtains

$$
H_{H}^{*}\left(\mathbb{P}^{n-1}\right) \cong \mathbb{C}\left[x, \lambda_{1}, \ldots, \lambda_{n}\right] / \prod_{i=1}^{n}\left(x-\lambda_{i}\right) .
$$

The $H$-fixed locus $\left(\mathbb{P}^{n-1}\right)^{H}$ consists of $n$ points $\left\{p_{i}\right\}_{i=1}$, where $p_{i}$ is the point $\left[z_{1}: \cdots\right.$ : $\left.z_{n}\right] \in \mathbb{P}^{n-1}$ with $z_{i}=1$ and $z_{j}=0$ for $i \neq j$. Since the tautological bundle $\mathcal{O}_{\mathbb{P}(\mathcal{E})}(-1)$ restricts to $\pi_{i}^{*} \mathcal{O}_{\mathbb{P} \infty}(-1)$ on $\left(p_{i}\right)_{T}=\left(\mathbb{P}^{\infty}\right)^{n}$, one has

$$
\iota_{i}^{*} x=\lambda_{i} .
$$


The push-forward

$$
\int_{\mathbb{P}^{n-1}}^{H}: H_{H}^{*}\left(\mathbb{P}^{n-1}\right) \rightarrow H_{H}^{*}(\mathrm{pt}) \cong \mathbb{C}\left[\lambda_{1}, \ldots, \lambda_{n}\right]
$$

along the natural map $\left(\mathbb{P}^{n-1}\right)_{H} \rightarrow(\mathrm{pt})_{H} \cong B H$ is called the equivariant integration. The localization theorem $\mathrm{AB} 84$ shows

$$
\begin{aligned}
\int_{\mathbb{P}^{n-1}}^{H} P(x) & =\sum_{i=1}^{n} \frac{\iota_{i}^{*} P(x)}{\operatorname{Eul}^{H}\left(N_{p_{i} / \mathbb{P}^{n-1}}\right)} \\
& =\sum_{i=1}^{n} \frac{P\left(\lambda_{i}\right)}{\prod_{j \neq i}\left(\lambda_{i}-\lambda_{j}\right)} \\
& =\operatorname{Res} \frac{P(x) d x}{\prod_{i=1}^{n}\left(x-\lambda_{i}\right)}
\end{aligned}
$$

for any $P(x) \in H_{H}^{*}\left(\mathbb{P}^{n-1}\right)$.

3.9. The quasimap space $\mathbf{Q}\left(\mathbb{P}^{n-1} ; d\right)$ has a natural action of $H \times \mathbb{G}_{\mathrm{m}}$ given by

$$
H \times \mathbb{G}_{\mathrm{m}} \ni\left(\alpha_{1}, \ldots, \alpha_{n}, \beta\right):\left(u_{i}\left(z_{1}, z_{2}\right)\right)_{i=1}^{n} \mapsto\left(\alpha_{i} u_{i}\left(z_{1}, \beta z_{2}\right)\right)_{i=1}^{n} .
$$

The equivariant cohomology of $\mathbf{Q}\left(\mathbb{P}^{n-1} ; d\right)$ with respect to this torus action is given by

$$
H_{H \times \mathbb{G}_{\mathrm{m}}}^{*}\left(\mathbf{Q}\left(\mathbb{P}^{n-1} ; d\right) ; \mathbb{C}\right) \cong \mathbb{C}\left[x, \lambda_{1}, \ldots, \lambda_{n}, \mathrm{z}\right] /\left(\prod_{i=1}^{n} \prod_{j=0}^{d}\left(x-\lambda_{i}-j \mathrm{z}\right)\right) .
$$

The $H \times \mathbb{G}_{\mathrm{m}}$-equivariant integration

$$
\langle-\rangle_{\mathbb{P}^{n-1}, d}^{H \times \mathbb{G}_{\mathrm{m}}}: H_{H \times \mathbb{G}_{\mathrm{m}}}^{*}\left(\mathbf{Q}\left(\mathbb{P}^{n-1} ; d\right) ; \mathbb{C}\right) \rightarrow H^{*}\left(B\left(H \times \mathbb{G}_{\mathrm{m}}\right) ; \mathbb{C}\right)
$$

is given by

$$
\begin{aligned}
\langle P(x)\rangle_{\mathbb{P}^{n-1}, d}^{H \times \mathbb{G}_{\mathrm{m}}} & =\operatorname{Res} \frac{P(x) d x}{\prod_{i=1}^{n} \prod_{j=0}^{d}\left(x-\lambda_{i}-j z\right)} \\
& =\sum_{i=1}^{n} \sum_{j=0}^{d} \frac{P\left(\lambda_{i}+j \mathrm{z}\right)}{\prod_{(k, l) \neq(i, j)}\left(\left(\lambda_{i}+j \mathrm{z}\right)-\left(\lambda_{k}+l \mathbf{z}\right)\right)} .
\end{aligned}
$$

The $H \times \mathbb{G}_{\mathrm{m}}$-equivariant correlator is given by

$$
\langle P(x)\rangle_{\mathbb{P}^{n-1}}^{H \times \mathbb{G}_{\mathrm{m}}}:=\sum_{d=0}^{\infty} q^{d}\langle P(x)\rangle_{\mathbb{P}^{n-1}, d}^{H \times \mathbb{G}_{\mathrm{m}}}
$$

The $H$-equivariant correlator $\langle P(x)\rangle_{\mathbb{P}^{n-1}}^{H}$ and the $\mathbb{G}_{\mathrm{m}}$-equivariant correlator $\langle P(x)\rangle_{\mathbb{P}^{n-1}}^{\mathbb{G}_{\mathrm{m}}}$ are obtained by setting $\mathbf{z}=0$ and $\boldsymbol{\lambda}=\left(\lambda_{1}, \ldots, \lambda_{n}\right)=0$ respectively.

3.10. The fixed point of the $\mathbb{G}_{\mathrm{m}}$-action on $\mathbf{Q}\left(\mathbb{P}^{n-1} ; d\right)$ is the disjoint union

$$
\mathbf{Q}\left(\mathbb{P}^{n-1} ; d\right)^{\mathbb{G}_{\mathrm{m}}}=\coprod_{i=0}^{d} \mathbf{Q}\left(\mathbb{P}^{n-1} ; d\right)_{i}^{\mathbb{G}_{\mathrm{m}}}
$$

of $d+1$ connected components

$$
\mathbf{Q}\left(\mathbb{P}^{n-1} ; d\right)_{i}^{\mathbb{G}_{\mathrm{m}}}:=\left\{\left[a_{1} z_{1}^{i} z_{2}^{d-i}, \ldots, a_{n} z_{1}^{i} z_{2}^{d-i}\right] \in \mathbf{Q}\left(\mathbb{P}^{n-1} ; d\right) \mid\left[a_{1}, \ldots, a_{n}\right] \in \mathbb{P}^{n-1}\right\}
$$


Each of these connected components is isomorphic to $\mathbb{P}^{n-1}$, and the base locus is $i 0+(d-$ i) $\infty$. The connected component $\mathbf{Q}\left(\mathbb{P}^{n-1} ; d\right)_{0}^{\mathbb{G}_{\mathrm{m}}}$ will be denoted by $\mathbf{Q} .\left(\mathbb{P}^{n-1} ; d\right)$. There is a natural map ev: Q. $\left(\mathbb{P}^{n-1} ; d\right) \rightarrow \mathbb{P}^{n-1}$ called the evaluation map, and one has

$$
\mathrm{Q}\left(\mathbb{P}^{n-1} ; d\right)^{\mathbb{G}_{\mathrm{m}}} \cong \coprod_{d_{1}+d_{2}=d} \mathrm{Q}_{\bullet}\left(\mathbb{P}^{n-1} ; d_{1}\right) \times_{\mathbb{P}^{n-1}} \mathrm{Q}_{\bullet}\left(\mathbb{P}^{n-1} ; d_{2}\right) .
$$

The normal bundle of Q. $\left(\mathbb{P}^{n-1} ; d\right)$ in $\mathbf{Q}\left(\mathbb{P}^{n-1} ; d\right)$ is given by $\mathcal{O}_{\mathbb{P}^{n-1}}(1)^{\oplus n d}$, whose equivariant Euler class is given by

$$
\operatorname{Eul}^{H \times \mathbb{G}_{\mathrm{m}}}\left(N_{\mathbf{Q} .\left(\mathbb{P}^{n-1} ; d\right) / \mathbf{Q}\left(\mathbb{P}^{n-1} ; d\right)}\right)=\prod_{i=1}^{n} \prod_{l=1}^{d}\left(x-\lambda_{i}+l \mathbf{z}\right) .
$$

The equivariant I-function is defined by

$$
I_{\mathbb{P}^{n-1}}^{H}(t ; \mathbf{z}):=e^{t x / z} \sum_{d=0}^{\infty} e^{d t} I_{d}^{H}
$$

where

$$
\begin{aligned}
I_{d}^{H}(\mathrm{z}) & :=\mathrm{ev}_{*}\left(\frac{1}{\operatorname{Eul}^{H \times \mathbb{G}_{\mathrm{m}}}\left(N_{\mathbf{Q}_{\mathbf{0}}\left(\mathbb{P}^{n-1} ; d\right) / \mathbf{Q}\left(\mathbb{P}^{n-1} ; d\right)}\right)}\right) \\
& =\frac{1}{\prod_{i=1}^{n} \prod_{l=1}^{d}\left(x-\lambda_{i}+l \mathbf{z}\right)} .
\end{aligned}
$$

The non-equivariant $I$-function is defined similarly, and given by setting $\boldsymbol{\lambda}=0$ in (3.10.5);

$$
I_{\mathbb{P}^{n-1}}(t ; \mathbf{z}):=e^{t x / z} \sum_{d=0}^{\infty} \frac{e^{d t}}{\prod_{l=1}^{d}(x+l \mathbf{z})^{n}} .
$$

3.11. Let $\left(\mathbb{C} \llbracket e^{t} \rrbracket\right)[t]$ be the polynomial ring in $t$ with the ring $\mathbb{C} \llbracket e^{t} \rrbracket$ of formal power series in $e^{t}$ as a coefficient. The equivariant $I$-function in (3.10.5) is an element of $H_{H}^{*}\left(\mathbb{P}^{n-1} ; \mathbb{C}\right) \otimes_{\mathbb{C}}\left(\mathbb{C} \llbracket e^{t} \rrbracket\right)[t]$, and the variable $t$ is related to the variable $q$ appearing in the correlator by

$$
q=e^{t}
$$

The equivariant $I$-function can also be considered as a $H_{H}^{*}\left(\mathbb{P}^{n-1} ; \mathbb{C}\right)$-valued analytic function, which is multi-valued as a function of $q$ and single-valued as a function of $t=\log q$.

3.12. There is a $\mathbb{G}_{\mathrm{m}}$-equivariant evaluation map ev $: \mathrm{Q}\left(\mathbb{P}^{n-1} ; d\right) \rightarrow\left[\mathbb{C}^{n} / \mathbb{G}_{\mathrm{m}}\right]$ at the point $0 \in \mathbb{P}^{1}$. By abuse of notation, we also let $x$ denote the $\mathbb{G}_{\mathrm{m}}$-equivariant Euler class of the line bundle $\operatorname{ev}_{0}^{*}\left(\mathcal{O}_{\left[\mathbb{C}^{n} / \mathbb{G}_{\mathrm{m}}\right]}(1)\right)$. Here $\mathcal{O}_{\left[\mathbb{C}^{n} / \mathbb{G}_{\mathrm{m}}\right]}(1)$ is the line bundle $\left[\left(\mathbb{C}^{n} \times \mathbb{C}\right) / \mathbb{G}_{\mathrm{m}}\right]$ on the quotient stack with weights $((1, \ldots, 1), 1)$.

Let $\iota_{i}: \mathbf{Q}\left(\mathbb{P}^{n-1} ; d\right)_{i}^{\mathbb{G}_{\mathrm{m}}} \rightarrow \mathbf{Q}\left(\mathbb{P}^{n-1} ; d\right)$ be the inclusion of the $i$-th connected component (3.10.2). Since $\iota_{i}^{*}(x)=x+i z$ (under the identification $\mathbf{Q}\left(\mathbb{P}^{n-1} ; d\right)_{i}^{\mathbb{G}_{\mathrm{m}}}=\mathbb{P}^{n-1}$ ) and

$$
\frac{1}{\operatorname{Eul}^{\mathbb{G}_{\mathrm{m}}}\left(N_{\mathbf{Q}\left(\mathbb{P}^{n-1} ; d\right)_{i}^{\mathbb{G}_{\mathrm{m}}} / \mathbf{Q}\left(\mathbb{P}^{n-1} ; d\right)}\right)}=I_{i}(\mathbf{z}) \cup I_{d-i}(-\mathbf{z}),
$$


localization with respect to the $\mathbb{G}_{\mathrm{m}}$-action shows that

$$
\begin{aligned}
\sum_{d=0}^{\infty} e^{d \tau}\left\langle e^{(t-\tau) x / \mathbf{z}}\right\rangle_{\mathbb{P}^{n-1}, d}^{\mathbb{G}_{\mathrm{m}}} & =\sum_{d=0}^{\infty} e^{d \tau} \sum_{i=0}^{d} \int_{\mathbf{Q}\left(\mathbb{P}^{n-1} ; d\right)_{i}^{\mathbb{G}_{\mathrm{m}}}} \frac{\iota_{i}^{*}\left(e^{(t-\tau) x / \mathbf{z}}\right)}{\operatorname{Eul}^{\mathbb{G}_{\mathrm{m}}}\left(N_{\mathbf{Q}\left(\mathbb{P}^{n-1} ; d\right)_{i}^{\mathbb{G}_{\mathrm{m}}} / \mathbf{Q}\left(\mathbb{P}^{n-1} ; d\right)}\right)} \\
& =\sum_{d=0}^{\infty} e^{d \tau} \sum_{i=0}^{d} \int_{\mathbb{P}^{n-1}} e^{(t-\tau)(x+i \mathbf{z}) / \mathbf{z}} \cup I_{i}(\mathbf{z}) \cup I_{d-i}(-\mathbf{z}) \\
& =\sum_{d=0}^{\infty} \sum_{i=0}^{d} \int_{\mathbb{P}^{n-1}} e^{t x / \mathbf{z}} e^{t i} I_{i}(\mathbf{z}) \cup e^{-\tau x / \mathbf{z}} e^{(d-i) \tau} I_{d-i}(-\mathbf{z}) \\
& =\int_{\mathbb{P}^{n-1}} I_{\mathbb{P}^{n-1}}(t ; \mathbf{z}) \cup I_{\mathbb{P}^{n-1}}(\tau ;-\mathbf{z}) .
\end{aligned}
$$

The factorization of the $H \times \mathbb{G}_{\mathrm{m}}$-equivariant correlator is proved similarly as

$$
\begin{aligned}
& \sum_{d=0}^{\infty} e^{d \tau}\left\langle e^{(t-\tau) x / \mathrm{z}}\right\rangle_{\mathbb{P}^{n-1}, d}^{H \times \mathbb{G}_{\mathrm{m}}} \\
& =\sum_{d=0}^{\infty} \operatorname{Res} \frac{e^{d \tau} e^{(t-\tau) x / z} d x}{\prod_{i=1}^{n} \prod_{l=0}^{d}\left(x-\lambda_{i}-l \mathbf{z}\right)} \\
& =\sum_{d=0}^{\infty} \sum_{m=0}^{d} \sum_{j=1}^{n} \operatorname{Res}_{x=\lambda_{j}+m z} \frac{e^{d \tau} e^{(t-\tau) x / z} d x}{\prod_{i=1}^{n} \prod_{l=0}^{d}\left(x-\lambda_{i}-l \mathbf{z}\right)} \\
& =\sum_{d=0}^{\infty} \sum_{m=0}^{d} \sum_{j=1}^{n} \operatorname{Res}_{x=\lambda_{j}} \frac{e^{d \tau} e^{(t-\tau) x / \mathbf{z}} e^{(t-\tau) m} d x}{\prod_{i=1}^{n} \prod_{l=0}^{d}\left(x-\lambda_{i}-(l-m) \mathrm{z}\right)} \\
& =\sum_{d=0}^{\infty} \sum_{m=0}^{d} \sum_{j=1}^{n} \operatorname{Res}_{x=\lambda_{j}} \frac{e^{t x / \mathbf{z}} e^{m t}}{\prod_{i=1}^{n} \prod_{l=1}^{m}\left(x-\lambda_{i}+l \mathbf{z}\right)} \frac{e^{-\tau x / \mathbf{z}} e^{(d-m) \tau}}{\prod_{i=1}^{n} \prod_{l=1}^{d-m}\left(x-\lambda_{i}-l \mathbf{z}\right)} \frac{d x}{\prod_{i=1}^{n}\left(x-\lambda_{i}\right)} \\
& =\sum_{d=0}^{\infty} \sum_{d^{\prime}=0}^{\infty} \sum_{j=1}^{n} \operatorname{Res}_{x=\lambda_{j}} \frac{e^{t x / \mathbf{z}} e^{d t}}{\prod_{i=1}^{n} \prod_{l=1}^{d}\left(x-\lambda_{i}+l \mathbf{z}\right)} \frac{e^{-\tau x / \mathbf{z}} e^{d^{\prime} \tau}}{\prod_{i=1}^{n} \prod_{l=1}^{d^{\prime}}\left(x-\lambda_{i}-l \mathbf{z}\right)} \frac{d x}{\prod_{i=1}^{n}\left(x-\lambda_{i}\right)} \\
& =\int_{\mathbb{P}^{n-1}}^{H} I_{\mathbb{P}^{n-1}}^{H}(t ; \mathbf{z}) \cup I_{\mathbb{P}^{n-1}}^{H}(\tau ;-\mathbf{z}) \text {. }
\end{aligned}
$$

This can also be regarded as a purely combinatorial proof.

3.13. Let ev: $\overline{\mathcal{M}}_{0,1}\left(\mathbb{P}^{n-1} ; d\right) \rightarrow \mathbb{P}^{n-1}$ be the evaluation map from the moduli space of stable maps of genus 0 and degree $d$ with 1 marked point, and $\psi$ be the first Chern class of the line bundle over $\overline{\mathcal{M}}_{0,1}\left(\mathbb{P}^{n-1} ; d\right)$ whose fiber at a stable map $\varphi:(C, x) \rightarrow \mathbb{P}^{n-1}$ is the cotangent line $T_{x}^{*} C$ at the marked point. The equivariant $J$-function [Giv96] is a $H^{*}\left(\mathbb{P}^{n-1} ; \mathbb{C}\right)$-valued hypergeometric series given by

$$
J_{\mathbb{P}^{n-1}}^{H}(t ; \mathbf{z}):=e^{t x / \mathbf{z}} \sum_{d=0}^{\infty} e^{d t} J_{d}
$$

where

$$
J_{d}:=\operatorname{ev}_{*}\left(\frac{1}{z(z-\psi)}\right) .
$$


3.14. The graph space is defined by $G\left(\mathbb{P}^{n-1} ; d\right):=\overline{\mathcal{M}}_{0,0}\left(\mathbb{P}^{n-1} \times \mathbb{P}^{1} ;(d, 1)\right)$. The source of any map $\varphi: C \rightarrow \mathbb{P}^{n-1} \times \mathbb{P}^{1}$ in $G\left(\mathbb{P}^{n-1} ; d\right)$ has a distinguished irreducible component $C_{1}$ which maps isomorphically to $\mathbb{P}^{1}$. Let $G\left(\mathbb{P}^{n-1} ; d\right)_{0}$ be the open subspace of $G\left(\mathbb{P}^{n-1} ; d\right)$ consisting of stable maps without irreducible components mapping constantly to $0 \in \mathbb{P}^{1}$. There is a map ev ${ }_{0}: G\left(\mathbb{P}^{n-1} ; d\right)_{0} \rightarrow \mathbb{P}^{n-1}$ sending $\varphi: C \rightarrow \mathbb{P}^{n-1} \times \mathbb{P}^{1}$ to $\operatorname{pr}_{1} \circ \varphi\left(\left(\operatorname{pr}_{2} \circ \varphi\right)^{-1}(0)\right)$. The fixed locus of the natural $\mathbb{G}_{\mathrm{m}}$-action on $G\left(\mathbb{P}^{n-1} ; d\right)_{0}$ can be identified with $\overline{\mathcal{M}}_{0,1}\left(\mathbb{P}^{n-1} ; d\right)$. Since the natural morphism $G\left(\mathbb{P}^{n-1} ; d\right)_{0} \rightarrow \mathbf{Q}\left(\mathbb{P}^{n-1} ; d\right)_{0}$ is a $\mathbb{G}_{\mathrm{m}}$-equivariant birational morphism which commutes with the evaluation maps, the push-forwards $J_{d}$ and $I_{d}$ of 1 by ev ev $_{0}: G\left(\mathbb{P}^{n-1} ; d\right)_{0} \rightarrow \mathbb{P}^{n-1}$ and ev: $\mathbf{Q}\left(\mathbb{P}^{n-1} ; d\right)_{0} \rightarrow \mathbb{P}^{n-1}$ are equal, and hence $I_{\mathbb{P}^{n-1}}(t ; \mathbf{z})=J_{\mathbb{P}^{n-1}}(t ; \mathbf{z})$.

3.15. The effective potential (2.4.1) is given by

$$
W_{\mathrm{eff}}(x ; t)=t x-\sum_{i=1}^{n}\left(x-\lambda_{i}\right)\left(\log \left(x-\lambda_{i}\right)-1\right) .
$$

One can easily see

$$
\begin{aligned}
& \frac{\partial W_{\text {eff }}}{\partial x}=t-\sum_{i=1}^{n} \log \left(x-\lambda_{i}\right), \\
& e^{\partial_{x} W_{\text {eff }}}=q \prod_{i=1}^{n}\left(x-\lambda_{i}\right)^{-1},
\end{aligned}
$$

so that

$$
\langle P(x)\rangle_{\mathbb{P}^{n-1}}^{H}=\operatorname{Res} \frac{P(x) d x}{\prod_{i=1}^{n}\left(x-\lambda_{i}\right)\left(1-e^{\left.\partial_{x} W_{\text {eff }}\right)}\right.} .
$$

Note that the equation

$$
e^{\partial_{x} W_{\text {eff }}}=1
$$

gives the relation

$$
\prod_{i=1}^{n}\left(x-\lambda_{i}\right)=q
$$

in the equivariant quantum cohomology of $\mathbb{P}^{n-1}$.

\section{Projective Complete intersections}

4.1. Let $f_{1}\left(w_{1}, \ldots, w_{n}\right), \ldots, f_{r}\left(w_{1}, \ldots, w_{n}\right) \in \mathbb{C}\left[w_{1}, \ldots, w_{n}\right]$ be homogeneous polynomials of degrees $l_{1}, \ldots, l_{r}$ satisfying the Calabi-Yau condition

$$
l_{1}+\cdots+l_{r}=n .
$$

Assume that $f_{1}, \ldots, f_{r}$ are sufficiently general so that

$$
Y:=\left\{\left[w_{1}, \cdots, w_{n}\right] \in \mathbb{P}^{n-1} \mid f_{1}\left(w_{1}, \ldots, w_{n}\right)=\cdots=f_{r}\left(w_{1}, \ldots, w_{n}\right)=0\right\}
$$

is a smooth complete intersection of dimension $n-r-1$, whose Poincaré dual is

$$
v:=\prod_{i=1}^{r}\left(l_{i} x\right) .
$$


Define the quasimap space $\mathbf{Q}(Y ; d)$ as the subset of $\mathbf{Q}\left(\mathbb{P}^{n-1} ; d\right)$ consisting of $\left[\varphi_{1}\left(z_{1}, z_{2}\right), \ldots, \varphi_{n}\left(z_{1}, z_{2}\right)\right]$ satisfying

(4.1.4) $f_{i}\left(\varphi_{1}\left(z_{1}, z_{2}\right), \ldots, \varphi_{n}\left(z_{1}, z_{2}\right)\right)=0 \in \mathbb{C}\left[z_{1}, z_{2}\right]$ for any $i \in\{1, \ldots, r\}$.

Since $f_{i}\left(\varphi_{1}\left(z_{1}, z_{2}\right), \ldots, \varphi_{n}\left(z_{1}, z_{2}\right)\right) \in \mathbb{C}\left[z_{1}, z_{2}\right]$ is a homogeneous polynomial of degree $d l_{i}$ in $z_{1}$ and $z_{2}$, it contains $d l_{i}+1$ terms, each of which is a homogeneous polynomial of degree $l_{i}$ in $\left(a_{k l}\right)_{k, l}$. With this in mind, the Morrison-Plesser class is defined by

$$
\Phi(Y ; d):=\prod_{i=1}^{r}\left(l_{i} x\right)^{l_{i} d} \in H^{*}\left(\mathbf{Q}\left(\mathbb{P}^{n-1} ; d\right) ; \mathbb{Z}\right),
$$

so that $\Phi(Y ; d) \cup v$ is the Poincaré dual of $[\mathbf{Q}(Y ; d)]^{\text {virt }} \in H_{*}\left(\mathbf{Q}\left(\mathbb{P}^{n-1} ; d\right) ; \mathbb{Z}\right)$. If we set

$$
\langle P(x)\rangle_{Y, d}:=\int_{\mathbf{Q}\left(\mathbb{P}^{n-1} ; d\right)} P(x) \cup \Phi(Y ; d) \cup v
$$

and

$$
\langle P(x)\rangle_{Y}:=\sum_{d=0}^{\infty} q^{d}\langle P(x)\rangle_{Y, d}
$$

for $P(x) \in \mathbb{C}[x]$, then we have

$$
\begin{aligned}
\left\langle x^{n-r-1}\right\rangle_{Y} & =\sum_{d=0}^{\infty} q^{d} \operatorname{Res} \frac{x^{n-r-1} \Phi(Y, d) v d x}{x^{n(d+1)}} \\
& =\sum_{d=0}^{\infty} q^{d} \operatorname{Res} \frac{x^{n-r-1} \prod_{i=1}^{r}\left(l_{i} x\right)^{l_{i} d+1} d x}{x^{n(d+1)}} \\
& =\sum_{d=0}^{\infty} q^{d} \prod_{i=1}^{r}\left(l_{i}\right)^{l_{i} d+1} \\
& =\frac{\prod_{i=1}^{r} l_{i}}{1-q \prod_{i=1}^{r}\left(l_{i}\right)^{l_{i}}} .
\end{aligned}
$$

4.2. The gauged linear sigma model for $Y$ is obtained from the gauged linear sigma model for $\mathbb{P}^{n-1}$ by adding $r$ fields of $G=\mathbb{G}_{\mathrm{m}}$-charge $-l_{1}, \ldots,-l_{r}$ and R-charge 2 . One has $Z_{d}^{\mathrm{vec}}(x)=1$ and $Z_{d}^{\mathrm{mat}}(x)=\left(x^{-d-1}\right)^{n} \cdot \prod_{i=1}^{r}\left(-l_{i} x\right)^{l_{i} d+1}$ in this case, so that (2.2.4) gives

$$
\begin{aligned}
\sum_{d=0}^{\infty} e^{t^{\prime} d} \operatorname{Res}\left(x^{-d-1}\right)^{n} \prod_{i=1}^{r}\left(-l_{i} x\right)^{l_{i} d+1} x^{n-r-1} & =\sum_{d=0}^{\infty} e^{t^{\prime} d} \prod_{i=1}^{r}\left(-l_{i}\right)^{l_{i} d+1} \\
& =\sum_{d=0}^{\infty}(-1)^{\sum_{i=1}^{r} l_{i} d} e^{t^{\prime} d} \prod_{i=1}^{r}\left(l_{i}\right)^{l_{i} d+1} \\
& =\sum_{d=0}^{\infty}\left((-1)^{n} e^{t^{\prime}}\right)^{d} \prod_{i=1}^{r}\left(l_{i}\right)^{l_{i} d+1}
\end{aligned}
$$

which coincides with (4.1.8) under the identification

$$
q=(-1)^{n} e^{t^{\prime}}
$$


4.3. The mirror $\check{Y}$ of $Y$ is a compactification of a complete intersection in $\mathbb{C}^{n}$ defined by

$$
\begin{aligned}
\check{f}_{1} & :=1-\left(a_{1} \check{y}_{1}+\cdots+a_{l_{1}} \check{y}_{l_{1}}\right), \\
\check{f}_{2} & :=1-\left(a_{l_{1}+1} \check{y}_{l_{1}+1}+\cdots+a_{l_{1}+l_{2}} \check{y}_{l_{1}+l_{2}}\right), \\
& \vdots \\
\check{f}_{r} & :=1-\left(a_{l_{1}+\cdots+l_{i-1}+1} \check{y}_{l_{1}+\cdots+l_{i-1}+1}+\cdots+a_{n} \check{y}_{n}\right), \\
\check{f}_{0} & :=\check{y}_{1} \cdots \check{y}_{n}-1 .
\end{aligned}
$$

The complex structure of $\check{Y}$ depends not on the individual $a_{i}$ but only on $\alpha=a_{1} \cdots a_{n}$. The Yukawa (n-2)-point function is defined by

$$
\mathcal{Y}(\alpha):=\frac{(-1)^{(n-1)(n-2) / 2}}{(2 \pi \sqrt{-1})^{n-1}} \int_{\check{Y}} \Omega \wedge\left(\alpha \frac{\partial}{\partial \alpha}\right)^{n-2} \Omega,
$$

where

$$
\Omega:=\operatorname{Res}\left(\frac{d \check{y}_{1} \wedge \cdots \wedge d \check{y}_{n}}{\check{f}_{0} \check{f}_{1} \cdots \check{f}_{r}}\right)
$$

is the holomorphic volume form on $\check{Y}$. The computation in BvS95, Proposition 5.1.2] shows

$$
\mathcal{Y}(\alpha)=\frac{\prod_{i=1}^{r} l_{i}}{1-\alpha \prod_{i=1}^{r}\left(l_{i}\right)^{l_{i}}},
$$

which coincides with (4.1.11) under the identification $q=\alpha$ of variables;

$$
\mathcal{Y}(\alpha)=\left.\left\langle x^{n-r-1}\right\rangle_{Y}\right|_{q=\alpha} .
$$

A generalization of (4.3.9) to toric complete intersections is toric residue mirror symmetry conjectured in [BM02, BM03] and proved in [SV04, Bor05, Kar05, SV06].

\section{Concave Bundles on Projective spaCes}

5.1. Let $l_{1}, l_{2}, \cdots, l_{r}$ be positive integers and

$$
Y:=\operatorname{Spec}_{\mathbb{P}^{n-1}}\left(\mathcal{S}_{m^{*}}{ }^{\vee} \mathcal{E}^{\vee}\right)
$$

be the total space of the vector bundle associated with the locally free sheaf

$$
\mathcal{E}:=\mathcal{O}_{\mathbb{P}^{n-1}}\left(-l_{1}\right) \oplus \cdots \oplus \mathcal{O}_{\mathbb{P}^{n-1}}\left(-l_{r}\right)
$$

on $\mathbb{P}^{n-1}$. Since any holomorphic map from $\mathbb{P}^{1}$ to $Y$ of positive degree $d$ factors through the zero-section $\mathbb{P}^{n-1} \rightarrow Y$, we define the quasimap space to $Y$ as

$$
\mathbf{Q}(Y ; d):=\mathbf{Q}\left(\mathbb{P}^{n-1} ; d\right) .
$$

5.2. To equip $\mathbf{Q}(Y ; d)$ with a natural obstruction theory, we identify $\mathbf{Q}(Y ; d)$ with an open substack of the mapping stack $\operatorname{Map}\left(\mathbb{P}^{1}, \mathcal{Y}\right)$ to the quotient stack

$$
\mathcal{Y}:=\left[\left(\mathbb{A}^{n} \times \mathbb{A}^{r}\right) / \mathbb{G}_{\mathrm{m}}\right]
$$

of $\mathbb{A}^{n} \times \mathbb{A}^{r}$ by the $\mathbb{G}_{\mathrm{m}}$-action given by

$$
\mathbb{G}_{\mathrm{m}} \ni \alpha:\left(x_{1}, \ldots, x_{n}, z_{1}, \ldots, z_{r}\right) \mapsto\left(\alpha x_{1}, \ldots, \alpha x_{n}, \alpha^{-l_{1}} z_{1}, \ldots, \alpha^{-l_{r}} z_{r}\right) .
$$


A morphism $\mathbb{P}^{1} \rightarrow \mathcal{Y}$ consists of a line bundle $\mathcal{L}$ on $\mathbb{P}^{1}$ and sections

$$
\left(\left(\varphi_{i}\right)_{i=1}^{n},\left(\psi_{j}\right)_{j=1}^{r}\right) \in\left(\left(H^{0}(\mathcal{L})\right)^{n} \times \prod_{j=1}^{r} H^{0}\left(\mathcal{L}^{\otimes\left(-l_{j}\right)}\right)\right),
$$

whose degree is defined as the degree of $\mathcal{L}$.

5.3. Recall from [BF97, Definition 4.4] that an obstruction theory for a Deligne-Mumford stack $\mathcal{X}$ is a morphism $\phi: E \rightarrow L_{\mathcal{X}}$ from an object $E$ of the derived category of quasicoherent sheaves on $\mathcal{X}$ satisfying

(1) $h^{i}(E) \cong 0$ for $i>0$, and

(2) $h^{i}(E)$ is coherent for $i=0,-1$

to the cotangent complex $L_{\mathcal{X}}$ such that

(1) $h^{0}(\phi)$ is an isomorphism, and

(2) $h^{-1}(\phi)$ is an epimorphism.

It is said to be perfect if $E$ is locally isomorphic to a two-term complex of locally free sheaves of finite rank [BF97, Definition 5.1].

5.4. A perfect obstruction theory produces the virtual fundamental cycle $[\mathcal{X}]^{\text {virt }}$ in the Chow group $A_{\text {virt.dim }}(\mathcal{X})$ of degree

$$
\text { virt.dim } \mathcal{X}=\operatorname{rank} h^{0}(E)-\operatorname{rank} h^{-1}(E) .
$$

When $\mathcal{X}$ is a smooth scheme, then the cotangent complex $L_{\mathcal{X}}$ is isomorphic to the sheaf $\Omega_{\mathcal{X}}$ of Kähler differentials, and the virtual fundamental cycle is the Euler class of $h^{-1}(E)$.

5.5. The derived mapping stack $\mathbb{R M a p}(\mathcal{S}, \mathcal{T})$ from a proper scheme $\mathcal{S}$ to a derived Artin stack $\mathcal{T}$ is a derived Artin stack (see e.g. [Toë14, Corollary 3.3]) whose tangent complex is given by

$$
T_{\mathbb{R M a p}(\mathcal{S}, \mathcal{T})} \cong \mathbb{R} \pi_{*}\left(\mathbb{L e v}{ }^{*} T_{\mathcal{T}}\right),
$$

where

$$
\pi: \operatorname{RMap}(\mathcal{S}, \mathcal{T}) \times \mathcal{S} \rightarrow \mathbb{R M a p}(\mathcal{S}, \mathcal{T})
$$

is the first projection and

$$
\text { ev }: \mathbb{R M a p}(\mathcal{S}, \mathcal{T}) \times \mathcal{S} \rightarrow \mathcal{T}
$$

is the evaluation morphism. It is a derived thickening of the mapping stack $\operatorname{Map}(\mathcal{S}, \mathcal{T})$, and the pull-back

$$
j^{*}: j^{*} L_{\mathbb{R M a p}(\mathcal{S}, \mathcal{T})} \rightarrow L_{\mathrm{Map}(\mathcal{S}, \mathcal{T})}
$$

by the structure morphism

$$
j: \operatorname{Map}(\mathcal{S}, \mathcal{T}) \rightarrow \mathbb{R} \operatorname{Map}(\mathcal{S}, \mathcal{T})
$$

gives an obstruction theory on $\operatorname{Map}(\mathcal{S}, \mathcal{T})$.

5.6. The restriction of the natural obstruction theory for $\operatorname{Map}\left(\mathbb{P}^{1}, \mathcal{Y}\right)$ to the open substack $\mathbf{Q}(Y ; d)$ gives an obstruction theory for $\mathbf{Q}(Y ; d)$ with $E=\left.j^{*} L_{\mathbb{R M a p}\left(\mathbb{P}^{1}, \mathcal{Y}\right)}\right|_{\mathbf{Q}(Y ; d)}$ and $\phi=\left.j^{*}\right|_{\mathbf{Q}(Y ; d)}$. 
5.7. Since $\operatorname{Pic}\left(\mathbb{A}^{n} \times \mathbb{A}^{r}\right)$ is trivial, the Picard group $\operatorname{Pic} \mathcal{Y} \cong \operatorname{Pic}{ }^{\mathbb{G}_{\mathrm{m}}}\left(\mathbb{A}^{n} \times \mathbb{A}^{r}\right)$ can be identified with the group of characters of $\mathbb{G}_{\mathrm{m}}$, which is non-canonically isomorphic to $\mathbb{Z}$. We fix an isomorphism in such a way that $\bigoplus_{a=0}^{\infty} H^{0}\left(\mathcal{O}_{\mathcal{Y}}(a)\right)$ is the coordinate ring of $\mathbb{A}^{n}$, where $\mathcal{O}_{\mathcal{Y}}(a)$ is the line bundle associated with $a \in \mathbb{Z} \cong \operatorname{Pic} \mathcal{Y}$. Since $\mathcal{Y}$ is the quotient stack of $\mathbb{A}^{n} \times \mathbb{A}^{r}$ by the action of $\mathbb{G}_{\mathrm{m}}$, the tangent complex $T_{\mathcal{Y}}$ satisfies

$$
\varpi^{*} T_{\mathcal{Y}} \cong \text { Cone }\left(\mathcal{O}_{\mathbb{A}^{n} \times \mathbb{A}^{r}} \otimes \operatorname{Lie}\left(\mathbb{G}_{\mathrm{m}}\right) \rightarrow T_{\mathbb{A}^{n} \times \mathbb{A}^{r}}\right)
$$

where $\varpi: \mathbb{A}^{n} \times \mathbb{A}^{r} \rightarrow \mathcal{Y}$ is the quotient morphism. This in turn implies that

$$
T_{\mathcal{Y}} \cong \text { Cone }\left(\mathcal{O}_{\mathcal{Y}} \rightarrow \mathcal{O}_{\mathcal{Y}}(1)^{\oplus n} \oplus \bigoplus_{i=1}^{r} \mathcal{O}_{\mathcal{Y}}\left(-l_{i}\right)\right)
$$

5.8. We write the restriction of the evaluation morphism $\operatorname{Map}\left(\mathbb{P}^{1}, \mathcal{Y}\right) \times \mathbb{P}^{1} \rightarrow \mathcal{Y}$ to the open substack $\mathbf{Q}(Y ; d) \subset \operatorname{Map}\left(\mathbb{P}^{1}, \mathcal{Y}\right)$ as

$$
\text { ev : } \mathbf{Q}(Y ; d) \times \mathbb{P}^{1} \rightarrow \mathcal{Y}
$$

again by abuse of notation. We have

$$
\mathrm{ev}^{*} \mathcal{O}_{\mathcal{Y}}(1) \cong \mathcal{O}_{\mathbf{Q}(Y ; d)}(1) \otimes \mathcal{O}_{\mathbb{P}^{1}}(d)
$$

essentially by definition, where $\mathcal{O}_{\mathbf{Q}(Y ; d)}(1)$ is the ample generator of the Picard group of $\mathbf{Q}(Y ; d) \cong \mathbb{P}^{n(d+1)-1}$. The dual of the natural obstruction theory is given by

$$
\phi^{\vee}: T_{\mathbf{Q}(Y ; d)} \rightarrow E^{\vee}:=\mathbb{R} \pi_{*} \mathrm{ev}^{*} T_{\mathcal{Y}} .
$$

Note that (the inverse of) the isomorphism $h^{0}\left(\phi^{\vee}\right)$ from $T_{\mathbf{Q}(Y ; d)}$ to

$$
\begin{aligned}
h^{0}\left(E^{\vee}\right) & \cong R^{0} \pi_{*} \text { Cone }\left(\mathcal{O}_{\mathbf{Q}(Y ; d) \times \mathbb{P}^{1}} \rightarrow\left(\mathcal{O}_{\mathbf{Q}(Y ; d)}(1) \otimes \mathcal{O}_{\mathbb{P}^{1}}(d)\right)^{\oplus n}\right) \\
& \cong \text { Cone }\left(\mathcal{O}_{\mathbf{Q}(Y ; d)} \rightarrow \mathcal{O}_{\mathbf{Q}(Y ; d)}(1)^{\oplus n(d+1)}\right)
\end{aligned}
$$

gives the Euler sequence

$$
0 \rightarrow \mathcal{O}_{\mathbf{Q}(Y ; d)} \rightarrow \mathcal{O}_{\mathbf{Q}(Y ; d)}(1)^{\oplus n(d+1)} \rightarrow T_{\mathcal{Q}(Y ; d)} \rightarrow 0
$$

on $\mathbf{Q}(Y ; d) \cong \mathbb{P}^{n(d+1)-1}$. One has

$$
\begin{aligned}
h^{1}\left(E^{\vee}\right) & \cong R^{1} \pi_{*}\left(\bigoplus_{i=1}^{r} \mathcal{O}_{\mathbf{Q}(Y ; d)}\left(-l_{i}\right) \otimes \mathcal{O}_{\mathbb{P}^{1}}\left(-l_{i} d\right)\right) \\
& \cong \bigoplus_{i=1}^{r} \mathcal{O}_{\mathbf{Q}(Y ; d)}\left(-l_{i}\right) \otimes H^{1}\left(\mathcal{O}_{\mathbb{P}^{1}}\left(-l_{i} d\right)\right) \\
& \cong \bigoplus_{i=1}^{r} \mathcal{O}_{\mathbf{Q}(Y ; d)}\left(-l_{i}\right)^{\oplus\left(l_{i} d-1\right)}
\end{aligned}
$$

and $h^{i}\left(E^{\vee}\right) \cong 0$ for $i \neq 0,1$, so that this obstruction theory is perfect. By [BF97, Proposition 5.6], the resulting virtual fundamental class is given by

$$
[\mathbf{Q}(Y ; d)]^{\mathrm{virt}}=[\mathbf{Q}(Y ; d)] \cap \operatorname{Eul}\left(h^{1}\left(E^{\vee}\right)\right)=[\mathbf{Q}(Y ; d)] \cap \prod_{i=1}^{r}\left(-l_{i} x\right)^{l_{i} d-1} .
$$

5.9. When the degree is zero, the quasimap space $\mathbf{Q}(Y ; 0)$ is naturally isomorphic to $Y$ equipped with the trivial perfect obstruction theory, so that

$$
[\mathbf{Q}(Y ; 0)]_{15}^{\mathrm{virt}}=[Y] .
$$


5.10. For any $P(x) \in \mathbb{C}[x]$, we define

$$
\langle P(x)\rangle_{Y, d}:=\int_{[\mathbf{Q}(Y ; d)]^{\mathrm{virt}}} P(x)
$$

and

$$
\langle P(x)\rangle_{Y}:=\sum_{d=0}^{\infty} q^{d}\langle P(x)\rangle_{Y, d}
$$

It follows that

$$
\begin{aligned}
\langle P(x)\rangle_{Y} & =\sum_{d=0}^{\infty} q^{k} \int_{\mathbb{P}^{n(d+1)-1}} P(x) \prod_{i=1}^{r}\left(-l_{i} x\right)^{l_{i} d-1} \\
& =\sum_{d=0}^{\infty} q^{k} \operatorname{Res} \frac{P(x) \prod_{i=1}^{r}\left(-l_{i} x\right)^{l_{i} d}}{x^{n(d+1)} \prod_{i=1}^{r}\left(-l_{i} x\right)} .
\end{aligned}
$$

5.11. The gauged linear sigma model for $Y$ is obtained from the gauged linear sigma model for $\mathbb{P}^{n-1}$ by adding $r$ fields of $G=\mathbb{G}_{\mathrm{m}}$-charge $-l_{1}, \ldots,-l_{r}$ and R-charge 0 . One has $Z_{d}^{\text {vec }}(x)=1$ and $Z_{d}^{\text {mat }}(x)=\left(x^{-d-1}\right)^{n} \cdot \prod_{i=1}^{r}\left(-l_{i} x\right)^{l_{i} d-1}$ in this case, so that (2.2.4) gives

$$
\langle P(x)\rangle_{\mathrm{GLSM}}=\sum_{d=0}^{\infty} e^{t^{\prime} d} \operatorname{Res}\left(x^{-d-1}\right)^{n} \prod_{i=1}^{r}\left(-l_{i} x\right)^{l_{i} d-1} P(x),
$$

which coincides with (5.10.4) under the identification

$$
q=e^{t^{\prime}}
$$

5.12. If $\left(l_{1}, \ldots, l_{r}\right)$ satisfies the Calabi-Yau condition

$$
l_{1}+\cdots+l_{r}=n,
$$

then (5.10.4) gives

$$
\left\langle x^{k}\right\rangle_{Y}= \begin{cases}\frac{1}{\left(\prod_{i=1}^{r}\left(-l_{i}\right)\right)\left(1-q \prod_{i=1}^{r}\left(-l_{i}\right)^{l_{i}}\right)} & k=n+r \\ 0 & \text { otherwise }\end{cases}
$$

which matches the Yukawa coupling of the mirror (see e.g. [KM10, Example 6.15]).

\section{Classical mirror symmetry FOR tORIC hypersurfaces}

6.1. Let $\boldsymbol{N}:=\mathbb{Z}^{n}$ be a free abelian group of $\operatorname{rank} n$ and $\boldsymbol{M}:=\check{\boldsymbol{N}}:=\operatorname{Hom}(\boldsymbol{N}, \mathbb{Z})$ be the dual group. Let further $(\Delta, \check{\Delta})$ be a polar dual pair of reflexive polytopes in $\boldsymbol{M}$ and $\boldsymbol{N}$.

6.2. Recall that the fan polytope of a fan is defined as the convex hull of primitive generators of one-dimensional cones. Let $(\Sigma, \check{\Sigma})$ be a pair of smooth projective fans whose fan polytopes are $\breve{\Delta}$ and $\Delta$. The associated toric varieties will be denoted by $X:=X_{\Sigma}$ and $\check{X}:=X_{\check{\Sigma}}$. 
6.3. The set of primitive generators of one-dimensional cones of the fan $\Sigma$ will be denoted by

$$
B:=\left\{\boldsymbol{b}_{1}, \ldots, \boldsymbol{b}_{m}\right\} \subset \boldsymbol{N} .
$$

Assume that $B$ generates $\boldsymbol{N}$. One has the fan sequence

$$
0 \rightarrow \boldsymbol{L} \rightarrow \mathbb{Z}^{m} \stackrel{b}{\rightarrow} \boldsymbol{N} \rightarrow 0
$$

and the divisor sequence

$$
0 \rightarrow M \stackrel{\boldsymbol{b}^{\vee}}{\rightarrow} \mathbb{Z}^{m} \rightarrow \check{\boldsymbol{L}} \rightarrow 0
$$

where $\boldsymbol{b}$ sends the $i$ th coordinate vector $e_{i} \in \mathbb{Z}^{m}$ to $\boldsymbol{b}_{i}$. Recall that

$$
\check{\boldsymbol{L}} \cong \operatorname{Pic}(X) \cong H^{2}(X ; \mathbb{Z}), \quad \operatorname{Eff}(X) \subset \boldsymbol{L} \subset \mathbb{Z}^{m},
$$

where $\operatorname{Eff}(X)$ denotes the semigroup of the effective curves (see [BM02, §3]). We write the group ring of $\boldsymbol{M}$ as $\mathbb{C}[\boldsymbol{M}]$ and define $\mathbb{T}:=\boldsymbol{N}_{\mathbb{G}_{\mathrm{m}}}:=$ Spec $\mathbb{C}[\boldsymbol{M}]$. We also set $\check{\mathbb{T}}:=$ $\operatorname{Spec} \mathbb{C}[\boldsymbol{N}], \check{\mathbb{L}}:=\operatorname{Spec} \mathbb{C}[\boldsymbol{L}]$, and $\mathbb{L}:=\operatorname{Spec} \mathbb{C}[\check{\boldsymbol{L}}]$. The fan sequence induces the exact sequences

$$
1 \rightarrow \mathbb{L} \stackrel{\chi}{\rightarrow}\left(\mathbb{G}_{\mathrm{m}}\right)^{m} \rightarrow \mathbb{T} \rightarrow 1
$$

and

$$
1 \rightarrow \check{\mathbb{T}} \rightarrow\left(\mathbb{G}_{\mathrm{m}}\right)^{m} \rightarrow \check{\mathbb{L}} \rightarrow 1
$$

of algebraic tori. We write the $i$-th components of the map $\chi: \mathbb{L} \rightarrow\left(\mathbb{G}_{\mathrm{m}}\right)^{m}$ in $(6.3 .5)$ as $\chi_{i}$, and the affine line $\mathbb{A}^{1}$ equipped with the action of $\mathbb{L}$ through $\chi_{i}$ as $\mathbb{A}_{i}$. Then one has

$$
X \cong\left(\prod_{i=1}^{m} \mathbb{A}_{i}\right) / / \theta \mathbb{L}
$$

for a suitable choice of a character $\theta \in \check{\boldsymbol{L}} \cong \operatorname{Hom}\left(\mathbb{L}, \mathbb{G}_{\mathrm{m}}\right)$. The right-hand side of (6.3.7) denotes the GIT quotient with respect to the linearization determined by $\theta$.

6.4. We define a graded ring $S_{\Delta}:=\bigoplus_{k=0}^{\infty} S_{\Delta}^{k}$ by

$$
S_{\Delta}^{k}:=\bigoplus_{\boldsymbol{m} \in M \cap(k \Delta)} \mathbb{C} \cdot y_{0}^{k} \boldsymbol{y}^{\boldsymbol{m}}
$$

which is a subalgebra of the semigroup ring

$$
\mathbb{C}[\mathbb{N} \times \boldsymbol{M}]=\mathbb{C}\left[y_{0}, \boldsymbol{y}^{ \pm 1}\right]:=\mathbb{C}\left[y_{0}, y_{1}^{ \pm 1}, \ldots, y_{n}^{ \pm 1}\right]
$$

of $\mathbb{N} \times M$. It is the anti-canonical ring of $X$, so that one has $X \cong \operatorname{Proj} S_{\Delta}$ if and only if $X$ is Fano. The ring $S_{\Delta}$ is Cohen-Macaulay with the dualizing module $I_{\Delta}:=\bigoplus_{k=0}^{\infty} I_{\Delta}^{k}$ given by

$$
I_{\Delta}^{k}:=\bigoplus_{\boldsymbol{m} \in M \cap \operatorname{Int}(k \Delta)} \mathbb{C} \cdot y_{0}^{k} \boldsymbol{y}^{\boldsymbol{m}}
$$

where $\operatorname{Int}(k \Delta)$ is the interior of $k \Delta$. 
6.5. For $\boldsymbol{\alpha}=\left(\alpha_{1}, \ldots, \alpha_{m}\right) \in\left(\mathbb{G}_{\mathrm{m}}\right)^{m}$ (this $\left(\mathbb{G}_{\mathrm{m}}\right)^{m}$ can be naturally considered as the dual torus of the big torus of $X_{\Sigma}$ ), we define an element of the group ring $\mathbb{C}[\boldsymbol{N}]$ by

$$
\check{W}_{\boldsymbol{\alpha}}(\check{\boldsymbol{y}}):=\sum_{i=1}^{m} \alpha_{i} \check{\boldsymbol{y}}^{\boldsymbol{b}_{i}} \in \mathbb{C}[\boldsymbol{N}] .
$$

An element $\check{f} \in \mathbb{C}[\boldsymbol{N}]$ is said to be $\check{\Delta}$-regular if

$$
\check{F}:=\left(\check{F}_{0}, \check{F}_{1}, \ldots, \check{F}_{n}\right):=\left(\check{y}_{0} \check{f}, \check{y}_{0} \check{y}_{1} \partial_{\check{y}_{1}} \check{f}, \ldots, \check{y}_{0} \check{y}_{n} \partial_{\check{y}_{n}} \check{f}\right)
$$

is a regular sequence in $S_{\check{\Delta}}$. We write

$$
\left(\left(\mathbb{G}_{\mathrm{m}}\right)^{m}\right)^{\mathrm{reg}}:=\left\{\boldsymbol{\alpha} \in\left(\mathbb{G}_{\mathrm{m}}\right)^{m} \mid \check{f}_{\boldsymbol{\alpha}}:=1-\check{W}_{\boldsymbol{\alpha}}(\check{\boldsymbol{y}}) \text { is } \check{\Delta} \text {-regular }\right\} .
$$

6.6. Let $\widetilde{\varphi}: \widetilde{\mathfrak{Y}} \rightarrow\left(\left(\mathbb{G}_{\mathrm{m}}\right)^{m}\right)^{\text {reg }}$ be the second projection from

$$
\widetilde{\mathfrak{Y}}=\left\{(\check{\boldsymbol{y}}, \boldsymbol{\alpha}) \in \check{\mathbb{T}} \times\left(\left(\mathbb{G}_{\mathrm{m}}\right)^{m}\right)^{\mathrm{reg}} \mid \check{W}_{\boldsymbol{\alpha}}(\check{\boldsymbol{y}})=1\right\} .
$$

Assume that $X$ is Fano. Any fiber $\check{Y}_{\boldsymbol{\alpha}}:=\widetilde{\varphi}^{-1}(\boldsymbol{\alpha})$ is an uncompactified mirror of a general anti-canonical hypersurface $Y \subset X$. The closure of $\check{Y}_{\boldsymbol{\alpha}}$ in $\check{X}$ is a smooth anti-canonical Calabi-Yau hypersurface, which is the compact mirror of $Y$. The quotient of the family $\widetilde{\varphi}: \widetilde{\mathfrak{Y}} \rightarrow\left(\left(\mathbb{G}_{\mathrm{m}}\right)^{m}\right)^{\text {reg }}$ by the free $\check{\mathbb{T}}$-action

$$
\check{\mathbb{T}} \ni \check{\boldsymbol{y}}:\left(\check{\boldsymbol{y}}^{\prime},\left(\alpha_{1}, \ldots, \alpha_{m}\right)\right) \mapsto\left(\check{\boldsymbol{y}}^{-1} \check{\boldsymbol{y}}^{\prime},\left(\check{\boldsymbol{y}}^{\boldsymbol{b}_{1}} \alpha_{1}, \ldots, \check{\boldsymbol{y}}^{\boldsymbol{b}_{m}} \alpha_{m}\right)\right)
$$

will be denoted by $\check{\varphi}: \check{\mathfrak{Y}} \rightarrow \check{\mathbb{L}}^{\text {reg }}$, where $\check{\mathfrak{Y}}:=\check{\mathfrak{Y}} / \check{\mathbb{T}}$ and $\check{\mathbb{L}}^{\text {reg }}:=\left(\left(\mathbb{G}_{\mathrm{m}}\right)^{m}\right)^{\mathrm{reg}} / \check{\mathbb{T}}$.

6.7. Choose an integral basis $\boldsymbol{p}_{1}, \ldots, \boldsymbol{p}_{r}$ of $\check{\boldsymbol{L}} \cong \operatorname{Pic} X$ such that each $\boldsymbol{p}_{i}$ is nef. This gives the corresponding coordinate $\boldsymbol{q}=\left(q_{1}, \ldots, q_{r}\right)$ on $\check{\mathbb{L}}$. Let $\check{U}^{\prime} \subset \check{\mathbb{L}}^{\text {reg }}$ be a neighborhood of $q_{1}=\cdots=q_{r}=0$, and $\check{U}$ be the universal cover of $\check{U}^{\prime}$.

6.8. We write the image of the Poincaré residue as

$$
H_{\text {res }}^{n-1}\left(\check{Y}_{\alpha}\right):=\operatorname{Im}\left(\operatorname{Res}: H^{0}\left(\check{X}, \Omega_{\check{X}}^{n}\left(* \check{Y}_{\alpha}\right)\right) \rightarrow H^{n-1}\left(\check{Y}_{\alpha}\right)\right) .
$$

Let $H_{\mathrm{B}}$ be the pull-back to $\check{U}$ of the local system $\operatorname{gr}_{n-1}^{W} R^{n-1} \check{\varphi}_{!} \mathbb{C}_{\check{\mathfrak{Y}}}$ on $\check{U}^{\prime}$, and $H_{\mathrm{B}}^{\text {res }}$ be the sub-system with stalks $H_{\text {res }}^{n-1}\left(\check{Y}_{\alpha}\right)$. Here $\operatorname{gr}_{n-1}^{W}$ is the weight $n-1$ piece of Deligne's mixed Hodge structure. The residual B-model VHS $\left(\mathcal{H}_{\mathrm{B}}, \nabla_{\mathrm{B}}, \mathscr{F}_{\mathrm{B}}^{\bullet}, Q_{\mathrm{B}}\right)$ on $\check{U}$ consists of the locally free sheaf $\mathcal{H}_{\mathrm{B}}:=H_{\mathrm{B}}^{\text {res }} \otimes_{\mathbb{C}} \mathcal{O}_{\check{U}}$, the Gauss-Manin connection $\nabla_{B}$, the Hodge filtration $\mathscr{F}_{B}^{\bullet}$, and the polarization $Q_{B}: \mathcal{H}_{\mathrm{B}} \otimes_{\mathcal{O}_{\check{U}}} \mathcal{H}_{\mathrm{B}} \rightarrow \mathcal{O}_{\check{U}}$ given by

$$
Q_{\mathrm{B}}\left(\omega_{1}, \omega_{2}\right):=(-1)^{(n-1)(n-2) / 2} \int_{\check{Y}_{\alpha}} \omega_{1} \cup \omega_{2} .
$$

6.9. On the A-model side, let

$$
H_{\mathrm{amb}}^{\bullet}(Y ; \mathbb{C}):=\operatorname{Im}\left(\iota^{*}: H^{\bullet}(X ; \mathbb{C}) \rightarrow H^{\bullet}(Y ; \mathbb{C})\right)
$$

be the subspace of $H^{\bullet}(Y ; \mathbb{C})$ coming from the cohomology classes of the ambient toric variety, and set

$$
U:=\left\{\boldsymbol{\tau}=\boldsymbol{\beta}+\sqrt{-1} \boldsymbol{\omega} \in H_{\mathrm{amb}}^{2}(Y ; \mathbb{C}) \mid\langle\boldsymbol{\omega}, \boldsymbol{d}\rangle \gg 0 \text { for any non-zero } \boldsymbol{d} \in \operatorname{Eff}(Y)\right\} .
$$

This open subset $U$ is considered as a neighborhood of the large radius limit point. Let $\left(\tau_{i}\right)_{i=1}^{r}$ be the coordinate on $H_{\mathrm{amb}}^{2}(Y ; \mathbb{C})$ dual to the basis $\left\{\boldsymbol{p}_{i}\right\}_{i=1}^{r}$ so that $\boldsymbol{\tau}=\sum_{i=1}^{r} \tau_{i} \boldsymbol{p}_{i}$. 
6.10. The ambient $A$-model VHS $\left(\mathcal{H}_{\mathrm{A}}, \nabla_{\mathrm{A}}, \mathscr{F}_{\mathrm{A}}^{\bullet}, Q_{\mathrm{A}}\right)$ consists ([Iri11, Definition 6.2], cf. also [CK99, Section 8.5]) of the locally free sheaf $\mathcal{H}_{\mathrm{A}}=H_{\mathrm{amb}}^{\bullet}(Y ; \mathbb{C}) \otimes_{\mathbb{C}} \mathcal{O}_{U}$, the connection

$$
\nabla_{\mathrm{A}}=d+\sum_{i=1}^{r}\left(\boldsymbol{p}_{i} \circ_{\boldsymbol{\tau}}\right) d \tau_{i}: \mathcal{H}_{\mathrm{A}} \rightarrow \mathcal{H}_{\mathrm{A}} \otimes \Omega_{U}^{1}
$$

the Hodge filtration

$$
\mathscr{F}_{\mathrm{A}}^{p}:=H_{\mathrm{amb}}^{\leq 2(n-1-p)}(Y ; \mathbb{C}) \otimes_{\mathbb{C}} \mathcal{O}_{U}
$$

and the pairing

$$
Q_{A}: \mathcal{H}_{\mathrm{A}} \otimes_{\mathcal{O}_{U}} \mathcal{H}_{\mathrm{A}} \rightarrow \mathcal{O}_{U}, \quad(\alpha, \beta) \mapsto(2 \pi \sqrt{-1})^{n-1} \int_{Y}(-1)^{\operatorname{deg} \alpha / 2} \alpha \cup \beta,
$$

which is $(-1)^{n-1}$-symmetric and $\nabla_{\mathrm{A}}$-flat.

6.11. Let $\boldsymbol{u}_{i} \in H_{\mathrm{amb}}^{2}(Y ; \mathbb{Z})$ be the first Chern class of the line bundle on $Y$ corresponding to the one-dimensional cone $\mathbb{R}_{\geq 0} \cdot \boldsymbol{b}_{i} \in \Sigma$ and $\boldsymbol{v}=\boldsymbol{u}_{1}+\cdots+\boldsymbol{u}_{m}$ be the restriction of the anti-canonical class of $X$. Denote $\boldsymbol{t}:=\sum_{i=1}^{r} t_{i} \boldsymbol{p}_{i}$. Givental's $I$-function is defined as the series

$$
I_{Y}(\boldsymbol{t} ; \mathbf{z})=e^{\boldsymbol{t} / \mathbf{z}} \sum_{\boldsymbol{d} \in \operatorname{Eff}(X)} e^{\boldsymbol{d} \cdot \boldsymbol{t}} \frac{\prod_{k=-\infty}^{\langle\boldsymbol{d}, \boldsymbol{v}\rangle}(\boldsymbol{v}+k \mathbf{z}) \prod_{j=1}^{m} \prod_{k=-\infty}^{0}\left(\boldsymbol{u}_{j}+k \mathbf{z}\right)}{\prod_{k=-\infty}^{0}(\boldsymbol{v}+k \mathbf{z}) \prod_{j=1}^{m} \prod_{k=-\infty}^{\left\langle\boldsymbol{d}, \boldsymbol{u}_{j}\right\rangle}\left(\boldsymbol{u}_{j}+k \mathbf{z}\right)},
$$

which is a multi-valued map from $\check{U^{\prime}}$ (or a single-valued map from $\check{U}$ ) to the classical cohomology group $H_{\mathrm{amb}}^{\bullet}\left(Y ; \mathbb{C}\left[\mathrm{z}^{-1}\right]\right)$. The $J$-function is defined by

$$
J_{Y}(\boldsymbol{\tau} ; \mathbf{z})=L_{Y}(\boldsymbol{\tau}, \mathbf{z})^{-1}(1),
$$

where $L_{Y}(\boldsymbol{\tau}, \mathbf{z})$ is the fundamental solution of the quantum differential equation defined explicitly by using the Gromov-Witten invariants as in [Iri11, Equation (2.3)] with c set to 1 . If we write

$$
I_{Y}(\boldsymbol{t} ; \mathrm{z})=F(\boldsymbol{t}) \mathbf{1}+\frac{G(\boldsymbol{t})}{\mathrm{z}}+O\left(\mathrm{z}^{-2}\right),
$$

then Givental's mirror theorem [Giv98] states that

$$
I_{Y}(\boldsymbol{t} ; \mathbf{z})=F(\boldsymbol{t}) \cdot J_{Y}(\boldsymbol{\varsigma}(\boldsymbol{t}) ; \mathbf{z}),
$$

where the mirror map $\varsigma: \check{U} \rightarrow H_{\mathrm{amb}}^{2}(Y ; \mathbb{C})$ is defined by

$$
\boldsymbol{\varsigma}(\boldsymbol{t})=\iota^{*}\left(\frac{G(\boldsymbol{t})}{F(\boldsymbol{t})}\right) \text {. }
$$

The relation between $\boldsymbol{\tau}=\boldsymbol{\varsigma}(\boldsymbol{t})$ and $\boldsymbol{\sigma}=\boldsymbol{\beta}+\sqrt{-1} \boldsymbol{\omega}$ is given by $\boldsymbol{\tau}=2 \pi \sqrt{-1} \boldsymbol{\sigma}$, so that $\mathfrak{I m}(\boldsymbol{\sigma}) \gg 0$ corresponds to $\exp (\boldsymbol{\tau}) \sim 0$. The functions $F(\boldsymbol{t})$ and $G(\boldsymbol{t})$ satisfy the Picard-Fuchs equations, and give periods for the B-model VHS $\left(\mathcal{H}_{\mathrm{B}}, \nabla^{B}, \mathscr{F}_{B}^{\bullet}, Q_{B}\right)$.

6.12. (6.11.4) implies the existence of an isomorphism

$$
\operatorname{Mir}_{\mathcal{Y}}: \varsigma^{*}\left(\mathcal{H}_{\mathrm{A}}, \nabla_{\mathrm{A}}, \mathscr{F}_{\mathrm{A}}^{\bullet}, Q_{A}\right) \stackrel{\sim}{\rightarrow}\left(\mathcal{H}_{\mathrm{B}}, \nabla_{\mathrm{B}}, \mathscr{F}_{\mathrm{B}}^{\bullet}, Q_{B}\right)
$$

of variations of polarized Hodge structures, which sends $F(\boldsymbol{t}) \mathbf{1}$ on the left-hand side to

$$
\Omega:=\operatorname{Res}\left(\frac{1}{\check{f}_{\boldsymbol{\alpha}}} \frac{d \check{y}_{1}}{\check{y}_{1}} \wedge \cdots \wedge \frac{d \check{y}_{n}}{\check{y}_{n}}\right)
$$


on the right-hand side. A stronger statement, which gives an isomorphism of the $\widehat{\Gamma}$ integral structure on the A-side and the natural integral structure on the B-side, is proved in [Iri11, Theorem 6.9].

\section{QUASIMAP CORRELATION FUNCTIONS FOR ANTI-CANONICAL HYPERSURFACES IN} TORIC VARIETIES

7.1. For $\boldsymbol{d} \in \operatorname{Eff}(X)$ and $i \in\{1, \ldots, m\}$, we set

$$
k_{i}:= \begin{cases}\left\langle\boldsymbol{u}_{i}, \boldsymbol{d}\right\rangle & \left\langle\boldsymbol{u}_{i}, \boldsymbol{d}\right\rangle \geq 0, \\ -1 & \left\langle\boldsymbol{u}_{i}, \boldsymbol{d}\right\rangle<0 .\end{cases}
$$

and define the quasimap space of degree $\boldsymbol{d}$ by

$$
\mathbf{Q}(X ; \boldsymbol{d}):=\left(\prod_{i=1}^{m} \mathbb{A}_{i}^{k_{i}+1}\right) / /{ }_{\theta} \mathbb{L}
$$

with (6.3.7) in mind. An argument parallel to that in $\operatorname{Section~} 3.1$ shows that $\mathbf{Q}(X ; \boldsymbol{d})$ is a compactification of the space of holomorphic maps $\mathbb{P}^{1} \rightarrow X$ of degree $\boldsymbol{d}$. Later in Section 10.4, we will introduce the moduli spaces $\mathbf{Q}(W / / G ; \boldsymbol{d})$ of degree $\boldsymbol{d}$ quasimaps from $\mathbb{P}^{1}$ to more general GIT quotients $W / / G$, and $\mathbf{Q}(X ; \boldsymbol{d})$ here is the special case for $W=\prod_{i=1}^{m} \mathbb{A}_{i}^{k_{i}+1}$ and $G=\mathbb{L}$. The first Chern class of the line bundle on $\mathbf{Q}(X ; \boldsymbol{d})$ associated with the character $\chi_{i}$ of $\mathbb{L}$ will also be denoted by $\boldsymbol{u}_{i}$ by abuse of notation. The Morrison-Plesser class is defined by

$$
\Phi_{\boldsymbol{d}}:=\left(\boldsymbol{u}_{1}+\cdots+\boldsymbol{u}_{m}\right)^{\left\langle\boldsymbol{u}_{1}+\cdots+\boldsymbol{u}_{m}, \boldsymbol{d}\right\rangle} \prod_{\left\langle\boldsymbol{u}_{i}, \boldsymbol{d}\right\rangle<0} \boldsymbol{u}_{i}^{-\left\langle\boldsymbol{u}_{i}, \boldsymbol{d}\right\rangle-1} .
$$

Here, the latter part $\prod_{\left\langle\boldsymbol{u}_{i}, \boldsymbol{d}\right\rangle<0} \boldsymbol{u}_{i}^{-\left\langle\boldsymbol{u}_{i}, \boldsymbol{d}\right\rangle-1}$ is the Euler class of $h^{1}\left(E^{\vee}\right)$ where $E$ is the canonical obstruction theory for $\mathbf{Q}(X ; \boldsymbol{d})$ defined in Section 10.7. The first part $\left(\boldsymbol{u}_{1}+\right.$ $\left.\cdots+\boldsymbol{u}_{m}\right)^{\left\langle\boldsymbol{u}_{1}+\cdots+\boldsymbol{u}_{m}, \boldsymbol{d}\right\rangle}$ is the Euler class of the vector bundle formed by the obstruction spaces to being a quasimap to an anti-canonical hypersurface $Y \subset X$ for each element in $\mathbf{Q}(X ; \boldsymbol{d})$. For a polynomial $P\left(\alpha_{1}, \ldots, \alpha_{m}\right) \in \mathbb{C}\left[\alpha_{1}, \ldots, \alpha_{m}\right]$, we set

$$
\left\langle P\left(\boldsymbol{u}_{1}, \ldots, \boldsymbol{u}_{m}\right)\right\rangle_{X, Y, \boldsymbol{d}}:=\int_{\mathbf{Q}(X ; \boldsymbol{d})} P\left(\boldsymbol{u}_{1}, \ldots, \boldsymbol{u}_{m}\right) \Phi_{\boldsymbol{d}}
$$

and

$$
\left\langle P\left(\boldsymbol{u}_{1}, \ldots, \boldsymbol{u}_{m}\right)\right\rangle_{X, Y}:=\sum_{\boldsymbol{d} \in \operatorname{Eff}(X)} \boldsymbol{\alpha}^{\boldsymbol{d}}\left\langle P\left(\boldsymbol{u}_{1}, \ldots, \boldsymbol{u}_{m}\right)\right\rangle_{X, Y, \boldsymbol{d}} \in \mathbb{Z} \llbracket \alpha^{\boldsymbol{d}}: \boldsymbol{d} \in \operatorname{Eff}(X) \rrbracket
$$

where the completion is taken with respect to the ideal generated by $\operatorname{Eff}(X) \backslash\{0\}$. Here $\boldsymbol{\alpha}^{\boldsymbol{d}}$ is defined by (6.3.4).

\section{TORIC RESIDUE MIRROR SYMMETRY}

8.1. Let $\check{G}=\left(\check{G}_{0}, \ldots, \check{G}_{n}\right)$ be a regular sequence in $S_{\check{\Delta}}$. If we set $I_{\check{G}}:=I_{\check{\Delta}} /\left(\check{G}_{0}, \ldots, \check{G}_{n}\right) I_{\check{\Delta}}$, then the graded piece $I_{\breve{G}}^{n+1}$ is one-dimensional and spanned by $J_{\breve{G}}:=\operatorname{det}\left(\check{y}_{i} \partial_{\check{y}_{i}} \check{G}_{j}\right)_{i, j=0}^{n}$. The toric residue [Cox96] is the map $\operatorname{Res}_{\breve{G}}: I_{\check{\Delta}}^{n+1} \rightarrow \mathbb{C}$ sending $\left(\check{G}_{0}, \ldots, \check{G}_{n}\right) I_{\check{\Delta}}$ to zero and $J_{\breve{G}}$ to the normalized volume $\operatorname{vol}(\check{\Delta})$, i.e., $n$ ! times the Euclidean volume of $\check{\Delta}$. For $\boldsymbol{\alpha} \in \check{\mathbb{L}}^{\text {reg }}$, we define $\check{F}_{\boldsymbol{\alpha}}$ as in (6.5.2) and write $\operatorname{Res}_{\check{f}_{\boldsymbol{\alpha}}}:=\operatorname{Res}_{\breve{F}_{\boldsymbol{\alpha}}}$. Theorem 8.2 below is introduced in [BM02, Conjecture 4.6] and proved in [SV04, Bor05]. 
Theorem 8.2. For any homogeneous polynomial $P\left(\alpha_{1}, \ldots, \alpha_{m}\right) \in \mathbb{C}\left[\alpha_{1}, \ldots, \alpha_{m}\right]$ of degree $n$, the generating function (7.1.5) gives the Laurent expansion of the toric residue

$$
\left\langle P\left(\boldsymbol{u}_{1}, \ldots, \boldsymbol{u}_{m}\right)\right\rangle_{X, Y}=(-1)^{n} \operatorname{Res}_{\check{f}_{\boldsymbol{\alpha}}}\left(\check{y}_{0}^{n+1} P\left(\alpha_{1} \check{\boldsymbol{y}}^{\boldsymbol{b}_{1}}, \ldots, \alpha_{m} \check{\boldsymbol{y}}^{\boldsymbol{b}_{m}}\right)\right)
$$

around the large radius limit point associated with the fan $\Sigma$.

[BM02, Conjecture 4.6] is generalized to toric complete intersections in [BM03, Conjecture 4.6] and proved in [Kar05, SV06].

8.3. The family $\varphi: \check{\mathcal{Y}} \rightarrow \check{\mathbb{L}}^{\text {reg }}$ of Calabi-Yau manifolds comes with the holomorphic volume form

$$
\Omega:=\operatorname{Res}\left(\frac{1}{\check{f}_{\alpha}} \frac{d \check{y}_{1}}{\check{y}_{1}} \wedge \cdots \wedge \frac{d \check{y}_{n}}{\check{y}_{n}}\right) \in H^{0}\left(\mathcal{H}_{\mathrm{B}}\right) .
$$

For a homogeneous polynomial $Q\left(\alpha_{1}, \ldots, \alpha_{m}\right) \in \mathbb{Q}\left[\alpha_{1}, \ldots, \alpha_{m}\right]$ of degree $n-1$, the $Q$ Yukawa $(n-1)$-point function is defined in [BM02, Definition 9.1] by

$$
Y_{Q}(\boldsymbol{\alpha}):=(-1)^{(n-1)(n-2) / 2} \frac{1}{(2 \pi \sqrt{-1})^{n-1}} \int_{\check{Y}_{\boldsymbol{\alpha}}} \Omega \wedge Q\left(\alpha_{1} \frac{\partial}{\partial \alpha_{1}}, \ldots, \alpha_{m} \frac{\partial}{\partial \alpha_{m}}\right) \Omega,
$$

where the differential operators $\alpha_{1} \partial / \partial \alpha_{1}, \ldots, \alpha_{m} \partial / \partial \alpha_{m}$ act on $\mathcal{H}_{\mathrm{B}}$ by the Gauss-Manin connection.

8.4. For $Q\left(\alpha_{1}, \ldots, \alpha_{m}\right) \in \mathbb{Q}\left[\alpha_{1}, \ldots, \alpha_{m}\right]$, we set

$$
P\left(\alpha_{1}, \ldots, \alpha_{m}\right):=\left(\alpha_{1}+\cdots+\alpha_{m}\right) Q\left(\alpha_{1}, \ldots, \alpha_{m}\right) \in \mathbb{Q}\left[\alpha_{1}, \ldots, \alpha_{m}\right] .
$$

By [BM02, Theorem 9.7], which is attributed to [Mav00, one has an equality

$$
Y_{Q}(\boldsymbol{\alpha})=(-1)^{n} \operatorname{Res}_{\check{f}_{\boldsymbol{\alpha}}}\left(\check{y}_{0}^{n} P\left(\alpha_{1} \check{\boldsymbol{y}}^{\boldsymbol{b}_{1}}, \ldots, \alpha_{m} \check{\boldsymbol{y}}^{\boldsymbol{b}_{m}}\right)\right)
$$

of the Yukawa $(n-1)$-point function and the toric residue.

8.5. Assume that the unstable locus of the $\mathbb{L}$-action on $\mathbb{A}^{m}$ with respect to $\theta$ has codimension strictly greater than 1 . Then one has $H^{2}\left(X_{\Sigma}\right)=\operatorname{Pic}\left(X_{\Sigma}\right)=\operatorname{Pic}^{\mathbb{L}}\left(\mathbb{A}^{m}\right)$ so that the class $\boldsymbol{p}_{i}$ corresponds to a one-dimensional representation $\mathbb{C}_{\boldsymbol{p}_{i}}$ of $\mathbb{L}$. By abuse of notation, we let $\boldsymbol{p}_{i}$ denote the $\mathbb{G}_{\mathrm{m}}$-equivariant Euler class of the pull-back of the line bundle $\left[\mathbb{A}^{m} \times \mathbb{C}_{\boldsymbol{p}_{i}} / \mathbb{G}_{\mathrm{m}}\right]$ by the evaluation map ev $0: X_{\boldsymbol{d}} \rightarrow\left[\mathbb{A}^{m} / \mathbb{G}_{\mathrm{m}}\right]$ at $0 \in \mathbb{P}^{1}$. Denote $\boldsymbol{v}:=\sum_{i=1}^{m} \boldsymbol{u}_{i}$.

If we set

$$
\Phi(\boldsymbol{t}, \boldsymbol{\tau} ; \mathbf{z}):=\sum_{\boldsymbol{d} \in \operatorname{Eff}(X)} e^{\boldsymbol{\tau} \cdot \boldsymbol{d}} \int_{\mathbf{Q}(X ; \boldsymbol{d})}^{\mathbb{G}_{\mathrm{m}}} e^{(\boldsymbol{t}-\boldsymbol{\tau}) / \mathrm{z}} \Phi_{\boldsymbol{d}} \boldsymbol{v}
$$

then for any polynomial $R\left(t_{1}, \ldots, t_{r}\right) \in \mathbb{Q}\left[t_{1}, \ldots, t_{r}\right]$, one has

$$
\left.R\left(\mathrm{z} \frac{\partial}{\partial t_{1}}, \ldots, \mathrm{z} \frac{\partial}{\partial t_{r}}\right) \Phi(\boldsymbol{t}, \boldsymbol{\tau} ; \mathrm{z})\right|_{\boldsymbol{\tau}=\boldsymbol{t}}=\sum_{\boldsymbol{d} \in \operatorname{Eff}(X)} e^{\boldsymbol{t} \cdot \boldsymbol{d}} \int_{\mathbf{Q}(X ; \boldsymbol{d})}^{\mathbb{G}_{\mathrm{m}}} R\left(\boldsymbol{p}_{1}, \ldots, \boldsymbol{p}_{r}\right) \Phi_{\boldsymbol{d}} \boldsymbol{v}
$$

In addition, one has

$$
\Phi(\boldsymbol{t}, \boldsymbol{\tau} ; \mathbf{z})=\int_{Y} I(\boldsymbol{t} ;-\mathbf{z}) \cup I(\boldsymbol{\tau} ; \mathbf{z}),
$$

by [Giv98, Proposition 6.2]. This is the toric hypersurface version of (3.12.2). Note that $I(\boldsymbol{t} ; 1)$ is convergent for large enough $-\operatorname{Re} \boldsymbol{t}$ by ratio test on the series (6.11.1) without the prefactor. By specializing to $\mathbf{z}=1$ and using the definition of $Q_{\mathrm{A}}$, one obtains

$$
\Phi(\boldsymbol{t}, \boldsymbol{\tau} ; 1)=Q_{\mathrm{A}}(I(\boldsymbol{t} ; 1), I(\boldsymbol{\tau} ; 1)) .
$$


By combining (8.5.4) with (6.11.4), one obtains

$$
\Phi(\boldsymbol{t}, \boldsymbol{\tau} ; 1)=Q_{\mathrm{A}}\left(L^{-1}(\boldsymbol{t} ; 1) F(\boldsymbol{t}) \mathbf{1}, L^{-1}(\boldsymbol{\tau} ; 1) F(\boldsymbol{\tau}) \mathbf{1}\right) .
$$

Since $L$ is the fundamental solution for the flat connection $\nabla_{\mathrm{B}}$, the function $\Phi(\boldsymbol{t}, \boldsymbol{\tau} ; 1)$ is obtained by parallel-transporting $F(\boldsymbol{t}) \mathbf{1} \in\left(H_{\mathrm{B}}\right)_{\boldsymbol{t}}$ and $F(\boldsymbol{\tau}) \mathbf{1} \in\left(H_{\mathrm{B}}\right)_{\boldsymbol{\tau}}$ to the fiber at the same point and taking the pairing $Q_{\mathrm{B}}$ at that point (the result does not depend on the choice of the point since $Q_{\mathrm{B}}$ is $\nabla_{\mathrm{B}}$-parallel). By sending (2.5.5) by (6.12.1), one obtains

$$
(2 \pi \sqrt{-1})^{n-1} \int_{Y} I(\boldsymbol{t} ;-1) I(\boldsymbol{\tau} ; 1)=(-1)^{(n-1)(n-2) / 2} \int_{\check{Y}} \Omega_{\boldsymbol{t}} \wedge \Omega_{\boldsymbol{\tau}} .
$$

Assume that $P\left(\alpha_{1}, \ldots, \alpha_{m}\right)=\left(\alpha_{1}+\cdots+\alpha_{m}\right) Q\left(\alpha_{1}, \ldots, \alpha_{m}\right)$ for a polynomial $Q$ and take $\left.R\left(t_{1}, \ldots, t_{r}\right):=Q\left(\sum_{i=1}^{r} a_{i, 1} t_{i}, \ldots, \sum_{i=1}^{r} a_{i, m} t_{i}\right)\right)$ where $a_{i, j}$ are integers uniquely satisfying $\chi_{j}=\sum_{i=1}^{r} a_{i, j} \boldsymbol{p}_{i}$. By differentiating (8.5.6) by $R\left(\partial_{t_{1}}, \ldots, \partial_{t_{r}}\right.$ ) and setting $\boldsymbol{\tau}=\boldsymbol{t}$, we obtain toric residue mirror symmetry for polynomials of the form $P\left(\alpha_{1}, \ldots, \alpha_{m}\right)=\left(\alpha_{1}+\right.$ $\left.\cdots+\alpha_{m}\right) Q\left(\alpha_{1}, \ldots, \alpha_{m}\right)$.

\section{MARTIN'S FORMULA}

9.1. We use the same notations $G, T, \mathscr{W}$, and $\Delta$ for a reductive algebraic group, a maximal torus, the Weyl group, and the set of roots as in Section 2, Let $W$ be an affine scheme with $G$-action, and fix a character $\theta$ of $G$. We write the line bundle on $W / / T$ associated with $\alpha \in \Delta$ as $L_{\alpha}$, and set

$$
e:=\prod_{\alpha \in \Delta} c_{1}\left(L_{\alpha}\right) \in H^{2|\Delta|}(W / / T ; \mathbb{Z}) .
$$

We write the natural projection and inclusion as and say that $\widetilde{a} \in H^{*}(W / / T)$ is a lift of $a \in H^{*}(W / / G)$ if $\pi^{*} a=\iota^{*} \widetilde{a}$.

Theorem 9.2 (Martin $[\mathrm{Mar}]$ ). If $\widetilde{a}$ is a lift of $a$, then one has

$$
\int_{W / / G} a=\frac{1}{|\mathscr{W}|} \int_{W / T} \widetilde{a} \cup e .
$$

9.3. Let $\operatorname{Mat}(r, n) \cong \mathbb{A}^{r \times n}$ be the space of $n \times r$ matrices, which is considered as the space of linear maps from an $r$-dimensional vector space to an $n$-dimensional vector space. It has a natural action of $\mathrm{GL}_{r}$, and the GIT quotient $\operatorname{Gr}(r, n):=\operatorname{Mat}(r, n) / / \mathrm{GL}_{r}$ is the Grassmannian of $r$-spaces in an $n$-space.

9.4. When $W=\operatorname{Mat}(r, n)$ and $G=\mathrm{GL}_{r}$, one has

$$
\begin{aligned}
& W / / G \cong \operatorname{Gr}(r, n), \\
& W / / T \cong\left(\mathbb{P}^{n-1}\right)^{r}
\end{aligned}
$$

and

$$
\begin{aligned}
H^{*}(\operatorname{Gr}(r, n)) & \cong \mathbb{C}\left[\sigma_{1}, \ldots, \sigma_{r}\right] /\left(h_{n-r+1}, \ldots, h_{n}\right), \\
H^{*}\left(\left(\mathbb{P}^{n-1}\right)^{r}\right) & \cong \mathbb{C}\left[x_{1}, \ldots, x_{r}\right] /\left(x_{1}^{n}, \ldots, x_{r}^{n}\right),
\end{aligned}
$$

where $\sigma_{i}=\sigma_{i}\left(x_{1}, \ldots, x_{r}\right) \in \mathbb{C}\left[x_{1}, \ldots, x_{r}\right]^{\mathfrak{S}_{r}}$ are elementary symmetric functions and $h_{i}=h_{i}\left(x_{1}, \ldots, x_{r}\right) \in \mathbb{C}\left[x_{1}, \ldots, x_{r}\right]^{\mathfrak{S}_{r}}=\mathbb{C}\left[\sigma_{1}, \ldots, \sigma_{r}\right]$ are complete symmetric functions. 
Martin's formula in this case gives

$$
\begin{aligned}
\int_{\operatorname{Gr}(r, n)} P\left(x_{1}, \ldots, x_{r}\right) & =\frac{1}{r !} \int_{\left(\mathbb{P}^{n-1}\right)^{r}} \prod_{i \neq j}\left(x_{i}-x_{j}\right) P\left(x_{1}, \ldots, x_{r}\right) \\
& =\frac{(-1)^{r(r-1) / 2}}{r !} \int_{\left(\mathbb{P}^{n-1}\right)^{r}} \Delta^{2} \cup P\left(x_{1}, \ldots, x_{r}\right)
\end{aligned}
$$

for any $P\left(x_{1}, \ldots, x_{r}\right) \in \mathbb{C}\left[x_{1}, \ldots, x_{r}\right]^{\mathfrak{S}_{r}}$ where $\boldsymbol{\Delta}:=\prod_{1 \leq i<j \leq r}\left(x_{i}-x_{j}\right)$.

9.5. The equivariant cohomology ring of $\operatorname{Gr}(r, n)$ with respect to the natural action of the diagonal maximal abelian subgroup $H \subset \mathrm{GL}_{n}$ is presented as

$$
H_{H}^{\bullet}(\mathrm{Gr}(r, n) ; \mathbb{C}) \cong \mathbb{C}\left[\sigma_{1}, \ldots, \sigma_{r}, \lambda_{1}, \ldots, \lambda_{n}\right] /\left(h_{n-r+1}(\sigma, \lambda), \ldots, h_{n}(\sigma, \lambda)\right),
$$

where $h_{i}$ is the degree $2 i$ part of $c^{H}(\mathcal{S}) c^{H}(\mathcal{Q})-\prod_{i=1}^{n}\left(1+\lambda_{i}\right)$. Here $\mathcal{S}$ and $\mathcal{Q}$ are the tautological bundle and the universal quotient bundle respectively, and $c^{H}(-)$ stands for the $H$-equivariant total Chern class. Note that $\sigma_{i}:=c_{i}^{H}(\mathcal{S})$ is the elementary symmetric function of the $H$-equivariant Chern $\operatorname{roots} x_{1}, \ldots, x_{r}$ of $\mathcal{S}$, and $c_{i}^{H}(\mathcal{Q})$ for $i=1, \ldots, n-r$ are expressed in terms of $\sigma_{1}, \ldots, \sigma_{r}$ and $\lambda_{1}, \ldots, \lambda_{n}$ by the condition $h_{1}=\cdots=h_{n-r}=0$. Martin's formula gives

$$
\begin{aligned}
& \int_{\operatorname{Gr}(r, n)}^{H} P\left(\sigma_{1}, \ldots, \sigma_{r}\right) \\
& \quad=\sum_{1 \leq i_{1}<i_{2}<\cdots i_{r} \leq n} \operatorname{Res}_{\boldsymbol{x}=\left(\lambda_{i_{1}}, \ldots, \lambda_{i_{r}}\right)} P\left(\sigma_{1}, \ldots, \sigma_{r}\right) \prod_{i \neq j}\left(x_{i}-x_{j}\right) \frac{d x_{1} \wedge \cdots \wedge d x_{r}}{\prod_{i=1}^{r} \prod_{j=1}^{n}\left(x_{i}-\lambda_{j}\right)}
\end{aligned}
$$

\section{Quasimap SPACES FOR GIT QUOTIEntS}

10.1. Let $G$ be a reductive algebraic group acting on an affine variety $W$ and fix a character $\theta$ of $G$. In this paper, we will always assume the following:

(1) Semi-stability implies stability.

(2) The semi-stable locus $W^{\text {ss }}$ is smooth and non-empty.

(3) The $G$-action on $W^{\text {ss }}$ is free (however, see CCFK15] for allowing finite non-trivial stablizers).

(4) The codimension of the unstable locus $W \backslash W^{\text {ss }}$ is greater than one.

The GIT quotient is defined by $W / / G:=W^{\text {ss }} / G$, which is an open substack of $[W / G]$.

10.2. A map $u: \mathbb{P}^{1} \rightarrow[W / G]$ to the quotient stack $[W / G]$ is pair $(P, \widetilde{u})$ of a principal $G$-bundle $P \rightarrow \mathbb{P}^{1}$ and a $G$-equivariant map $\widetilde{u}: P \rightarrow W$. It is called a quasimap if the generic point of $\mathbb{P}^{1}$ is mapped to $W / / G \subset[W / G]$. A point in the inverse image of the unstable locus will be called a base point.

10.3. For a quasimap $u: \mathbb{P}^{1} \rightarrow[W / G]$ and a $G$-equivariant line bundle $L$ on $W$, the pull-back $\widetilde{u}^{*} L$ is a $G$-equivariant line bundle on $P$, which descends to a line bundle $u^{*} L$ on $\mathbb{P}^{1}$. The degree of a quasimap $u: \mathbb{P}^{1} \rightarrow[W / G]$ is the map $\boldsymbol{d}: \operatorname{Pic}^{G} W \rightarrow \mathbb{Z}$ sending $L \in \operatorname{Pic}^{G} W$ to $\operatorname{deg} u^{*} L$. 
10.4. An isomorphism of quasimaps $u=(P, \widetilde{u})$ and $u^{\prime}=\left(P^{\prime}, \widetilde{u}^{\prime}\right)$ is an isomorphism $\varphi: P \rightarrow P^{\prime}$ of principal $G$-bundles such that $\widetilde{u}=\widetilde{u}^{\prime} \circ \varphi$. By [CFKM14, Theorem 7.1.6], the moduli functor for quasimaps of degree $\boldsymbol{d}$ is representable by a Deligne-Mumford stack, which will be denoted by $\mathbf{Q}(W / / G ; \boldsymbol{d})$. This stack is denoted by $\operatorname{Qmap}_{0,0}\left(W / / G, \boldsymbol{d} ; \mathbb{P}^{1}\right)$ in CFKM14, $\$ 7.2]$ and $\mathrm{QG}_{0,0, \boldsymbol{d}}^{0+}(W / / G)$ in [CFK14, Section 2.6]. Note that $\mathbf{Q}(W / / G)$ depends not only on $W / / G$ and $\boldsymbol{d}$ but also on $W, G$, and $\theta$.

10.5. Let Q. $(W / / G ; \boldsymbol{d}) \subset \mathbf{Q}(W / / G ; \boldsymbol{d})$ be the substack parametrizing quasimaps such that $\left.u\right|_{\mathbb{P}^{1} \backslash\{0\}}$ is a constant map to $W / / G$. This implies that $0 \in \mathbb{P}^{1}$ is a base point of length $\boldsymbol{d}(\theta)$ by [CFKM14, Lemma 7.1.2]. This stack is denoted by $\mathbf{Q}_{0,0+}(W / / G, \boldsymbol{d})_{0}$ in CFK14, Section 4.1]. There is a natural map ev: Q. $(W / / G ; \boldsymbol{d}) \rightarrow W / / G$, called the evaluation map, which sends $u \in \mathbf{Q} .(W / / G ; \boldsymbol{d})$ to $u(\infty) \in W / / G$.

10.6. There is a natural $\mathbb{G}_{\mathrm{m}}$-action on $\mathbf{Q}(W / / G ; \boldsymbol{d})$ coming from the standard $\mathbb{G}_{\mathrm{m}}$-action on $\mathbb{P}^{1}$. As described in [CFK14, Section 4.1], the fixed locus of this action is identified with the coproduct

$$
\coprod_{\boldsymbol{d}_{1}+\boldsymbol{d}_{2}=\boldsymbol{d}} \text { Q. }\left(W / / G ; \boldsymbol{d}_{1}\right) \times_{W / / G} \mathbf{Q} \cdot\left(W / / G ; \boldsymbol{d}_{2}\right)
$$

of fiber products with respect to the evaluation map.

10.7. If $W$ has at worst lci singularity, then $\mathbf{Q}(W / / G ; \boldsymbol{d})$ has a canonical perfect obstruction theory, which allows one to define the virtual fundamental cycle. The canonical perfect obstruction theory is $\left(\mathbb{R} \pi_{*} \mathrm{ev}^{*} T_{[W / G]}\right)^{\vee}$, where $T_{[W / G]}$ is the tangent complex of $[W / G]$, ev : $\mathbf{Q}(W / / G ; \boldsymbol{d}) \times \mathbb{P}^{1} \rightarrow[W / G]$ is the evaluation map, and $\pi: \mathbf{Q}(W / / G ; \boldsymbol{d}) \times \mathbb{P}^{1} \rightarrow$ $\mathbf{Q}(W / / G ; \boldsymbol{d})$ is the first projection; see Theorem 7.2.2 of [CFK14] or Section 5. The virtual fundamental cycle is an element of the Chow group of $\mathbf{Q}(W / / G ; \boldsymbol{d})$ whose degree is given by the virtual dimension

$$
\text { virt.dim } \mathbf{Q}(W / / G ; \boldsymbol{d})=\left\langle\boldsymbol{d}, \operatorname{det} T_{W}\right\rangle+\operatorname{dim} W / / G .
$$

10.8. Since the stack Q. $(W / / G ; \boldsymbol{d})$ is the union of connected components of the fixed locus of the $\mathbb{G}_{\mathrm{m}}$-action, it has a perfect obstruction theory inherited from $\mathbf{Q}(W / / G ; \boldsymbol{d})$. The virtual push-forward

$$
\mathrm{ev}_{*}^{\mathrm{virt}}(-):=\operatorname{PD}\left(\mathrm{ev}_{*}\left((-) \cap[\mathbf{Q} .(W / / G ; \boldsymbol{d})]^{\mathrm{virt}}\right)\right)
$$

along the evaluation map ev: Q. $(W / / G ; \boldsymbol{d}) \rightarrow W / / G$ allows one to define the $I$-function

$$
I(\boldsymbol{t} ; \mathbf{z}):=e^{\boldsymbol{p} \cdot \boldsymbol{t} / \mathbf{z}} \sum_{\boldsymbol{d} \in \operatorname{Eff}(W / / G)} e^{\boldsymbol{d} \cdot \boldsymbol{t}} I_{\boldsymbol{d}}
$$

by

$$
\left.I_{\boldsymbol{d}}:=\operatorname{ev}_{*}^{\operatorname{virt}}\left(\frac{1}{\operatorname{Eul}^{\mathbb{G}_{\mathrm{m}}}\left(N_{\mathbf{Q}}^{\mathrm{virt}}(W / / G ; \boldsymbol{d}) / \mathbf{Q}(W / / G ; \boldsymbol{d})\right.}\right)\right),
$$

where the denominator is the $\mathbb{G}_{\mathrm{m}}$-equivariant Euler class of the virtual normal bundle. Here $\boldsymbol{p}$ is a basis of $H^{2}(W / / G)$, and $\boldsymbol{t}$ is the coordinate of $H^{2}(W / / G)$ corresponding to $\boldsymbol{p}$.

An $H$-action on $W$ commuting with the $G$-action induces an $H$-action on Q. $(W / / G ; \boldsymbol{d})$, which allows one to define the $H$-equivariant $I$-function of $W / / G$. 
10.9. There exits a $G$-space $V$ with a $G$-equivariant closed embedding $W \hookrightarrow V$. Let $\mathfrak{u}: \mathbf{Q}(W / / G ; \boldsymbol{d}) \times \mathbb{P}^{1} \rightarrow[W / G]$ be the universal quasimap. It consists of a principal $G$ bundle $\mathcal{P}$ on $\mathbf{Q}(W / / G ; \boldsymbol{d}) \times \mathbb{P}^{1}$ and a $G$-equivariant morphism $\tilde{\mathfrak{u}}: \mathcal{P} \rightarrow W$. Let $\mathcal{P}^{\prime}:=$ $\left.\mathcal{P}\right|_{\mathbf{Q}(W / / G ; \boldsymbol{d}) \times\{\mathrm{pt}\}}$ be the restriction of $\mathcal{P}$ to a fiber of the second projection $\mathbf{Q}(W / / G ; \boldsymbol{d}) \times$ $\mathbb{P}^{1} \rightarrow \mathbb{P}^{1}$. We write the Chern-Weil homomorphism defined by $\mathcal{P}^{\prime}$ as

$$
\mathfrak{C W}: \mathbb{C}[\mathfrak{g}]^{G} \rightarrow H^{*}(\mathbf{Q}(W / / G ; \boldsymbol{d})) .
$$

Note that $\mathbb{C}[\mathfrak{g}]^{G}$ is isomorphic to $\mathbb{C}[\mathfrak{t}]^{\mathscr{W}}$ by Chevalley restriction theorem. For $P \in \mathbb{C}[\mathfrak{t}]^{\mathscr{W}}$, we set

$$
\begin{aligned}
\langle P\rangle_{W / / G, \boldsymbol{d}} & :=\int_{[\mathbf{Q}(W / / G ; \boldsymbol{d})]^{\mathrm{virt}}} \mathfrak{C W}(P), \\
\langle P\rangle_{W / / G} & :=\sum_{\boldsymbol{d} \in \operatorname{Eff}(W / / G)} e^{\langle\boldsymbol{d}, \boldsymbol{t}\rangle}\langle P\rangle_{W / / G, \boldsymbol{d}} .
\end{aligned}
$$

Conjecture 10.10. Suppose that $W \subset V$ is the zero locus of $G$ semi-invariant polynomials $f_{i}, i=1, \ldots, r$. Provided with conditions in $\$ 10.1$ and 10.7 , for any $P \in \mathbb{C}[\mathfrak{t}]^{\mathscr{W}}$, the generating function (10.9.3) of quasimap invariants coincides with the correlation function (2.2.4) of the A-twisted gauged linear sigma model up to an overall sign;

$$
\langle P\rangle_{\mathrm{GLSM}}= \pm\langle P\rangle_{W / / G} \text {. }
$$

Here the potential of GLSM is given as a G-invariant function $\sum_{i} f_{i} p_{i}$ of $V \times \mathbb{A}^{r}$ where $p_{i}$ denotes $i$-th coordinate of $\mathbb{A}^{r}$ with $R$-charge 2 .

10.11. By taking $\mathcal{P}^{\prime}$ to be the fiber over a fixed point of the natural $\mathbb{G}_{\mathrm{m}}$-action on the domain curve $\mathbb{P}^{1}$, one can define $\mathbb{G}_{\mathrm{m}}$-equivariant quasimap invariants $\langle P\rangle_{W / / G}^{\mathbb{G}_{\mathrm{m}}}$. If $W$ has an action of an algebraic torus $H$ commuting with the action of $G$, then one can define $H \times \mathbb{G}_{\mathrm{m}}$-equivariant quasimap invariants $\langle P\rangle_{W / / G}^{H \times \mathbb{G}_{\mathrm{m}}}$.

\section{Quasimap spaces for Grassmannians}

11.1. The quasimap space $\mathbf{Q}(\operatorname{Gr}(r, n) ; d)$ classifies pairs $(P, u)$ of a principal $\mathrm{GL}_{r}$-bundle $P$ and a $\mathrm{GL}_{r}$-equivariant map $u$. The choice of a principal $\mathrm{GL}_{r}$-bundle $P$ is equivalent to the choice of a vector bundle $S$ of rank $r$, and the choice of a $\mathrm{GL}_{r}$-equivariant map $u$ is equivalent to the choice of a map $\mathcal{S} \rightarrow \mathcal{O}_{\mathbb{P 1}}^{\oplus n}$, which is a sheaf injection since the generic point must go to the semi-stable locus (but not necessarily a morphism of vector bundles). The choice of a sheaf injection $\mathcal{S} \rightarrow \mathcal{O}_{\mathbb{P} 1}^{\oplus n}$ is equivalent to the choice of a surjection $\mathcal{O}_{\mathbb{P} 1}^{\oplus n} \rightarrow \mathcal{Q}$, where $\mathcal{Q}$ is a coherent sheaf whose Hilbert polynomial is $d+(n-r)(t+1)$. This is the same as the Hilbert polynomial of a locally free sheaf of rank $n-r$ and degree $d$, and one has an isomorphism

$$
\mathrm{Q}(\operatorname{Gr}(r, n) ; d) \cong \operatorname{Quot}_{\mathbb{P}^{1}, d}\left(\mathcal{O}_{\mathbb{P}^{1}}^{\oplus n}, n-r\right) .
$$

11.2. It is shown in [BCFK05, Lemma 1.2] that the subspace Q. $(\operatorname{Gr}(r, n) ; d)$ of $\mathbf{Q}(\operatorname{Gr}(r, n) ; d)$ is decomposed into connected components as

$$
\text { Q. }(\operatorname{Gr}(r, n) ; d)=\coprod_{|\boldsymbol{d}|=d} \mathbf{Q} .(\operatorname{Gr}(r, n) ; \boldsymbol{d}),
$$

where $\boldsymbol{d}=\left(d_{1}, \ldots, d_{r}\right)$ runs over elements of $\mathbb{N}^{r}$ satisfying $|\boldsymbol{d}|:=d_{1}+\cdots+d_{r}=d, d_{1} \leq$ $d_{2} \leq \ldots \leq d_{r}$ and each connected component is isomorphic to the partial flag manifold

$$
\text { Q. }(\operatorname{Gr}(r, n) ; \boldsymbol{d}) \cong \operatorname{Fl}\left(m_{1}, \ldots, m_{k}, r, n\right) \text {, }
$$


where $1 \leq m_{1}<m_{2}<\cdots<m_{k}=r$ denote the jumping indices;

$$
0 \leq d_{1}=\cdots=d_{m_{1}}<d_{m_{1}+1}=\cdots=d_{m+2}<\cdots .
$$

Let $x_{1}, \ldots, x_{r}$ be the Chern roots of the dual of the universal subbundle on $\operatorname{Gr}(r, n)$. We also define $|\boldsymbol{x}|:=\sum_{i=1}^{r} x_{i}$ and $|\boldsymbol{d}|:=\sum_{i=1}^{r} d_{i}$ for $\boldsymbol{d}=\left(d_{1}, \ldots, d_{r}\right)$. The $I$-function can be computed by localization as

$$
I_{\mathrm{Gr}(r, n)}(t ; \mathbf{z})=\sum_{\boldsymbol{d} \in \mathbb{N}^{r}}(-1)^{(r-1)|\boldsymbol{d}|} e^{(|\boldsymbol{d}|+|\boldsymbol{x}| / \mathbf{z}) t} I_{\boldsymbol{d}}(\mathbf{z})
$$

where

$$
I_{\boldsymbol{d}}(\mathbf{z})=\frac{\prod_{1 \leq i<j \leq r}\left(x_{i}-x_{j}+\left(d_{i}-d_{j}\right) \mathrm{z}\right)}{\prod_{1 \leq i<j \leq r}\left(x_{i}-x_{j}\right) \prod_{i=1}^{r} \prod_{j=1}^{n} \prod_{l=1}^{d_{i}}\left(x_{i}+l \mathrm{z}\right)} .
$$

As shown in [BCFK05, page 109], the $I$-function and the $J$-function agrees for $\operatorname{Gr}(r, n)$ just as in the case of projective spaces.

11.3. The Hori-Vafa conjecture [HV] proved in [BCFK05] shows that the $I$-functions of $\left(\mathbb{P}^{n-1}\right)^{r}$ and $\operatorname{Gr}(r, n)$ are related by

$$
I_{\operatorname{Gr}(r, n)}(t ; \mathbf{z})=\left.e^{-\sigma_{1}(r-1) \pi \sqrt{-1} / \mathbf{z}} \frac{\mathcal{D} I_{\left(\mathbb{P}^{n-1}\right)^{r}}(\boldsymbol{t} ; \mathbf{z})}{\boldsymbol{\Delta}}\right|_{t_{i}=t+(r-1) \pi \sqrt{-1}}
$$

where

$$
\mathcal{D}:=\prod_{1 \leq i<j \leq r}\left(z \frac{\partial}{\partial t_{i}}-\mathrm{z} \frac{\partial}{\partial t_{j}}\right)
$$

11.4. As shown in BCFK05, the equivariant $I$-function with respect to the natural action of $H=\left(\mathbb{G}_{\mathrm{m}}\right)^{n}$ on $\operatorname{Mat}(r, n)$ is given by

$$
I_{\mathrm{Gr}(r, n)}^{H}(t ; \mathbf{z})=e^{t \sigma_{1} / z} \sum_{\boldsymbol{d} \in \mathbb{N}^{r}}(-1)^{(r-1)|\boldsymbol{d}|} e^{|\boldsymbol{d}| t} \frac{\prod_{1 \leq i<j \leq r}\left(x_{i}-x_{j}+\left(d_{i}-d_{j}\right) \mathbf{z}\right)}{\prod_{1 \leq i<j \leq r}\left(x_{i}-x_{j}\right) \prod_{i=1}^{r} \prod_{j=1}^{n} \prod_{l=1}^{d_{i}}\left(x_{i}-\lambda_{j}+l \mathbf{z}\right)},
$$

and the factorization gives

$$
\sum_{d=0}^{\infty} e^{d \tau}\left\langle e^{(t-\tau) \sigma_{1} / \mathbf{z}}\right\rangle_{\operatorname{Gr}(r, n), d}^{H \times \mathbb{G}_{\mathrm{m}}}=\int_{\operatorname{Gr}(r, n)}^{H} I_{\operatorname{Gr}(r, n)}^{H}(t ; \mathbf{z}) \cup I_{\operatorname{Gr}(r, n)}^{H}(\tau ;-\mathbf{z})
$$

Here $\sigma_{1}=\sum_{i=1}^{r} x_{i}$ is the $H$-equivariant first Chern class of the vector bundle

$$
\mathcal{S}^{\vee}=\left(\operatorname{Mat}(r, n) \times \mathbb{C}^{r}\right) / / G
$$

on $\operatorname{Gr}(r, n)$, where the $G$-action on $\mathbb{C}^{r}$ is the defining representation.

11.5. Let $\mathcal{V}$ be an equivariant vector bundle on $\operatorname{Gr}(r, n)$ associated with a representation $V$ of $\mathrm{GL}_{r}$. If $\mathcal{V}$ is globally generated and $\operatorname{det} \mathcal{V} \cong \omega_{\mathrm{Gr}(r, n)}^{\vee}$, then the zero $Y:=s^{-1}(0)$ of a general section $s \in H^{0}(\mathcal{V})$ is a smooth Calabi-Yau manifold by a generalization of the theorem of Bertini [Muk92, Theorem 1.10]. 
11.6. Let $\left[\operatorname{Mat}(r, n) / \mathrm{GL}_{r}\right]$ be the quotient stack containing $\operatorname{Gr}(r, n)$ as an open substack. The complete intersection $Y \subset \operatorname{Gr}(r, n)$ is an open substack of $\mathcal{Y}:=\left[Z / \mathrm{GL}_{r}\right]$, where $Z \subset \operatorname{Mat}(r, n)$ is the zero of the map $\widetilde{s}: \operatorname{Mat}(r, n) \rightarrow V$ underlying $s$. Indeed, $Y$ has a GIT quotient description $Y=Z / / \mathrm{GL}_{r}$, which allows us to define $\mathbf{Q}(Y ; d)$ and its virtual fundamental cycle as in Section 10, Let $\mathcal{S}_{\mathcal{Y}}^{\vee}$ be the vector bundle on $\mathcal{Y}$ associated with the defining representation of $\mathrm{GL}_{r}$. Any point $p \in \mathbb{P}^{1}$ determines a map $\operatorname{ev}_{p}: \mathbf{Q}(Y ; d) \rightarrow \mathcal{Y}$ sending $f: \mathbb{P}^{1} \rightarrow \mathcal{Y}$ to $f(p) \in \mathcal{Y}$, and the Chern classes

$$
\sigma_{i}:=c_{i}\left(\mathrm{ev}_{p}^{*} \mathcal{S}_{\mathcal{Y}}^{\vee}\right), \quad i=1, \ldots, r
$$

does not depend on the choice of $p \in \mathbb{P}^{1}$. For $P\left(\sigma_{1}, \ldots, \sigma_{r}\right) \in \mathbb{C}\left[\sigma_{1}, \ldots, \sigma_{r}\right]$, we set

$$
\left\langle P\left(\sigma_{1}, \ldots, \sigma_{r}\right)\right\rangle_{Y, d}:=\int_{[\mathbf{Q}(Y ; \boldsymbol{d})]^{\mathrm{virt}}} P\left(\sigma_{1}, \ldots, \sigma_{r}\right)
$$

and

$$
\left\langle P\left(\sigma_{1}, \ldots, \sigma_{r}\right)\right\rangle_{Y}:=\sum_{d=0}^{\infty} e^{d t}\left\langle P\left(\sigma_{1}, \ldots, \sigma_{r}\right)\right\rangle_{Y, d} .
$$

11.7. The equivariant $I$-function of $Y$ is given by

$$
I_{Y}^{H}(t ; \mathbf{z})=\left.\sum_{\boldsymbol{d} \in \mathbb{N}^{r}} e^{(\boldsymbol{d}+\boldsymbol{x} / \mathbf{z}) \cdot \boldsymbol{t}} I_{\boldsymbol{d}}(\boldsymbol{t} ; \mathbf{z})\right|_{t_{i}=t+(r-1) \pi \sqrt{-1}},
$$

where

$$
I_{\boldsymbol{d}}(\boldsymbol{t} ; \mathbf{z}):=\frac{\prod_{\boldsymbol{\delta} \in \Delta(V)} \prod_{l=1}^{\langle\boldsymbol{\delta}, \boldsymbol{d}\rangle}(\langle\boldsymbol{\delta}, \boldsymbol{x}\rangle+l \mathrm{z}) \prod_{1 \leq i<j \leq r}\left(x_{i}-x_{j}+\left(d_{i}-d_{j}\right) \mathrm{z}\right)}{\prod_{1 \leq i<j \leq r}\left(x_{i}-x_{j}\right) \prod_{i=1}^{r} \prod_{j=1}^{n} \prod_{l=1}^{d_{i}}\left(x_{i}-\lambda_{j}+l \mathrm{z}\right)},
$$

where $\Delta(V)$ denotes the set of weights of $V$ and $\langle\boldsymbol{\delta}, \boldsymbol{x}\rangle$ denotes the first Chern class associated to the weight $\boldsymbol{\delta}$ (expressed in terms of the fundamental weights $x_{1}, \ldots, x_{r}$ of the maximal diagonal torus of $G$ ). Localization with respect to the natural $\mathbb{G}_{\mathrm{m}}$-action on $\mathbf{Q}(\operatorname{Gr}(r, n) ; d)$ shows

$$
\left\langle e^{(t-\tau) \sigma_{1} / \mathbf{z}}\right\rangle_{Y}^{H \times \mathbb{G}_{\mathrm{m}}}=\int_{Y}^{H} I^{H}(t ; \mathbf{z}) \cup I^{H}(\tau ;-\mathbf{z})
$$

just as in (8.5.3).

\section{RESIDUE MIRROR SYMMETRY FOR GRASSMANNIANS}

12.1. We define the abelianized quasimap space for $\operatorname{Gr}(r, n)$ by

$$
\begin{aligned}
\mathrm{Q}^{\mathrm{ab}}(\operatorname{Gr}(r, n) ; d) & :=\coprod_{|\boldsymbol{d}|=d} \mathrm{Q}^{\mathrm{ab}}(\operatorname{Gr}(r, n) ; \boldsymbol{d}), \\
\mathrm{Q}^{\mathrm{ab}}(\operatorname{Gr}(r, n) ; \boldsymbol{d}) & :=\mathrm{Q}\left(\mathbb{P}^{n-1} ; d_{1}\right) \times \cdots \times \mathbf{Q}\left(\mathbb{P}^{n-1} ; d_{r}\right),
\end{aligned}
$$

where $\boldsymbol{d}$ runs over $\boldsymbol{d}=\left(d_{1}, \ldots, d_{r}\right) \in \mathbb{N}^{r}$ such that $|\boldsymbol{d}|:=d_{1}+\cdots+d_{r}=d$. An abelianized quasimap

$$
\varphi\left(z_{1}, z_{2}\right)=\left(\left(\varphi_{i 1}\left(z_{1}, z_{2}\right), \ldots, \varphi_{i n}\left(z_{1}, z_{2}\right)\right) \in \mathbf{Q}\left(\mathbb{P}^{n-1} ; d_{i}\right)\right)_{i=1}^{r}
$$


defines a genuine map of degree $d$ if the matrix $\left(\varphi_{i j}\left(z_{1}, z_{2}\right)\right)_{i, j}$ has rank $r$ for any $\left(z_{1}, z_{2}\right) \neq$ 0 . For $P\left(\sigma_{1}, \ldots, \sigma_{r}\right) \in \mathbb{C}\left[\sigma_{1}, \ldots, \sigma_{r}\right]$, we set

$$
\left\langle P\left(\sigma_{1}, \ldots, \sigma_{r}\right)\right\rangle_{\mathrm{Gr}(r, n), \boldsymbol{d}}^{\mathrm{ab}}:=\frac{1}{r !} \int_{\mathbf{Q}^{\mathrm{ab}}(\operatorname{Gr}(r, n) ; \boldsymbol{d})} \prod_{i \neq j}\left(x_{i}-x_{j}\right) P\left(\sigma_{1}\left(x_{1}, \ldots, x_{r}\right), \ldots, \sigma_{r}\left(x_{1}, \ldots, x_{r}\right)\right),
$$

$$
\left\langle P\left(\sigma_{1}, \ldots, \sigma_{r}\right)\right\rangle_{\mathrm{Gr}(r, n), d}^{\mathrm{ab}}:=\sum_{|\boldsymbol{d}|=d}\left\langle P\left(\sigma_{1}, \ldots, \sigma_{r}\right)\right\rangle_{\mathrm{Gr}(r, n), \boldsymbol{d}}^{\mathrm{ab}},
$$

$$
\left\langle P\left(\sigma_{1}, \ldots, \sigma_{r}\right)\right\rangle_{\mathrm{Gr}(r, n)}^{\mathrm{ab}}:=\sum_{d=0}^{\infty}(-1)^{(r-1) d} q^{d}\left\langle P\left(\sigma_{1}, \ldots, \sigma_{r}\right)\right\rangle_{\mathrm{Gr}(r, n), d}^{\mathrm{ab}} .
$$

12.2. If we set $G:=\mathrm{GL}_{r}$ and $V:=\operatorname{Mat}(r, n)$, where $G$ acts naturally on $V$ and $\mathbb{G}_{\mathrm{m}}$ acts trivially on $V$, then we have $Z_{\boldsymbol{d}}^{\mathrm{vec}}(x)=\prod_{i \neq j}\left(x_{i}-x_{j}\right)$ and $Z_{\boldsymbol{d}}^{\mathrm{mat}}(x)=\prod_{i=1}^{r}\left(x_{i}^{-d_{i}-1}\right)^{n}$, so that (2.2.4) gives the same result as (12.1.4);

$$
\left\langle P\left(\sigma_{1}, \ldots, \sigma_{r}\right)\right\rangle_{\mathrm{GLSM}}=\left\langle P\left(\sigma_{1}, \ldots, \sigma_{r}\right)\right\rangle_{\mathrm{Gr}(r, n)}^{\mathrm{ab}} .
$$

12.3. We write the ring homomorphism $\mathbb{C}\left[\sigma_{1}, \ldots, \sigma_{r}\right] \rightarrow \mathrm{QH}(\operatorname{Gr}(r, n))$ sending $\sigma_{i} \in$ $\mathbb{C}\left[\sigma_{1}, \ldots, \sigma_{r}\right]$ to $\sigma_{i} \in H^{*}(\operatorname{Gr}(r, n) ; \mathbb{C}) \cong \mathbb{C}\left[\sigma_{1}, \ldots, \sigma_{r}\right] /\left(h_{n-r+1}, \ldots, h_{n}\right)$ as $P\left(\sigma_{1}, \ldots, \sigma_{r}\right) \mapsto$ $\stackrel{\circ}{P}\left(\sigma_{1}, \ldots, \sigma_{r}\right)$ just as in the case of $\mathbb{P}^{n-1}$.

Theorem 12.4. For any $P\left(\sigma_{1}, \ldots, \sigma_{r}\right) \in \mathbb{C}\left[\sigma_{1}, \ldots, \sigma_{r}\right]$, one has

$$
\left\langle P\left(\sigma_{1}, \ldots, \sigma_{r}\right)\right\rangle_{\operatorname{Gr}(r, n)}^{\mathrm{ab}}=\int_{\operatorname{Gr}(r, n)} \stackrel{\circ}{P}\left(\sigma_{1}, \ldots, \sigma_{r}\right) .
$$

Proof. It follows from (3.7.2) that

$$
\begin{aligned}
\langle P & \left.\left(\sigma_{1}, \ldots, \sigma_{r}\right)\right\rangle_{\mathrm{Gr}(r, n)}^{\mathrm{ab}} \\
& =\frac{1}{r !} \sum_{d_{1}, \ldots, d_{r}=0}^{\infty}\left((-1)^{r-1} q\right)^{d_{1}+\cdots+d_{r}} \operatorname{Res} \prod_{i \neq j}\left(x_{i}-x_{j}\right) P\left(\sigma_{1}, \ldots, \sigma_{r}\right) \frac{d x_{1}}{x_{1}^{n\left(d_{1}+1\right)}} \wedge \cdots \wedge \frac{d x_{r}}{x_{r}^{n\left(d_{r}+1\right)}} \\
& =\frac{1}{r !} \operatorname{Res} \prod_{i \neq j}\left(x_{i}-x_{j}\right) P\left(\sigma_{1}, \ldots, \sigma_{r}\right) \frac{d x_{1}}{x_{1}^{n}+(-1)^{r} q} \wedge \cdots \wedge \frac{d x_{r}}{x_{r}^{n}+(-1)^{r} q} \\
& =\frac{1}{r ! n^{r}} \sum_{x_{1}^{n}=(-1)^{r-1} q} \ldots \sum_{x_{r}^{n}=(-1)^{r-1} q} \prod_{i \neq j}\left(x_{i}-x_{j}\right) P\left(\sigma_{1}\left(x_{1}, \ldots, x_{r}\right), \ldots, \sigma_{r}\left(x_{1}, \ldots, x_{r}\right)\right) \\
& =\int_{\operatorname{Gr}(r, n)} \stackrel{\circ}{P}\left(\sigma_{1}, \ldots, \sigma_{r}\right),
\end{aligned}
$$

where the last equality is the Vafa-Intriligator formula [ST97, Theorem 4.6].

12.5. Theorem 12.4 is related to intersection theory on the moduli space of vector bundles on a Riemann surface through a theorem of Witten [Wit95, which states the existence of a ring isomorphism $\mathrm{QH}(\operatorname{Gr}(r, n)) /(q-1) \stackrel{\sim}{\rightarrow} R(U(r))_{n-r, n}$ from the quantum cohomology of $\operatorname{Gr}(r, n)$ at $q=1$ and the Verlinde algebra of $U(r)$ at $\mathrm{SU}(r)$ level $n-r$ and $U(1)$ level $n$. 
12.6. We define the $\mathbb{G}_{\mathrm{m}}$-equivariant correlator of $P\left(\sigma_{1}, \ldots, \sigma_{r}\right) \in \mathbb{C}\left[\sigma_{1}, \ldots, \sigma_{r}\right]$ by

$$
\left\langle P\left(\sigma_{1}, \ldots, \sigma_{r}\right)\right\rangle_{\operatorname{Gr}(r, n)}^{\mathrm{ab}, \mathbb{G}_{\mathrm{m}}}:=\left.\sum_{\boldsymbol{d} \in \mathbb{N}^{r}} e^{\boldsymbol{d} \cdot \boldsymbol{t}}\left\langle P\left(\sigma_{1}, \ldots, \sigma_{r}\right)\right\rangle_{\mathrm{Gr}(r, n), \boldsymbol{d}}^{\mathrm{ab}, \mathbb{G}_{\mathrm{m}}}\right|_{t_{i}=t+(r-1) \pi \sqrt{-1}}
$$

where

$$
\begin{array}{r}
\left\langle P\left(\sigma_{1}, \ldots, \sigma_{r}\right)\right\rangle_{\mathrm{Gr}(r, n), \boldsymbol{d}}^{\mathrm{ab}, \mathbb{G}_{\mathrm{m}}}:=\int_{\mathrm{Q}^{\mathrm{ab}}(\operatorname{Gr}(r, n) ; \boldsymbol{d})}^{\mathbb{G}_{\mathrm{m}}} \prod_{1 \leq i<j \leq n}\left(x_{i}-x_{j}\right)\left(x_{j}-x_{i}+\left(d_{j}-d_{i}\right) \mathrm{z}\right) \\
P\left(\sigma_{1}\left(x_{1}, \ldots, x_{r}\right), \ldots, \sigma_{r}\left(x_{1}, \ldots, x_{r}\right)\right) .
\end{array}
$$

Since $\mathbf{Q}\left(\left(\mathbb{P}^{n-1}\right)^{r} ; \boldsymbol{d}\right)^{\mathbb{G}_{m}}=\prod_{i=1}^{r} \mathbf{Q}\left(\mathbb{P}^{n-1} ; d_{i}\right)^{\mathbb{G}_{m}}$ under $\mathbf{Q}\left(\left(\mathbb{P}^{n-1}\right)^{r} ; \boldsymbol{d}\right)=\prod_{i=1}^{r} \mathbf{Q}\left(\mathbb{P}^{n-1} ; d_{i}\right)$, we have a straightforward generalization of (3.12.2):

$$
\sum_{\boldsymbol{d} \in \mathbb{N}^{r}} e^{\boldsymbol{d} \cdot \boldsymbol{\tau}}\left\langle e^{(\boldsymbol{t}-\boldsymbol{\tau}) \cdot \boldsymbol{x} / z}\right\rangle_{\left(\mathbb{P}^{n-1}\right)^{r}, \boldsymbol{d}}^{\mathbb{G}_{\mathrm{m}}}=\int_{\left(\mathbb{P}^{n-1}\right)^{r}} I_{\left(\mathbb{P}^{n-1}\right)^{r}}(\boldsymbol{t} ; \mathbf{z}) \cup I_{\left(\mathbb{P}^{n-1}\right)^{r}}(\boldsymbol{\tau} ;-\mathbf{z}),
$$

By acting $\mathcal{D}_{t}:=\prod_{1 \leq i<j \leq r}\left(z \partial_{t_{i}}-\mathrm{z} \partial_{t_{j}}\right)$ and $-\mathcal{D}_{\tau}:=\prod_{1 \leq i<j \leq r}\left(-\mathrm{z} \partial_{\tau_{i}}+\mathrm{z} \partial_{\tau_{j}}\right)$ on both sides of (12.6.3) one obtains

$$
\begin{aligned}
\sum_{\boldsymbol{d} \in \mathbb{N}^{r}} e^{\boldsymbol{d} \cdot \boldsymbol{t}} & \left\langle\prod_{1 \leq i<j \leq r}\left(x_{i}-x_{j}\right) \prod_{1 \leq i<j \leq r}\left(\left(x_{j}+d_{j} \mathbf{z}\right)-\left(x_{i}+d_{i} \mathbf{z}\right)\right) \cdot e^{(\boldsymbol{t}-\boldsymbol{\tau}) \cdot \boldsymbol{x} / z}\right\rangle_{\left(\mathbb{P}^{n-1}\right)^{r}}^{\mathbb{G}_{\mathrm{m}}} \\
& =\left\langle e^{(\boldsymbol{t}-\boldsymbol{\tau}) \cdot \boldsymbol{x} / z}\right\rangle_{\operatorname{Gr}(r, n)}^{\mathrm{ab}, \mathbb{G}_{m}}
\end{aligned}
$$

on the left hand side and

$$
\int_{\left(\mathbb{P}^{n-1}\right)^{r}} \mathcal{D}_{\boldsymbol{t}} I_{\left(\mathbb{P}^{n-1}\right)^{r}}(\boldsymbol{t} ; \mathbf{z}) \cup\left(-\mathcal{D}_{\boldsymbol{\tau}}\right) I_{\left(\mathbb{P}^{n-1}\right)^{r}}(\boldsymbol{\tau} ;-\mathbf{z})
$$

on the right hand side. By setting $t_{i}=t+(r-1) \pi \sqrt{-1}, \tau_{i}=\tau+(r-1) \pi \sqrt{-1}$ and using (11.3.1), one obtains

$$
\begin{aligned}
\left\langle e^{(t-\tau) \sigma_{1} / \mathbf{z}}\right\rangle_{\operatorname{Gr}(r, n)}^{\mathrm{ab}, \mathbb{G}_{\mathrm{m}}} & =\frac{1}{r !} \int_{\left(\mathbb{P}^{n-1}\right)^{r}} \Delta \cup I_{\mathrm{Gr}(r, n)}(t ; \mathbf{z}) \cup \Delta \cup I_{\operatorname{Gr}(r, n)}(\tau ;-\mathbf{z}) \\
& =\int_{\operatorname{Gr}(r, n)} I_{\operatorname{Gr}(r, n)}(t ; \mathbf{z}) \cup I_{\operatorname{Gr}(r, n)}(\tau ;-\mathbf{z}),
\end{aligned}
$$

where the last equality is Martin's formula (9.5.2). On the other hand, localization with respect to the natural $\mathbb{G}_{\mathrm{m}}$-action on the domain curve gives the factorization

$$
\left\langle e^{(t-\tau) \sigma_{1} / \mathbf{z}}\right\rangle_{\operatorname{Gr}(r, n)}^{\mathbb{G}_{\mathrm{m}}}=\int_{\operatorname{Gr}(r, n)} I_{\operatorname{Gr}(r, n)}(t ; \mathbf{z}) \cup I_{\operatorname{Gr}(r, n)}(\tau ;-\mathbf{z}) .
$$

Together with (12.6.7), this gives the equality

$$
\left\langle e^{(t-\tau) \sigma_{1} / z}\right\rangle_{\operatorname{Gr}(r, n)}^{\mathrm{ab}, \mathbb{G}_{\mathrm{m}}}=\left\langle e^{(t-\tau) \sigma_{1} / \mathrm{z}}\right\rangle_{\mathrm{Gr}(r, n)}^{\mathbb{G}_{\mathrm{m}}}
$$

of the abelianized correlator and the ordinary correlator. 
For any $P(\boldsymbol{x}) \in \mathbb{C}\left[x_{1}, \ldots, x_{r}\right]^{\mathfrak{S}_{r}}$, the same argument gives

$$
\begin{aligned}
\langle P & \left.(\boldsymbol{x}) e^{(\boldsymbol{t}-\boldsymbol{\tau}) \cdot \boldsymbol{x} / z}\right\rangle_{\mathrm{Gr}(r, n)}^{\mathrm{ab}, \mathbb{G}_{\mathrm{m}}} \\
& =\int_{\mathrm{Gr}(r, n)}\left(\sum_{\boldsymbol{d} \in \mathbb{N}^{r}} P(\boldsymbol{x}+\boldsymbol{d} \mathbf{z}) I_{\mathrm{Gr}(r, n), \boldsymbol{d}}(\boldsymbol{t} ; \mathbf{z})\right) \cup\left(\sum_{\boldsymbol{d} \in \mathbb{N}^{r}} I_{\mathrm{Gr}(r, n), \boldsymbol{d}}(\boldsymbol{\tau} ;-\mathbf{z})\right) \\
& =\left\langle P(\boldsymbol{x}) e^{(\boldsymbol{t}-\boldsymbol{\tau}) \cdot \boldsymbol{x} / z}\right\rangle_{\mathrm{Gr}(r, n)}^{\mathbb{G}_{\mathrm{m}}}
\end{aligned}
$$

where $t_{i}=t+(r-1) \pi \sqrt{-1}$ and $\tau_{i}=\tau+(r-1) \pi \sqrt{-1}$. By setting $t=\tau$ in (12.6.10), one obtains

$$
\langle P(\boldsymbol{x})\rangle_{\mathrm{Gr}(r, n)}^{\mathrm{ab}, \mathbb{G}_{\mathrm{m}}}=\langle P(\boldsymbol{x})\rangle_{\mathrm{Gr}(r, n)}^{\mathbb{G}_{\mathrm{m}}} .
$$

Together with (12.2.1), this proves Conjecture 10.10 for Grassmannians.

12.7. Let $Y \subset \operatorname{Gr}(r, n)$ be the zero locus of a general section of a globally-generated vector bundle $\mathcal{V}$ on $\operatorname{Gr}(r, n)$ associated with a representation $V$ of $\mathrm{GL}_{r}$. We define the abelianized $\mathbb{G}_{\mathrm{m}}$-equivariant Morrison-Plesser class of $Y$ by

$$
\Phi_{\boldsymbol{d}}^{\mathrm{ab}, \mathbb{G}_{\mathrm{m}}}(Y ; \mathrm{z}):=\prod_{\boldsymbol{\delta} \in \Delta(V)} \prod_{l=1}^{\langle\boldsymbol{\delta}, \boldsymbol{d}\rangle}(\langle\boldsymbol{\delta}, \boldsymbol{x}\rangle+l \mathrm{z}) .
$$

For $P \in \mathbb{C}\left[\sigma_{1}, \ldots, \sigma_{r}\right]$, we set

$$
\left\langle P\left(\sigma_{1}, \ldots, \sigma_{r}\right)\right\rangle_{Y}^{\mathrm{ab}, \mathbb{G}_{\mathrm{m}}}:=\sum_{\boldsymbol{d} \in \mathbb{N}^{r}} q^{|\boldsymbol{d}|}\left\langle P\left(\sigma_{1}, \ldots, \sigma_{r}\right) \Phi_{\boldsymbol{d}}^{\mathrm{ab}, \mathbb{G}_{\mathrm{m}}}(Y ; \mathbf{z}) v\right\rangle_{\operatorname{Gr}(r, n), \boldsymbol{d}}^{\mathrm{ab}, \mathbb{G}_{\mathrm{m}}},
$$

where $v:=\prod_{\delta \in \Delta(V)}\langle\boldsymbol{\delta}, \boldsymbol{x}\rangle$ is the Euler class of the normal bundle of $Y$ in $\operatorname{Gr}(r, n)$. By the same reasoning as in Section 12.6 with the insertion of the abelizanized Morrison-Plesser class, one obtains

$$
\left\langle P\left(\sigma_{1}, \ldots, \sigma_{r}\right)\right\rangle_{Y}=(-1)^{|\Delta(V)|}\left\langle P\left(\sigma_{1}, \ldots, \sigma_{r}\right)\right\rangle_{\mathrm{GLSM}} .
$$

Here, the identification between $q$ and the Fayet-Illiopoulos parameter $t^{\prime}$ is given by

$$
q=(-1)^{\sum_{\delta \in \Delta(V)}\langle\boldsymbol{\delta}, \mathbf{1}\rangle} e^{t^{\prime}}
$$

where $\mathbf{1}:=(1, \cdots, 1) \in \mathbb{N}^{r}$.

12.8. As an example, consider the vector bundle of rank 3 on $\operatorname{Gr}(3,5)=\operatorname{Mat}(3,5) / / U(3)$ associated with the representation of $U(3)$ determined by the Young diagram

$$
\lambda=\square \text {. }
$$

This vector bundle is the tensor product $\wedge^{2} \mathcal{Q}(1)$ of the second exterior power $\wedge^{2} \mathcal{Q}$ of the universal quotient bundle $\mathcal{Q}$ on $\operatorname{Gr}(2,5) \cong \operatorname{Gr}(3,5)$ and the ample generator $\mathcal{O}(1)$ of the Picard group. One can immediately see from the Young diagram that the restriction of the representation of $U(3)$ associated with $\lambda$ to the diagonal maximal torus $T \cong\left(\mathbb{G}_{\mathrm{m}}\right)^{3}$ is the direct sum $\rho_{1,2,2} \oplus \rho_{2,1,2} \oplus \rho_{2,2,1}$. The associated line bundle on the abelian quotient $\left(\mathbb{P}^{4}\right)^{3}$ is given by $\mathcal{O}(1,2,2) \oplus \mathcal{O}(2,1,2) \oplus \mathcal{O}(2,2,1)$.

The complete intersection in $\operatorname{Gr}(3,5)$ defined by $\wedge^{2} \mathcal{Q}(1)$ is a Calabi-Yau 3-fold of Picard number 1, which will be denoted by $Y$ henceforth. The Euler class of the normal bundle of $Y$ is

$$
v:=\left(x_{1}+2 x_{2}+2 x_{3}\right)\left(2 x_{1}+x_{2}+2 x_{3}\right)\left(2 x_{1}+2 x_{2}+x_{3}\right),
$$


the abelianized Morrison-Plesser class is

$$
\begin{aligned}
\Phi^{\mathrm{ab}}(Y ; \boldsymbol{d}):=\left(x_{1}+2 x_{2}+2 x_{3}\right)^{d_{1}+2 d_{2}+2 d_{3}} \\
\left(2 x_{1}+x_{2}+2 x_{3}\right)^{2 d_{1}+d_{2}+2 d_{3}}\left(2 x_{1}+2 x_{2}+x_{3}\right)^{2 d_{1}+2 d_{2}+d_{3}},
\end{aligned}
$$

and the generating function for $\sigma_{1}^{3}$ is

$$
\begin{aligned}
\left\langle\sigma_{1}^{3}\right\rangle_{Y}^{\mathrm{ab}}=-\frac{1}{6} \sum_{d_{1}=0}^{\infty} & \sum_{d_{2}=0}^{\infty} \sum_{d_{3}=0}^{\infty} q^{d_{1}+d_{2}+d_{3}} \operatorname{Res}\left(x_{1}+x_{2}+x_{3}\right)^{3} \\
& \left(x_{1}-x_{2}\right)^{2}\left(x_{1}-x_{3}\right)^{2}\left(x_{2}-x_{3}\right)^{2} \Phi^{\mathrm{ab}}(Y ; \boldsymbol{d}) v \frac{d x_{1}}{x_{1}^{n\left(d_{1}+1\right)}} \wedge \frac{d x_{2}}{x_{2}^{n\left(d_{2}+1\right)}} \wedge \frac{d x_{3}}{x_{3}^{n\left(d_{3}+1\right)}}
\end{aligned}
$$

$$
=\frac{25(1-q)}{(1+q)\left(1-123 q+q^{2}\right)} .
$$

This matches the Yukawa coupling of the mirror computed by Miura [Miu13, §5.2].

12.9. When $\mathcal{V}$ is a direct sum of line bundles, the mirror of $Y$ is constructed by toric degenerations BCFKvS98, BCFKvS00. It is an interesting problem to compare the generating function (11.6.3) with the Yukawa coupling of this mirror.

\section{Bethe/Gauge Correspondence}

13.1. Let $V_{1}$ and $W_{1}$ be Hermitian vector spaces of dimensions $r$ and $n$. The unitary group $U(r)$ acts naturally on $V_{1}$ and trivially on $W_{1}$, inducing an action on $T^{*} \operatorname{Hom}\left(V_{1}, W_{1}\right) \cong$ $\operatorname{Hom}\left(V_{1}, W_{1}\right) \oplus \operatorname{Hom}\left(W_{1}, V_{1}\right)$. The real and complex moment maps for this action are given by

$$
\begin{array}{ll}
\mu_{\mathbb{R}}: \operatorname{Hom}\left(W_{1}, V_{1}\right) \oplus \operatorname{Hom}\left(V_{1}, W_{1}\right) \rightarrow \operatorname{End}\left(V_{1}\right), \quad\left(i_{1}, j_{1}\right) \mapsto \frac{\sqrt{-1}}{2}\left(i_{1} i_{1}^{*}-j_{1}^{*} j_{1}\right), \\
\mu_{\mathbb{C}}: \operatorname{Hom}\left(W_{1}, V_{1}\right) \oplus \operatorname{Hom}\left(V_{1}, W_{1}\right) \rightarrow \operatorname{End}\left(V_{1}\right), \quad\left(i_{1}, j_{1}\right) \mapsto i_{1} j_{1} .
\end{array}
$$

If $\left(i_{1}, j_{1}\right) \in \mu_{\mathbb{R}}^{-1}\left(\zeta \sqrt{-1} \mathrm{id}_{V_{1}}\right)$ for $\zeta<0$, then $j_{1}$ is injective. If $\left(i_{1}, j_{1}\right) \in \mu_{\mathbb{C}}^{-1}(0)$, then $i_{1}$ descends to a map $W_{1} / \operatorname{Im} j_{1} \rightarrow V_{1}$. It follows that the hyperKähler quotient is isomorphic to $T^{*} \operatorname{Gr}(r, n)$;

$$
\left(\zeta_{\mathbb{R}}^{-1}\left(\zeta \sqrt{-1} \operatorname{id}_{V_{1}}\right) \cap \mu_{\mathbb{C}}^{-1}(0)\right) / U(r) \cong T^{*} \operatorname{Gr}(r, n) .
$$

This suggests that the gauged linear sigma model with the gauge group $U(r)$ and the reprensetation $V:=\operatorname{Hom}\left(W_{1}, V_{1}\right) \oplus \operatorname{Hom}\left(V_{1}, W_{1}\right) \oplus \operatorname{End}\left(V_{1}\right)$ describes the quantum cohomology of $T^{*} \operatorname{Gr}(r, n)$. Here $\operatorname{End}\left(V_{1}\right)$ is the Lagrange multiplier for the complex moment map equation, and the potential is given by

$$
V \ni\left(i_{1}, j_{1}, P\right) \mapsto \operatorname{tr}\left(P i_{1} j_{1}\right)
$$

Let $H:=H_{1} \times H_{2}$ be the product of

- the diagonal maximal torus $H_{1}$ of $U(n)$, acting on $\operatorname{Hom}\left(W_{1}, V_{1}\right)$ and $\operatorname{Hom}\left(V_{1}, W_{1}\right)$ through the natural action on $W_{1}$, and trivially on $\operatorname{End}\left(V_{1}\right)$, and

- the group $H_{2}=U(1)$ acting trivially on $\operatorname{Hom}\left(W_{1}, V_{1}\right)$, by scalar multiplication on $\operatorname{Hom}\left(V_{1}, W_{1}\right)$, and by inverse scalar multiplication on $\operatorname{End}\left(V_{1}\right)$. 
One has

$$
\begin{aligned}
Z_{d}^{\mathrm{vec}}(x)= & \prod_{1 \leq i \neq j \leq r}\left(x_{i}-x_{j}\right), \\
Z_{d}^{\mathrm{mat}}(x)= & \prod_{j=1}^{n} \prod_{i=1}^{r}\left(x_{i}-\lambda_{j}\right)^{-d_{i}-1} \\
& \times \prod_{j=1}^{n} \prod_{i=1}^{r}\left(-x_{i}+\lambda_{j}-\mu\right)^{-\left(-d_{i}\right)-1} \\
& \times \prod_{1 \leq i \neq j \leq r}\left(x_{i}-x_{j}+\mu\right)^{2-\left(d_{i}-d_{j}\right)-1},
\end{aligned}
$$

so that the $H$-equivariant correlator of $P \in \mathbb{C}\left[x_{1}, \ldots, x_{r}\right]^{\mathfrak{S}_{r}}$ is given by

$$
\begin{aligned}
\langle P\rangle_{\mathrm{GLSM}}^{H}=\frac{1}{r !} \sum_{d_{1}=0}^{\infty} & \cdots \sum_{d_{r}=0}^{\infty}\left((-1)^{r-1} e^{t}\right)^{d_{1}+\cdots+d_{r}} \\
\operatorname{Res}\left[\frac{\prod_{1 \leq i \neq j \leq r}\left(x_{i}-x_{j}\right)}{\prod_{1 \leq i, j \leq r}\left(x_{i}-x_{j}+\mu\right)^{\left(d_{i}-d_{j}-1\right)}}\right. & \left.\frac{\prod_{j=1}^{n} \prod_{i=1}^{r}\left(-x_{i}+\lambda_{j}-\mu\right)^{d_{i}-1}}{\prod_{j=1}^{n} \prod_{i=1}^{r}\left(x_{i}-\lambda_{j}\right)^{d_{i}+1}} P d x_{1} \wedge \cdots \wedge d x_{r}\right],
\end{aligned}
$$

where Res denotes the sum of residues at the points where $x_{i}$ is one of $\lambda_{j}$ for $i=1, \ldots, r$ and $j=1, \ldots, n$ (there are $n^{r}$ such points). This can formally be regarded as an equivariant integration over the projective space of dimension $\sum_{i=1}^{r}\left(d_{i}+1\right)-1$, and it is an interesting problem to give a geometric interpretation.

The effective potential (2.4.1) of this gauged linear sigma model is given by

$$
\begin{aligned}
& W_{\mathrm{eff}}(\boldsymbol{x} ; t)=W_{\mathrm{FI}}\left(\boldsymbol{x} ; t^{\prime}\right)+W_{\mathrm{vec}}(\boldsymbol{x})+W_{\mathrm{mat}}(\boldsymbol{x}), \\
& W_{\mathrm{FI}}(\boldsymbol{x} ; t)=t\left(x_{1}+\cdots+x_{r}\right) \\
& W_{\mathrm{vec}}(\boldsymbol{x})=-\pi \sqrt{-1} \sum_{1 \leq i<j \leq r}\left(x_{j}-x_{i}\right) \\
&=-\pi \sqrt{-1} \sum_{i=1}^{r}(2 i-r-1) x_{i}, \\
& W_{\mathrm{mat}}(\boldsymbol{x})=-\sum_{i=1}^{r} \sum_{j=1}^{n}\left(x_{i}-\lambda_{j}\right)\left(\log \left(x_{i}-\lambda_{j}\right)-1\right) \\
& \quad-\sum_{i=1}^{r} \sum_{j=1}^{n}\left(-x_{i}+\lambda_{j}-\mu\right)\left(\log \left(-x_{i}+\lambda_{j}-\mu\right)-1\right) \\
& \quad-\sum_{i=1}^{r} \sum_{j=1}^{r}\left(x_{i}-x_{j}+\mu\right)\left(\log \left(x_{i}-x_{j}+\mu\right)-1\right),
\end{aligned}
$$


where $\lambda_{j}$ and $\mu$ are equivariant parameters for the actions of $H_{1}$ and $H_{2}$ respectively. Note that

$$
\begin{aligned}
e^{\partial W_{\mathrm{eff}} / \partial x_{i}} & =e^{t} \cdot(-1)^{2 i-r-1} \cdot \prod_{j=1}^{n}\left(x_{i}-\lambda_{j}\right)^{-1} \prod_{j=1}^{n}\left(-x_{i}+\lambda_{j}-\mu\right) \prod_{j \neq i} \frac{x_{j}-x_{i}+\mu}{x_{i}-x_{j}+\mu} \\
& =e^{t+n \pi \sqrt{-1}} \prod_{j=1}^{n} \frac{x_{i}-\lambda_{j}+\mu}{x_{i}-\lambda_{j}} \prod_{j \neq i} \frac{x_{i}-x_{j}-\mu}{x_{i}-x_{j}+\mu},
\end{aligned}
$$

so that the equations $e^{\partial_{x_{i}} W_{\mathrm{eff}}}=1, i=1, \ldots, r$ gives

$$
\prod_{j=1}^{n} \frac{x_{i}-\lambda_{j}}{x_{i}-\lambda_{j}+\mu}=e^{t+n \pi \sqrt{-1}} \prod_{j \neq i} \frac{x_{i}-x_{j}-\mu}{x_{i}-x_{j}+\mu} .
$$

By taking the sum over $d_{i}$ just as in the proof of Corollary 3.7, one obtains

$$
\begin{aligned}
\langle P\rangle_{\mathrm{GLSM}}^{H}=\frac{1}{r !} \operatorname{Res} & {\left[\frac{1}{\prod_{i=1}^{r}\left(\left(1-e^{\partial_{x_{i}} W_{\mathrm{eff}}}\right) \prod_{j=1}^{n}\left(x_{i}-\lambda_{j}\right)\right)}\right.} \\
& \left.\frac{\prod_{1 \leq i \neq j \leq r}\left(x_{i}-x_{j}\right) \prod_{1 \leq i, j \leq r}\left(x_{i}-x_{j}+\mu\right)}{\prod_{i=1}^{r} \prod_{j=1}^{n}\left(-x_{i}+\lambda_{j}-\mu\right)} P d x_{1} \wedge \cdots \wedge d x_{r}\right]
\end{aligned}
$$

where Res denotes the sum of residues at the roots of the equations (13.1.16).

13.2. The Heisenberg model, also known as the homogeneous $X X X_{\frac{1}{2}}$ model, is the $\mathrm{SU}(2)$ spin chain model with Hamiltonian

$$
H=\sum_{i=1}^{n} \boldsymbol{S}_{i} \cdot \boldsymbol{S}_{i+1}
$$

where $\boldsymbol{S}_{i}=\left(S_{i}^{x}, S_{i}^{y}, S_{i}^{z}\right)=\left(\sigma_{i}^{x} / 2, \sigma_{i}^{y} / 2, \sigma_{i}^{z} / 2\right)$ are halves of Pauli matrices acting on the $i$-th factor of the Hilbert space $\mathcal{H}:=\left(\mathbb{C}^{2}\right)^{\otimes n}$ and

$$
\boldsymbol{S}_{i} \cdot \boldsymbol{S}_{i+1}:=S_{i}^{x} S_{i+1}^{x}+S_{i}^{y} S_{i+1}^{y}+S_{i}^{z} S_{i+1}^{z} .
$$

The total spin

$$
S^{z}:=\sum_{i=1}^{n} S_{i}^{z}
$$

clearly commutes with the Hamiltonian, and we restrict to the $S^{z}$-eigenspace $\mathcal{H}_{r} \subset \mathcal{H}$ with eigenvalue $(-n+r) / 2$. We impose the quasi-periodicity condition

$$
\boldsymbol{S}_{n+1}=e^{\sqrt{-1} \vartheta S_{1}^{z}} \boldsymbol{S}_{1} e^{-\sqrt{-1} \vartheta S_{1}^{z}} .
$$

Introduce variables $\boldsymbol{x}=\left(x_{1}, \ldots, x_{r}\right)$ related to quasi-momenta $\boldsymbol{p}=\left(p_{1}, \ldots, p_{r}\right)$ by

$$
e^{\sqrt{-1} p_{i}}=\frac{x_{i}+\frac{\sqrt{-1}}{2}}{x_{i}-\frac{\sqrt{-1}}{2}} .
$$

Then $H$-eigenspaces in $\mathcal{H}_{r}$ correspond bijectively to solutions of the Bethe equation

$$
\left(\frac{x_{i}+\frac{\sqrt{-1}}{2}}{x_{i}-\frac{\sqrt{-1}}{2}}\right)^{n}=e^{\sqrt{-1} \vartheta} \prod_{j \neq i} \frac{x_{i}-x_{j}+\sqrt{-1}}{x_{i}-x_{j}-\sqrt{-1}}
$$




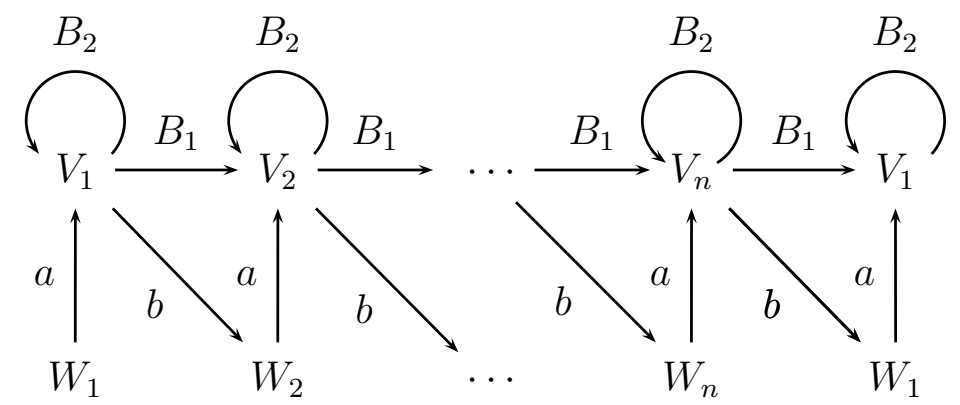

FiguRE 14.1. The chainsaw quiver

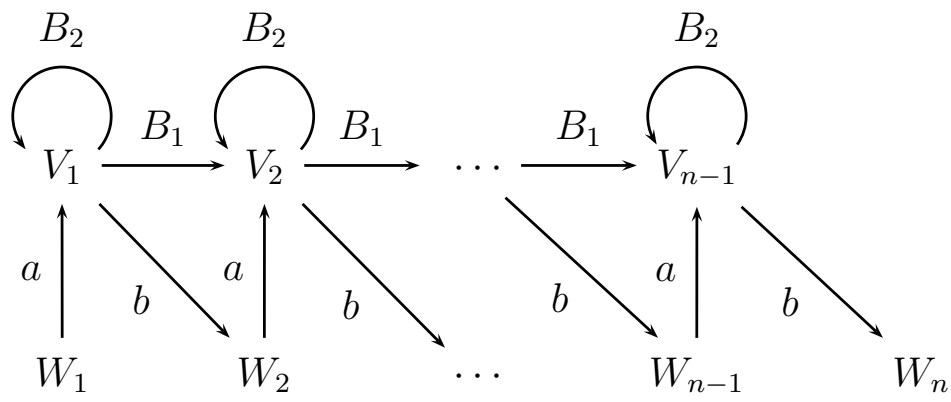

FiguRE 14.2. The handsaw quiver

with eigenavlues $n-2 r+2 \sum_{i=1}^{r} \cos p_{i}$. The integrability comes from factorization of many-body S-matrix into the product of the 2-body S-matrix given by

$$
S\left(p_{i}, p_{j}\right)=1-2 e^{\sqrt{-1} p_{j}}+e^{\sqrt{-1}\left(p_{i}+p_{j}\right)} .
$$

See e.g. Sta12 and references therein for Bethe ansatz for the quasi-periodic Heisenberg model. The Bethe equation (13.2.6) coincides with (13.1.16) under $\lambda_{j}=\frac{\sqrt{-1}}{2}$, $j=1, \ldots, n, \mu=-\sqrt{-1}$, and $\vartheta=-\sqrt{-1} t+n / 2$. This observation and its generalizations is called Bethe/gauge correspondence [NS09]. The relation between classical/quantum cohomology of Grassmannians and integrable systems is studied in BMO11, MO19, GRTV13, Oko17.

\section{QUASIMAPS AND INSTANTONS}

14.1. As explained in [FR14, Section 2.3], the moduli space of framed instantons on $\mathbb{C} \times[\mathbb{C} /(\mathbb{Z} / n \mathbb{Z})]$ is isomorphic to the Nakajima quiver variety associated with the chainsaw quiver shown in Figure 14.1.

14.2. Representations of the chainsaw quiver satisfying $\operatorname{dim} V_{n}=0$ are in one-to-one correspondence with representations of the handsaw quiver shown in Figure 14.2. It is shown in [FR14, Section 2.3] (see also [Nak12, Section 3] for an exposition) that the Nakajima quiver variety associated with the handsaw quiver is isomorphic to the parabolic Laumon space parametrizing flags

$$
0=E_{0} \subset E_{1} \subset \cdots \subset E_{n-1} \subset E_{n}=W \otimes_{\mathbb{C}} \mathcal{O}_{\mathbb{P}^{1}}
$$

of locally free sheaves on $\mathbb{P}^{1}$ such that $\operatorname{rank} E_{i}=\sum_{j \leq i} \operatorname{dim} W_{j}, \operatorname{deg} E_{i}=-\operatorname{dim} V_{i}$, and the flag at $\infty \in \mathbb{P}^{1}$ is equal to the standard flag $0 \subset W_{1} \subset W_{1} \oplus W_{2} \subset \cdots \subset W_{1} \oplus$ 
$W_{2} \oplus \cdots \oplus W_{n-1} \subset W$. This coincides with the space of based quasimaps to partial flag varieties, i.e., quasimaps with specified value at infinity.

\section{QuASIMAPS AND MONOPOLES}

15.1. Let $G$ be a compact Lie group with a maximal torus $H$. A monopole on $\mathbb{R}^{3}$ is a pair $(A, \Phi)$ of a connection $A$ on a principal $G$-bundle $P$ and a section $\Phi$ of $P \times_{G} \mathfrak{g}$ satisfying the Bogomolny equation

$$
F_{A}=* d_{A} \Phi
$$

In order for the curvature to have a finite $L^{2}$-norm, it is natural to demand that the restriction of $\Phi$ to a sphere with large radius tends to a map to a fixed adjoint orbit $\mathcal{O} \cong G / H \cong G_{\mathbb{C}} / P$. The homotopy class $\boldsymbol{k} \in \pi_{2}\left(G_{\mathbb{C}} / P\right)$ of the resulting map is called the charge of the monopole.

15.2. A choice of a gauge satisfying a certain boundary condition at infinity is called a framing of the monopole. The framed moduli space is a principal $H$-bundle over the unframed moduli space. The framed moduli space has a natural hyperKähler structure coming from the dimensional reduction of the anti-self-dual equation in dimension 4 .

15.3. Monopoles on $\mathbb{R}^{3}$ are related to

(1) spectral curves on $T \mathbb{P}^{1}$,

(2) Nahm's equation

$$
\frac{d T_{i}}{d s}=\epsilon_{i j k}\left[T_{j}, T_{k}\right], \quad i=1,2,3
$$

for $T_{i} \in C^{\infty}((0,2), \operatorname{Mat}(k, k ; \mathbb{C}))$, and

(3) based quasimaps from $\mathbb{P}^{1}$ to $G_{\mathbb{C}} / P$ of degree $\boldsymbol{k}$.

(11) comes from the twistor correspondence [Hit82, Hit83], and (2) comes from Nahm transform [Nah82. (3) is proved for SU(2) in [Don84, and the general case can be found in [Jar98b, Jar98a] and references therein.

\section{Quasimaps AND VORTICES}

16.1. Let $X$ be a Kähler manifold, $(E, h)$ be a Hermitian vector bundle on $X$, and $\tau$ be a positive real number. The Yang-Mills-Higgs functional sends a pair $(A, \phi)$ of a unitary connection $d_{A}$ of $(E, h)$ and a section $\phi$ of $E$ to

$$
\mathscr{Y} \mathscr{H} \mathscr{H}(A, \phi)=\left\|F_{A}\right\|_{L^{2}}^{2}+\left\|d_{A} \phi\right\|_{L^{2}}^{2}+\frac{1}{4}\left\|\phi \otimes \phi^{*}-\tau\right\|_{L^{2}}^{2} .
$$

By [Bra90, Proposition 2.1], one has

$$
\begin{aligned}
\mathscr{Y} \mathscr{H}(A, \phi)=4\left\|F^{0,2}\right\|_{L^{2}}^{2}+ & 2\left\|\bar{\partial}_{A} \phi\right\|_{L^{2}}^{2}+\left\|\sqrt{-1} \Lambda F+\frac{1}{2} \phi \otimes \phi^{*}-\frac{\tau}{2}\right\|_{L^{2}}^{2} \\
& +\tau \int_{X} \sqrt{-1} \operatorname{tr} F \wedge \omega^{[n-1]}+\int_{X} \operatorname{tr} F \wedge F \wedge \omega^{[n-2]} .
\end{aligned}
$$

where $\omega^{[k]}:=\omega^{k} /(k !)$ and $\Lambda$ is the dual Lefschetz operator. 
16.2. Assume that $X$ is a projective curve, so that

$$
\operatorname{deg}(E)=\frac{\sqrt{-1}}{2 \pi} \operatorname{tr} F
$$

Then (16.1.2) immediately implies the Bogomolny-Prasad-Sommerfield inequality

$$
\mathscr{Y} \mathscr{H}(A, \phi) \geq 2 \pi \tau \operatorname{deg}(E),
$$

and the equality holds if and only if the vortex equation

$$
\begin{aligned}
F^{0,2} & =0 \\
\bar{\partial}_{A} \phi & =0 \\
-\sqrt{-1} \Lambda F & =\frac{1}{2}\left(\phi \otimes \phi^{*}-\tau \mathrm{id}_{E}\right)
\end{aligned}
$$

is satisfied. (16.2.3) and (16.2.4) are holomorphicities for $E$ and $\phi$, and (16.2.5) is a generalization of the constant central curvature equation.

16.3. By taking the trace of (16.2.5) and integrating over $X$, one obtains

$$
-2 \pi \operatorname{deg}(E)=\frac{1}{2}\|\phi\|_{L^{2}}^{2}-\frac{1}{2} \tau \operatorname{rank}(E) \operatorname{vol}(X)
$$

so that the condition

$$
\tau \geq \frac{4 \pi \operatorname{deg}(E)}{\operatorname{rank}(E) \operatorname{vol}(X)}
$$

is necessary for (16.2.5) to have a solution.

16.4. The slope of a holomorphic vector bundle $E$ is defined by

$$
\mu(E)=\frac{\operatorname{deg}(E)}{\operatorname{rank}(E)} .
$$

For a holomorphic section $\phi$ of $E$, we set

$$
\begin{aligned}
\hat{\mu}(E) & :=\sup \left\{\mu\left(E^{\prime}\right) \mid E^{\prime} \text { is a reflexive subsheaf of } E \text { of rank less than } E\right\}, \\
\mu_{M}(E) & :=\max \{\hat{\mu}(E), \mu(E)\}, \\
\mu_{m}(E, \phi) & :=\inf \left\{\frac{\operatorname{rank}(E) \mu(E)-\operatorname{rank}\left(E^{\prime}\right) \mu\left(E^{\prime}\right)}{\operatorname{rank}(E)-\operatorname{rank}\left(E^{\prime}\right)} \mid\right. \\
& \left.E^{\prime} \text { is a reflexive subsheaf of } E \text { such that rank } E^{\prime}<\operatorname{rank} E \text { and } \phi \in \Gamma\left(E^{\prime}\right)\right\} .
\end{aligned}
$$

A pair $(E, \phi)$ of a holomorphic vector bundle $E$ and its holomorphic section $\phi$ is said to be stable if

$$
\mu_{M}(E)<\mu_{m}(E, \phi) .
$$

Theorem 16.5 ([Bra91, Theorem 2.1.6]). Let $(E, \phi)$ be a pair of a holomorphic vector bundle and its holomorphic section. If there exists a Hermitian metric on E satisfying the vortex equation, then one has either of the following:

(i) $(E, \phi)$ is stable and satisfies

$$
\mu_{M}<\frac{\tau \operatorname{Vol}(X)}{4 \pi}<\mu_{m}(\phi) .
$$

(ii) $E$ has a direct sum decomposition $E=E_{\phi} \oplus E^{\prime}, \phi$ is an element of $H^{0}\left(E_{\phi}\right) \subset H^{0}(E)$, $\left(E_{\phi}, \phi\right)$ satisfies (i) above, and $E^{\prime}$ is the direct sum of stable vector bundles of slope $\tau \operatorname{Vol}(X) / 4 \pi$. 
Theorem 16.6 ([Bra91, Theorem 3.1.1]). Let $(E, \phi)$ be a stable pair of a holomorphic vector bundle and its holomorphic section. Then for any real number $\tau$ satisfying (16.5.1), there exists a Hermitian metric on E satisfying (16.2.5).

Bradlow proved these results not only for projective curves but also for compact Kähler manifolds.

16.7. Vortex equation (16.2.5) admits the following generalization, which also contains Hitchin's self-duality equation [Hit87] as a special case. Let $Q=\left(Q_{0}, Q_{1}, s, t\right)$ be a quiver and $M=\left(M_{a}\right)_{a \in Q_{1}}$ be a collection of vector bundles on $X$ labeled by $Q_{1}$. An $M$-twisted $Q$-sheaf on $X$ is a pair $R=\left(\left(E_{v}\right)_{v \in Q_{0}},\left(\phi_{a}\right)_{a \in Q_{1}}\right)$ of a collection $\left(E_{v}\right)_{v \in Q_{0}}$ of vector bundles labeled by $Q_{0}$ and a collection

$$
\left(\phi_{a}\right)_{a \in Q_{1}} \in \prod_{a \in Q_{1}} \operatorname{Hom}\left(E_{s(a)} \otimes M_{a}, E_{t(a)}\right)
$$

of morphisms labeled by $Q_{1}$.

Given a collection $\left(E_{v}\right)_{v \in Q_{0}}$ of holomorphic vector bundles on a Kähler manifold $X$, another collection $\left(M_{a}\right)_{a \in Q_{1}}$ of holomorphic vector bundles on $X$, a collection $\sigma=\left(\sigma_{v}\right)_{v \in Q_{0}}$ of positive real numbers, and a collection $\tau=\left(\tau_{v}\right)_{v \in Q_{0}}$ of real numbers, the equation

$$
\sigma_{v} \sqrt{-1} \Lambda F_{v}+\sum_{t(a)=v} \phi_{a} \circ \phi_{a}^{*}-\sum_{s(a)=v} \phi_{a}^{*} \circ \phi_{a}=\tau_{v} \operatorname{id}_{E_{v}}
$$

for Hermitian metrics on $\left(E_{v}\right)_{v \in Q_{0}}$ is called the $M$-twisted quiver $(\sigma, \tau)$-vortex equation.

The $(\sigma, \tau)$-degree and the $(\sigma, \tau)$-slope of an $M$-twisted $Q$-sheaf $R$ is defined by

$$
\begin{aligned}
\operatorname{deg}_{\sigma, \tau}(R) & =\sum_{v \in Q_{0}}\left(\sigma_{v} \operatorname{deg} E_{v}-\tau_{v} \operatorname{rank} E_{v}\right), \\
\mu_{\sigma, \tau}(R) & =\frac{\operatorname{deg}_{\sigma, \tau}(R)}{\sum_{v \in Q_{0}} \sigma_{v} \operatorname{rank} E_{v}} .
\end{aligned}
$$

A $Q$-sheaf is stable if one has $\mu_{\sigma, \tau}\left(R^{\prime}\right)<\mu_{\sigma, \tau}(R)$ for any proper subsheaf $R^{\prime}$. A $Q$-sheaf is polystale if it is the direct sum of stable $Q$-sheaf of the same slope.

Theorem 16.8 ([ÁCGP03, Theorem 3.1]). A $Q$-sheaf $R$ with $\operatorname{deg}_{\sigma, \tau}(R)=0$ admits a Hermitian metric satisfying the quiver vortex equation (16.7.2) if and only if $R$ is $(\sigma, \tau)$ polystable. This Hermitian metric is unique up to a multiplication by a positive constant for each stable summand.

Quasimaps to $\operatorname{Mat}(r, n) / / \mathrm{GL}_{r}$ corresponds to the case when the quiver $Q=(1 \rightarrow 2)$ consists of two vertices and one arrow between them, $M_{1}$ and $M_{2}$ are the structure sheaves, rank $E_{1}=r$, and $E_{2}$ is the trivial bundle of rank $n$.

16.9. Note that the map $V \rightarrow \operatorname{End}(V), \phi \mapsto \phi \otimes \phi^{*}$ appearing in (16.2.5) is the moment map for the natural action of the unitary group $U(V)$ on $V$. With this in mind, a generalization

$$
* F_{A}+\mu(\Phi)=\tau \operatorname{id}_{E}
$$

of the vortex equation (16.2.5) to the case where one has a Hamiltonian action of a compact group $G$ on a Kähler manifold $X$ is given in [MiR00, CGS00]. Here $A$ is a connection on a principal $G$-bundle on a curve $C, \Phi$ is a holomorphic section of $P \times_{G} X$, and $\mu: X \rightarrow \mathfrak{g}$ is the moment map. They are used to define invariants of a symplectic manifold with a Hamiltonian group action [CGS00, MiR03, CGMiRS02, 
which are closely related to the Gromov-Witten invariants of the symplectic quotient [GS05, Zil14, Woo15a, Woo15b, Woo15c]. CS06] use wall-crossing in vortex invariants to study quantum cohomology of monotone toric varieties with minimal Chern number greater than or equal to 2 .

16.10. Let $X$ be a Kähler manifold with a Hamiltonian action of a compact connected Lie group $G$. We assume that $X$ is either compact or equivariantly convex at infinity with a proper moment map. We fix an invariant inner product to identify $\mathfrak{g}^{\vee}$ with $\mathfrak{g}$, and write the moment map as $\mu: X \rightarrow \mathfrak{g}$.

An affine vortex is a pair $(A, u)$ of a connection $A$ on the principal bundle $P=\mathbb{C} \times G$ and a holomorphic section $u: C \rightarrow P \times{ }_{G} X$ satisfying the vortex equation

$$
* F_{A}+\mu(u)=0 \text {. }
$$

A gauged holomorphic map to $X$ with respect to the complex Lie group $G_{\mathbb{C}}$ acting on $X$ is a map to the quotient stack $\left[X / G_{\mathbb{C}}\right]$. In other words, a gauged holomorphic map from

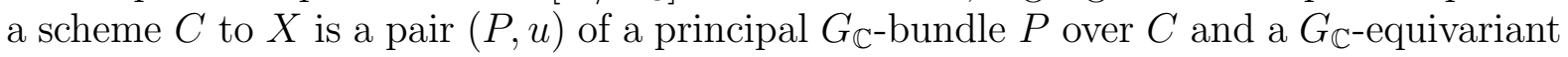
holomorphic map $u: P \rightarrow X$.

If the $G_{\mathbb{C}^{-} \text {action on }} X^{\mathrm{ss}}$ is free, then by [VW16, Theorem 1.1], there is a natural bijection between the set of affine $K$-vortices with target $X$ up to gauge equivalence and the set of pairs gauged holomorphic maps such that $u(\infty) \in X^{\mathrm{ss}}$. This is an open substack of the set of quasimaps such that $\infty$ is not contained in the base locus.

\section{REFERENCES}

[AB84] M. F. Atiyah and R. Bott, The moment map and equivariant cohomology, Topology 23 (1984), no. 1, 1-28. MR 721448

[ÁCGP03] Luis Álvarez-Cónsul and Oscar García-Prada, Hitchin-Kobayashi correspondence, quivers, and vortices, Comm. Math. Phys. 238 (2003), no. 1-2, 1-33. MR 1989667 (2005b:32027)

[BCFK05] Aaron Bertram, Ionut Ciocan-Fontanine, and Bumsig Kim, Two proofs of a conjecture of Hori and Vafa, Duke Math. J. 126 (2005), no. 1, 101-136. MR MR2110629

[BCFKvS98] Victor V. Batyrev, Ionut Ciocan-Fontanine, Bumsig Kim, and Duco van Straten, Conifold transitions and mirror symmetry for Calabi-Yau complete intersections in Grassmannians, Nuclear Phys. B 514 (1998), no. 3, 640-666. MR MR1619529 (99m:14074)

[BCFKvS00] _ Mirror symmetry and toric degenerations of partial flag manifolds, Acta Math. 184 (2000), no. 1, 1-39. MR MR1756568 (2001f:14077)

[BF97] K. Behrend and B. Fantechi, The intrinsic normal cone, Invent. Math. 128 (1997), no. 1, 45-88. MR MR1437495 (98e:14022)

[BM02] Victor V. Batyrev and Evgeny N. Materov, Toric residues and mirror symmetry, Mosc. Math. J. 2 (2002), no. 3, 435-475, Dedicated to Yuri I. Manin on the occasion of his 65th birthday. MR 1988969 (2005a:14070)

[BM03] _ Mixed toric residues and Calabi-Yau complete intersections, Calabi-Yau varieties and mirror symmetry (Toronto, ON, 2001), Fields Inst. Commun., vol. 38, Amer. Math. Soc., Providence, RI, 2003, pp. 3-26. MR 2019144 (2005b:14088)

[BMO11] Alexander Braverman, Davesh Maulik, and Andrei Okounkov, Quantum cohomology of the Springer resolution, Adv. Math. 227 (2011), no. 1, 421-458. MR 2782198 (2012h:14133)

[Bor05] Lev A. Borisov, Higher-Stanley-Reisner rings and toric residues, Compos. Math. 141 (2005), no. 1, 161-174. MR 2099774 (2005j:14074)

[Bra90] Steven B. Bradlow, Vortices in holomorphic line bundles over closed Kähler manifolds, Comm. Math. Phys. 135 (1990), no. 1, 1-17. MR 1086749 (92f:32053)

[Bra91] _ Special metrics and stability for holomorphic bundles with global sections, J. Differential Geom. 33 (1991), no. 1, 169-213. MR 1085139 (91m:32031)

[BV99] Michel Brion and Michèle Vergne, Arrangement of hyperplanes. I. Rational functions and Jeffrey-Kirwan residue, Ann. Sci. École Norm. Sup. (4) 32 (1999), no. 5, 715-741. MR 1710758 
[BvS95] Victor V. Batyrev and Duco van Straten, Generalized hypergeometric functions and rational curves on Calabi-Yau complete intersections in toric varieties, Comm. Math. Phys. 168 (1995), no. 3, 493-533. MR 1328251 (96g:32037)

[BZ15] Francesco Benini and Alberto Zaffaroni, A topologically twisted index for three-dimensional supersymmetric theories, J. High Energy Phys. (2015), no. 7, 127, front matter+75. MR 3383085

[CCFK15] Daewoong Cheong, Ionut Ciocan-Fontanine, and Bumsig Kim, Orbifold quasimap theory, Math. Ann. 363 (2015), no. 3-4, 777-816. MR 3412343

[CCP15] Cyril Closset, Stefano Cremonesi, and Daniel S. Park, The equivariant A-twist and gauged linear sigma models on the two-sphere, J. High Energy Phys. (2015), no. 6, 076, front matter+110. MR 3370259

[CdlOGP91] Philip Candelas, Xenia C. de la Ossa, Paul S. Green, and Linda Parkes, A pair of CalabiYau manifolds as an exactly soluble superconformal theory, Nuclear Phys. B 359 (1991), no. 1, 21-74. MR MR1115626 (93b:32029)

[CFK] Ionut Ciocan-Fontanine and Bumsig Kim, Quasimap theory, to appear in Proc. Internat. Congr. Mathematicians (Seoul, 2014).

[CFK14] Wall-crossing in genus zero quasimap theory and mirror maps, Algebr. Geom. 1 (2014), no. 4, 400-448. MR 3272909

[CFKM14] Ionut Ciocan-Fontanine, Bumsig Kim, and Davesh Maulik, Stable quasimaps to GIT quotients, J. Geom. Phys. 75 (2014), 17-47. MR 3126932

[CGMiRS02] Kai Cieliebak, A. Rita Gaio, Ignasi Mundet i Riera, and Dietmar A. Salamon, The symplectic vortex equations and invariants of Hamiltonian group actions, J. Symplectic Geom. 1 (2002), no. 3, 543-645. MR 1959059 (2004g:53098)

[CGS00] Kai Cieliebak, Ana Rita Gaio, and Dietmar A. Salamon, J-holomorphic curves, moment maps, and invariants of Hamiltonian group actions, Internat. Math. Res. Notices (2000), no. 16, 831-882. MR 1777853

[CK99] David A. Cox and Sheldon Katz, Mirror symmetry and algebraic geometry, Mathematical Surveys and Monographs, vol. 68, American Mathematical Society, Providence, RI, 1999. MR MR1677117 (2000d:14048)

[Cox96] David A. Cox, Toric residues, Ark. Mat. 34 (1996), no. 1, 73-96. MR 1396624

[CS06] Kai Cieliebak and Dietmar Salamon, Wall crossing for symplectic vortices and quantum cohomology, Math. Ann. 335 (2006), no. 1, 133-192. MR 2217687

[Don84] S. K. Donaldson, Nahm's equations and the classification of monopoles, Comm. Math. Phys. 96 (1984), no. 3, 387-407. MR 769355

[FJR18] Huijun Fan, Tyler J. Jarvis, and Yongbin Ruan, A mathematical theory of the gauged linear sigma model, Geom. Topol. 22 (2018), no. 1, 235-303. MR 3720344

[FR14] Michael Finkelberg and Leonid Rybnikov, Quantization of Drinfeld Zastava in type A, J. Eur. Math. Soc. (JEMS) 16 (2014), no. 2, 235-271. MR 3161283

[GH78] Phillip Griffiths and Joseph Harris, Principles of algebraic geometry, Wiley-Interscience [John Wiley \& Sons], New York, 1978, Pure and Applied Mathematics. MR 507725 (80b:14001)

[Giv95a] A. B. Givental', Homological geometry. I. Projective hypersurfaces, Selecta Math. (N.S.) 1 (1995), no. 2, 325-345. MR MR1354600 (97c:14052)

[Giv95b] Alexander B. Givental, Homological geometry and mirror symmetry, Proceedings of the International Congress of Mathematicians, Vol. 1, 2 (Zürich, 1994) (Basel), Birkhäuser, 1995, pp. 472-480. MR MR1403947 (97j:58013)

[Giv96] Alexander Givental, Equivariant Gromov-Witten invariants, Internat. Math. Res. Notices (1996), no. 13, 613-663. MR MR1408320 (97e:14015)

[Giv98] A mirror theorem for toric complete intersections, Topological field theory, primitive forms and related topics (Kyoto, 1996), Progr. Math., vol. 160, Birkhäuser Boston, Boston, MA, 1998, pp. 141-175. MR MR1653024 (2000a:14063)

[GRTV13] V. Gorbounov, R. Rimányi, V. Tarasov, and A. Varchenko, Quantum cohomology of the cotangent bundle of a flag variety as a Yangian Bethe algebra, J. Geom. Phys. 74 (2013), 56-86. MR 3118573

[GS05] Ana Rita Pires Gaio and Dietmar A. Salamon, Gromov-Witten invariants of symplectic quotients and adiabatic limits, J. Symplectic Geom. 3 (2005), no. 1, 55-159. MR 2198773 
[GW13] Eduardo Gonzalez and Chris Woodward, Gauged Gromov-Witten theory for small spheres, Math. Z. 273 (2013), no. 1-2, 485-514. MR 3010172

[Hit82] N. J. Hitchin, Monopoles and geodesics, Comm. Math. Phys. 83 (1982), no. 4, 579-602. MR 649818

[Hit83] $\quad$ On the construction of monopoles, Comm. Math. Phys. 89 (1983), no. 2, 145-190. MR 709461

[Hit87] _ The self-duality equations on a Riemann surface, Proc. London Math. Soc. (3) 55 (1987), no. 1, 59-126. MR 887284 (89a:32021)

[HV] Kentaro Hori and Cumrun Vafa, Mirror symmetry, hep-th/0002222.

[Int91] Kenneth Intriligator, Fusion residues, Modern Phys. Lett. A 6 (1991), no. 38, 3543-3556. MR 1138873 (92k:81180)

[Iri09] Hiroshi Iritani, An integral structure in quantum cohomology and mirror symmetry for toric orbifolds, Adv. Math. 222 (2009), no. 3, 1016-1079. MR 2553377 (2010j:53182)

[Iri11] _ Quantum cohomology and periods, Ann. Inst. Fourier (Grenoble) 61 (2011), no. 7, 2909-2958. MR 3112512

[Jar98a] Stuart Jarvis, Construction of Euclidean monopoles, Proc. London Math. Soc. (3) 77 (1998), no. 1, 193-214. MR 1625471

[Jar98b] ㄴ Euclidean monopoles and rational maps, Proc. London Math. Soc. (3) 77 (1998), no. 1, 170-192. MR 1625475

[Kar05] Kalle Karu, Toric residue mirror conjecture for Calabi-Yau complete intersections, J. Algebraic Geom. 14 (2005), no. 4, 741-760. MR 2147350 (2006c:14063)

[KM10] Yukiko Konishi and Satoshi Minabe, Local B-model and mixed Hodge structure, Adv. Theor. Math. Phys. 14 (2010), no. 4, 1089-1145. MR 2821394 (2012h:14106)

[Mar] S. Martin, Symplectic quotients by a nonabelian group and by its maximal torus, math.SG/0001002.

[Mav00] Anvar R. Mavlyutov, Semiample hypersurfaces in toric varieties, Duke Math. J. 101 (2000), no. 1, 85-116. MR 1733735

[MiR00] Ignasi Mundet i Riera, A Hitchin-Kobayashi correspondence for Kähler fibrations, J. Reine Angew. Math. 528 (2000), 41-80. MR 1801657

[MiR03] _ Hamiltonian Gromov-Witten invariants, Topology 42 (2003), no. 3, 525-553. MR 1953239 (2004d:53106)

[MiRT09] I. Mundet i Riera and G. Tian, A compactification of the moduli space of twisted holomorphic maps, Adv. Math. 222 (2009), no. 4, 1117-1196. MR 2554933

[Miu13] Makoto Miura, Hibi toric varieties and mirror symmetry, Ph.D. thesis, The University of Tokyo, 2013.

[MO19] Davesh Maulik and Andrei Okounkov, Quantum groups and quantum cohomology, Astérisque (2019), no. 408, ix+209. MR 3951025

[MP95] David R. Morrison and M. Ronen Plesser, Summing the instantons: quantum cohomology and mirror symmetry in toric varieties, Nuclear Phys. B 440 (1995), no. 1-2, 279-354. MR 1336089 (96f:32036)

[Muk92] Shigeru Mukai, Polarized K3 surfaces of genus 18 and 20, Complex projective geometry (Trieste, 1989/Bergen, 1989), London Math. Soc. Lecture Note Ser., vol. 179, Cambridge Univ. Press, Cambridge, 1992, pp. 264-276. MR 1201388 (94a:14039)

[Nah82] W. Nahm, The construction of all self-dual multimonopoles by the ADHM method, Monopoles in quantum field theory (Trieste, 1981), World Sci. Publishing, Singapore, 1982, pp. 87-94. MR 766754

[Nak12] Hiraku Nakajima, Handsaw quiver varieties and finite $W$-algebras, Mosc. Math. J. 12 (2012), no. 3, 633-666, 669-670. MR 3024827

[NS09] Nikita A. Nekrasov and Samson L. Shatashvili, Supersymmetric vacua and Bethe ansatz, Nuclear Phys. B Proc. Suppl. 192/193 (2009), 91-112. MR 2570974 (2011i:81217)

[Oko17] Andrei Okounkov, Lectures on K-theoretic computations in enumerative geometry, Geometry of moduli spaces and representation theory, IAS/Park City Math. Ser., vol. 24, Amer. Math. Soc., Providence, RI, 2017, pp. 251-380. MR 3752463

[ST97] Bernd Siebert and Gang Tian, On quantum cohomology rings of Fano manifolds and a formula of Vafa and Intriligator, Asian J. Math. 1 (1997), no. 4, 679-695. MR 1621570 (99d:14060) 
[Sta12] Matthias Staudacher, Review of AdS/CFT integrability, Chapter III.1: Bethe ansätze and the R-matrix formalism, Lett. Math. Phys. 99 (2012), no. 1-3, 191-208. MR 2886419

[SV04] András Szenes and Michèle Vergne, Toric reduction and a conjecture of Batyrev and Materov, Invent. Math. 158 (2004), no. 3, 453-495. MR 2104791 (2005i:14065)

[SV06] Mixed toric residues and tropical degenerations, Topology 45 (2006), no. 3, 567599. MR 2218757

[Toë14] Bertrand Toën, Derived algebraic geometry, EMS Surv. Math. Sci. 1 (2014), no. 2, $153-240$. MR 3285853

[TX] Gang Tian and Guangbo Xu, Analysis of gauged Witten equation, arXiv:1405.6352.

[Vaf91] Cumrun Vafa, Topological Landau-Ginzburg models, Modern Phys. Lett. A 6 (1991), no. 4, 337-346. MR 1093562 (92f:81193)

[VW16] S. Venugopalan and C. Woodward, Classification of affine vortices, Duke Math. J. 165 (2016), no. 9, 1695-1751. MR 3513572

[Wit93] Edward Witten, Phases of $N=2$ theories in two dimensions, Nuclear Phys. B 403 (1993), no. 1-2, 159-222. MR MR1232617 (95a:81261)

[Wit95] The Verlinde algebra and the cohomology of the Grassmannian, Geometry, topology, \& physics, Conf. Proc. Lecture Notes Geom. Topology, IV, Int. Press, Cambridge, MA, 1995, pp. 357-422. MR 1358625 (98c:58016)

[Woo15a] Chris T. Woodward, Quantum Kirwan morphism and Gromov-Witten invariants of quotients I, Transform. Groups 20 (2015), no. 2, 507-556. MR 3348566

[Woo15b] _ Quantum Kirwan morphism and Gromov-Witten invariants of quotients II, Transform. Groups 20 (2015), no. 3, 881-920. MR 3376153

[Woo15c] Quantum Kirwan morphism and Gromov-Witten invariants of quotients III, Transform. Groups 20 (2015), no. 4, 1155-1193. MR 3416443

[Zil14] Fabian Ziltener, A quantum Kirwan map: bubbling and Fredholm theory for symplectic vortices over the plane, Mem. Amer. Math. Soc. 230 (2014), no. 1082, vi+129. MR 3221852

Korea Institute for Advanced Study, 85 Hoegi-ro Dondaemun-gu Seoul 02455, RepubLIC OF KOREA

E-mail address: bumsig@kias.re.kr

Korea Institute for Advanced Study, 85 Hoegi-ro Dondaemun-gu Seoul 02455, RepubLIC OF KOREA

E-mail address: batistuta@kaist.ac.kr

Graduate School of Mathematical Sciences, The University of Tokyo, 3-8-1 Komaba MEGURO-KU TOKYO 153-8914 JAPAN.

E-mail address: kazushi@ms.u-tokyo.ac.jp

Kavli IPMU (WPI), UTIAS, University of Tokyo Kashiwa, Chiba 277-8583, Japan

E-mail address: yyyyosida@gmail.com 